\title{
Microsismi da piccoli hacini chiusi, da mari interni, da Oceani
}

\author{
P. CAIOI - M. MIgaNi
}

Ricevuto il 10 Ottobre 1971

Riassonto, - $\mathrm{E}$ il compendio di una lunga serie di ricerche sui microsismi e sulla loro origine.

Il noto ondoso ì la causa immediata doi microsismi, come efot to di trasferimento sli energia sul fondo. Il ritmo dei microsismi fondamentali (quelli legati a sistemi d'onde significative) è legato all'estensione lel bacino itlrieo e alla sua profonditì.

Por la prima volta, si portano numerosi esempi di microsismi, provoeati sul fondo di un minuscolo lago (il lago di Pievo di Cadore), come conseguenza elell'interazione fra rapide variazioni dolla pressione atmosforica, in transito sul lago, o le acque sottostanti; i periodi prevalenti si aggirano sugli $8 / 10$ di sec, ma possono variare fra i 4/10 lli sec e i 5 sec.

I periodi fondamentali di microsismi da mari interni, variano al variare delle caratteristiche della zona ili mare, da cui prentono origine. Cosi nel nolfo ali Trieste, i microsismi fondamentali presentano periodi dell'ordino li 2,5-3 sec: essi vengono fortomente amplificati nella loro propagazione attraverso la Val Padana. I microsismi fondamentali del Mar Jigure hamno periodi dellordine di $5 \pm 1$ sec: essi vengono sensibilmente smorzati dalla catena appenninica, mentre si propagano verso lalta Val Paclana, attraverso la "finestra " fra gli Appennini e le Alpi.

Dalla zona ad oceidente delle isolo britanniche, originano microsismi fontamentali con periodi di 8 sec ca: essi si propagano allo massimo distanze e la loro attenuazione in corrispondenza dei sistemi montuosi, non è accentuata. Al largo delle coste scandinave, prendono origine microsisni fondamentali con periodi dell'ordine di $5.6 \mathrm{sec}$.

I microsimi fonclamentali di massinta ampiezza naseono da fenomeni di risonanza cinematica fra perturbazioni microbarografiche $\theta$ acque sottostanti.

Localmento, in stazioni sismiche funzionanti sulle rive del maro, possono originare, nello stesso tempo, microsismi dai più svariati periodi: da 'quelli di pochi decimi li seconclo o di qualche secondo (dovuti a sistemi d'onle sporadici, temporanei accidentali), fino a quelli associati ai sistemi d'onle significative: sono questi ultimi i microsismi fonclamentali, animati da notovole onergia, $\theta$ che si propagano alle massime distanze. 
Poiche i microsismi non subiscono, nella loro propagazione, dispersione apprezzabile, dal periodo dei mierosismi fondamentali si puo risalire alla loro zona oricrine.

SUMmary. - This is the sum of a long series of research work on mirroseisms and their origin.

The wavy movement is the immediate cause of microseisms, as a result of the trangfer of energy on the ground. The rlythm of fundamental microseisms (depending on significant wave systems) is bound up with the extension of tlie liydric basin and its lepth.

For the first time numerous examples are given of microseisms ori. girating on the bottom of a very small lake (the Lake of Pieve di Cadore) as a conseruence of the interaction between rapid variations of the atmospleric pressure-passing over the lake and the water below; the prevailing periods are about $0,8 \mathrm{sec}$, but they nay vary between 0,4 and $5 \mathrm{sec}$.

The fundamental periods of mieroseisms leriving from inland seas vary with the properties of the sea area where they take their orjgin. In the Gulf of Trieste, for instance, the fundamental microseisms have periods in the order of $2,5-3$ sec.: they are strongiy amplified as they spreal over the Po Valley. The funclamental microseisms of the Ligurian Sea have periods in the order of $5 \pm \mathrm{l}$ sec and are sizenbly reluced by the Appenine monntain range as they spread toward the higher Po Valley passing through the "window" between the Appenines and the Alps.

The western area of the British Isles is the origin of fundamental mieroseisms with periods around $8 \mathrm{sec}$. They spread to maximum distances and their attenuation due to the mountain systems is not considerable. Fundamental microseisms with periols in the order of $5-6$ sec take their origin off the Scandinavian coast.

The fundamental microseisms of maximum amplitudes derive from plenomena of cinematic resonanee from mierobarograplic perturbations on the unflerlying water.

Microseisms of a wide range of periols may originate locally, at the same time, in seismie stations on the sea shores. They have periods of fow tenthis of seconds or of some seconds (due to sporalic, temporary, easual wave systems), and there are microsoisms associated to the systems of significant waves. The latter are fundamental, driven by considerabie energies and spread to maximum distances.

Since the spreading of microseisms is not subject to appreciable dispersions, the period of fundamental mieroseisms allows to letect their area of origin.

\section{Microsismi dat، lago di Pleve di Cadore.}

Allo scopo di approfondire lo studio delle caratteristiche geodinamiche della diga di Pieve di Cadore, si è ritenuto opportuno sistemare una stazione sismica nel corpo della diga. 
Essa consiste in una terna di sismografi elettro-magnetici "Girlanda $n$, analoga a quella funzionante nello scantinato della cabina comandi, costruita a quota coronamento, presso la diga stessa.

La nuova stazione sismica, che ha iniziato il suo funzionamento ai primi di Juglio 1970, ha gia consentito la precisazione di particolarita, atte a delineare il comportamento dello sbarramento in determinate situazioni: sn esse sarà riferito in seguito.

$\mathrm{Di}$ eccezionale interesse si rivelarono le registrazioni, ottennte in diga (fig. 1), durante il passaggio di temporali o semplici groppi o

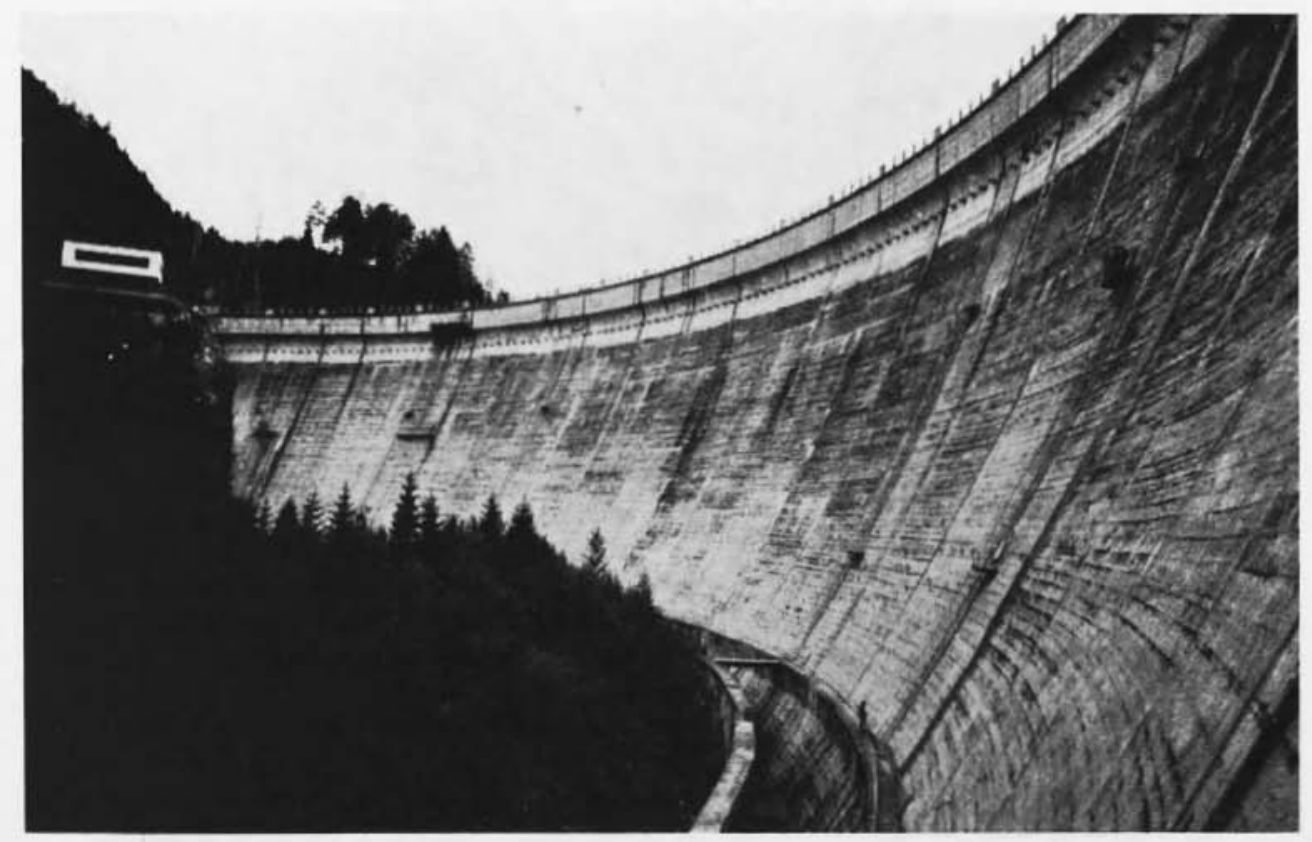

Fig. 1 - Diga di Pieve di Cadore. La stazione sismica, che ha registrato i microsismi provocati dal transito di disturbi microbarici, di cui alle figg. 6, 9-18 è sistomata (con funzioni di controllo geodinamico dol manufatto) in un cunicolo sfociante nel ballatoio, in alto, a destra nella fotografia. Analoga stazione sismica funziona negli seantinati della cabina comandi, di fianco alla diga (in alto, a sinistra, nella fotografia).

Fig. 1 - Dam of Pieve di Cadore. The seismic station which recorded the microseisms caused by passing microbaric perturbations (see fig. 6, 9-18) is lodged, with functions of geodynamic control of the structure, in a small tunnel connected with the open gallery appearing on the upper right side of the photo. An equal seismic station is operating in the basement of the control box at the side of the dam (on the upper left side of the photo). 
nuclei ciclonici. Già il 15 Luglio 1970 , il passaggio di una depressione - procedente da Ovest a Est - provocava la registrazione di microsismi di breve periodo ( $8 / 10 \mathrm{sec}$, in media) e notevole ampiezza. Su di essi ha già riferito uno di noi $(3,4)$. Qui intendiamo soffermarci più particolareggiatamente su quella tempesta microsismica e su tutta una serie di altre registrazioni di microsismi, collegati ad altri temporali in transito o a semplici passaggi di perturbazioni microbariche, in fase positiva o no, dotate di piccolissima energia.

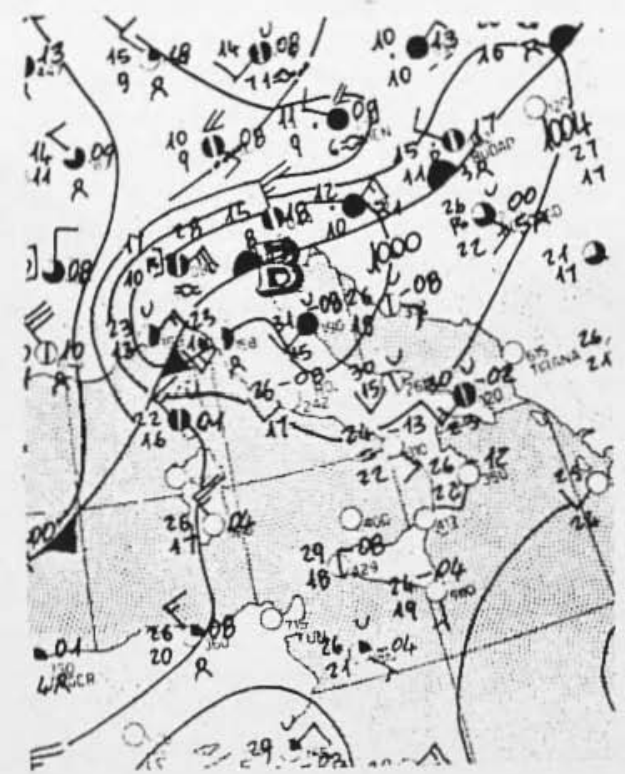

Fig. 2 - Posizione del centro della depressione del 15 Luglio 1970, quando, alle ore 18 (T.M.G.) - dopo aver provocato i microsismi passando sul lago di Piove di Cadore (fig. 5) - sta attraversando il golfo di Venozia, determi. nando una nuova, più ampia tempesta microsismica (figg. 6, 19.25).

Fig. 2 - Position of the centre of the depression of July 15, 1970 which at $6^{\text {h }}$ p.m. (Mean Solar Time), after having eaused the microseisms on passing over the Pjeve di Cadore Lake (fig. 5), is erossing the Gulf of Venice where it gives rise to another, ampler microseismic storm (figures 6, 19-25).

La fig. 2 rappresenta un momento della situazione meteorologica del 15 Luglio 1970, quanilo il ciclone - dopo essere transitato sul lago di Pieve di Cadore e ivi provocato la registrazione di minutissimi, ampli microsismi - di cui alla fig. 6 - ha raggiunto, nella sua corsa verso ESE, l'alto Adriatico e causato l'insorgere di microsismi, 
propri del golfo di Trieste, chiaramente registrati anche a Pieve di Cadore, circa 3 ore dopo la registrazione rlei caratteristici microsismi del lago (fig. 6). Di questa seconda, ben più energica tempesta microsismica, associata allo stesso ciclone, sarà detto piì estesamente al n. 2 .

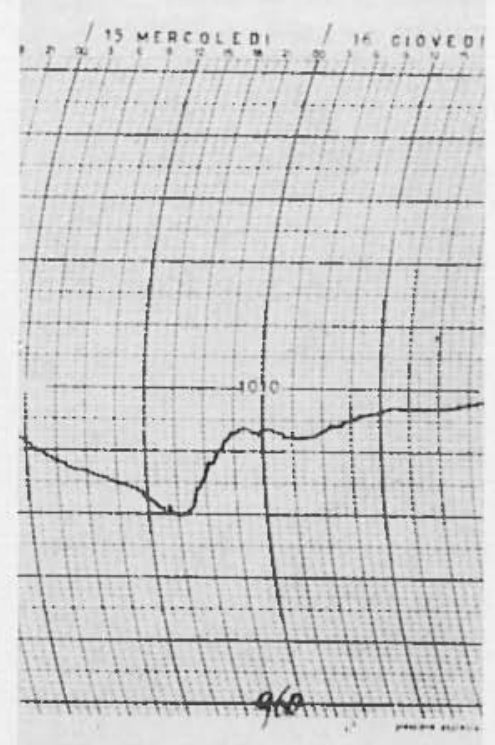

Fig. 3 - Andamento della pressione atmosferica a S. Angelo (Treviso), dopo il transito del ciclone del 15. VII.1970 su Pieve di Calore (T.M.G.).

Fig. 3 - Development of the atmospheric pressure at Sant'Angelo (Treviso) after the passage of the cyclone of July 15, 1970 over Pieve di Cadore (Mean Solar Time).

Le figg. 3, 4 mostrano l'andamento della pressione a $\mathrm{S}$. Angelo (Treviso), e a Trieste, in concomitanza con il passaggio della depressione. it in corrispondenza delle rapide variazioni barografiche, che accompagnano la ripresa in fase positiva della pressione atmosferica, che insorgono i microsismi nel lago di Pieve di Cadore: primo esempio di cospicui microsismi, associati a rapide variazioni della pressione in fase positiva, registrati presso un minuscolo lago (fig. 5).

Naturalmente, il fenomeno si è ripetuto. Una depressione ciclonica di intensità molto minore di quella del 15 Luglio, si presentò 
sul Vencto il 16 Settembre 1970 , seguenilo l'abituale tragitto da Ovest arl Est. La fig. 7 riproluce la situazione barografica alle ore 18 (Gr.).

Vella fig. 8 è riportato l'aniamento della pressione atmosferica, registrata in quell'oceasione arl Arta Terme (a pochi chilometri da 'Tolmezzo).

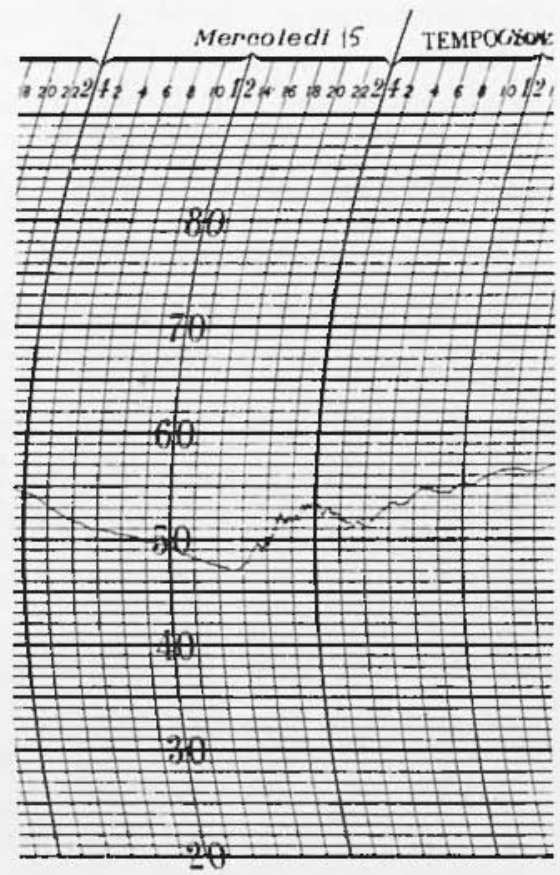

Fir. 4 - Andamento della pressione atmosferica a Trieste, durante il transito della depressione clel 15.VII.1970 (il tempo è quello solare merlio). si notino le rapidissime variazioni della pressione in fase positiva.

Fig. 4 - Development of atmospheric pressure at Trieste during the passage of the depression of July 15, 1970 (Mean Solar Time). The very rapirl variations of pressure in the positive phase are to he noted.

Transitando sul lago di Pieve di Cadore, quella perturbazione atmosferiea provocó microsismi, analoghi a quelli ottennti il 15 Luglio, sebbene di minore ampiezza, attesa la minore energia associata alla perturbazioue (fig. 9). Anche questa volta, ragginnto il golfo di Trieste, il ciclone đeterminò l'insorgere dei microsismi propri del golfo, visibili sia « Padova, a Pieve di Cadore, a Ta Maina (figg. 27, 9, 26) ecc, sebbene di ampiezza nettamente inferiore a quella registrata il 15 Iuglio, per il motivo più volte accennato. 
Per provocare microsismi nel piccolo lago di Pieve di Cadore non è però necessario il passaggio di perturbazioni atmosferiche di notevole energia, come quelle sopra eitate. Anche perturbazioni secondarie, di energia molto ridotta, sono capaci di causare la registrazione di apprezzabili microsismi; come p.es. si è verificato il 25 Luglio, il 3 Agosto, il 7 Agosto 1970 ece. durante il passaggio di perturbazioni barografiche di lieve entità.

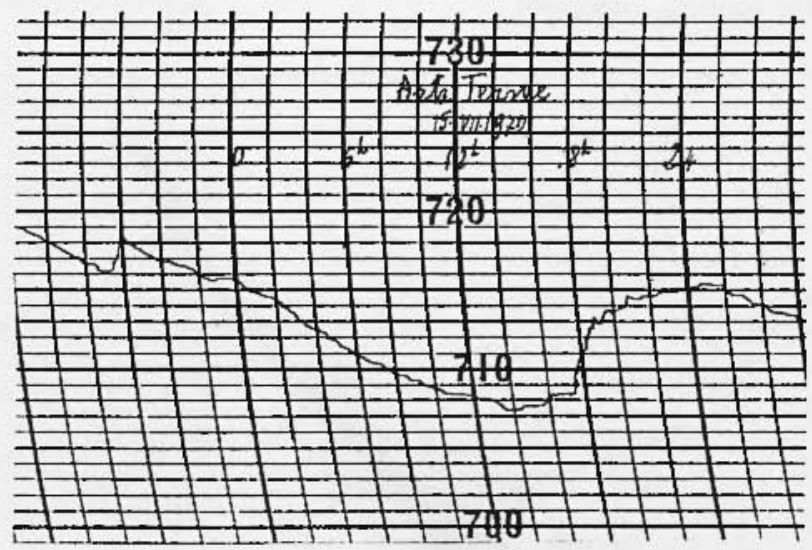

Fig. 4 bis - Andamento della pressione atmosferica ad Arta Terme, il 15. VII 1970 (ora solare). La depressione si è presentata, quel giorno, nettamente più profonda di quella del 16.IX.1970, anche se presenta con essa qualche analogia. Ja maggiore attività barografica del 15.VII.1970 si ò riflessa in una maggiore ampiezza dei microsismi, sia a Pieve di C. sia nel golfo di Trieste, di quella registrata il 16.IX.1970.

Fig. 4 bis. - Development of atmospheric pressure at Arta Terme, on July 15, 1970 (Mean Solar Time). On that day the depression appeared to be clearly rleeper than that of September 16, 1970, even if in some aspects it is similar to it. The higher barographic activity of July 15, 1970 was reflected in a major amplitude of mieroseisms, both in Pieve di Cadore and in Trieste, compared with that recorded on September 16, 1970.

A questo punto, risultava evidente che l'interazione aria-acqua, eapace di originare microsismi dal lago, poteva essere provocata da fluttuazioni di energia, in movimento sull'acqua, estremamente piccole.

Per registrare tali fluttuazioni di pressione, si rendeva necessario l'uso di un microbarografo.

Nell'Ottobre 1970 fu sistemato, nella cabina-comandi della diga di Pieve di Cadore, un microbarografo "Askania ", capace di 
registrare variazioni di pressione di una frazione di un Torricelli (1. Torr $=1 \mathrm{~mm} \mathrm{Hg}$ ).

Le registrazioni di questo strumento confermarono in pieno le nostre previsioni: il passagrio di lievi disturbi microbarici, in fase positiva o no, dissociati da groppi o temporali, in condizioni di tempo bello - e registrati dal solo microbarografo — è cajace di provocare microsismi, chiaramente registrati dalla stazione sismica, sistemata nell'interno della diga e - in minor misura - anche dalla stazione sismicá nella cabina comandi.

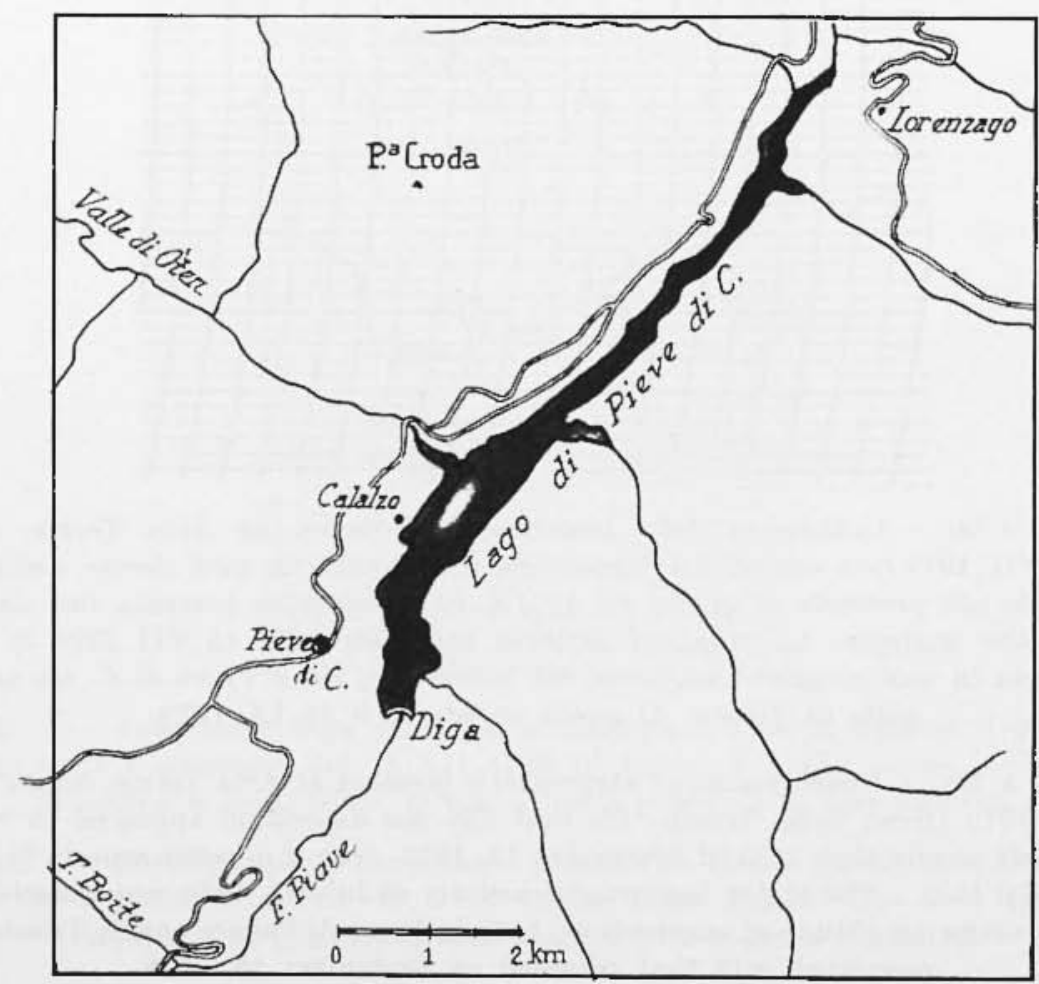

Fig. 5 - 11 lago di Pieve di Cadlore, formato dallo sbarramento del Piave in località Sottocustello, è lungo ... a pieno carico ...- circa $9 \mathrm{~km}$. Presenta nna profonlitì massima di cirra $100 \mathrm{~m}$, in corrispondenza di un piccolo settore (quello di destra) della diga; nel rimunenie contatto con la diga, l'acqua raggiunge la profondità massima di $60 \mathrm{~m}$ ca.

Fig. 5 - The Lake of Pieve di Cadore, formed by blocking the Piave at Sottocastello, is about $9 \mathrm{kms}$ long when entirely fillecl. Its maximum depth is about $100 \mathrm{~ms}$ which corresponds to a small section (at the right) of the diun.

The lake depth at the rest of the dam is not over $60 \mathrm{~ms}$. 
P. Calot - M. Mtiani

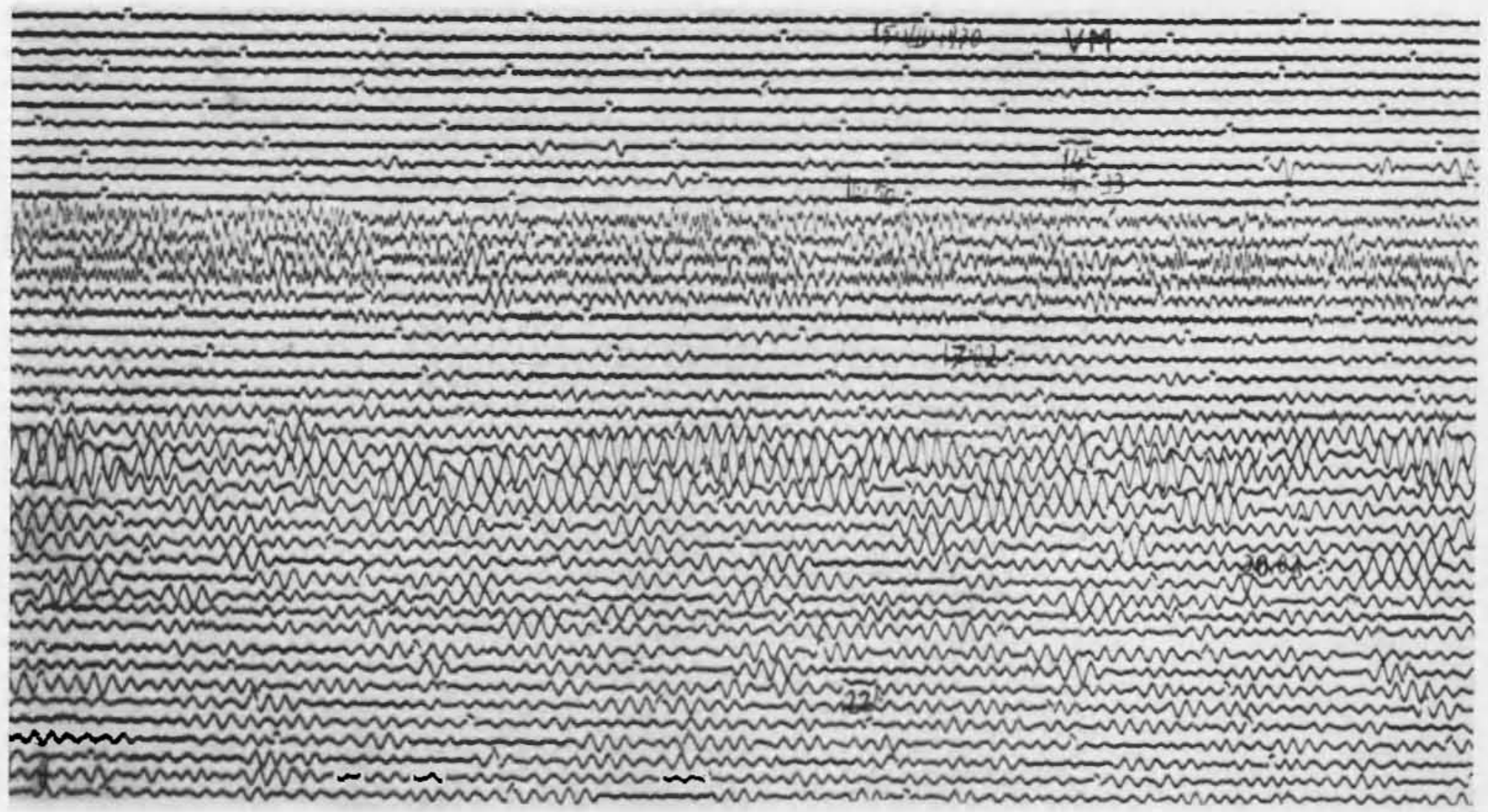

Fig. 6a.

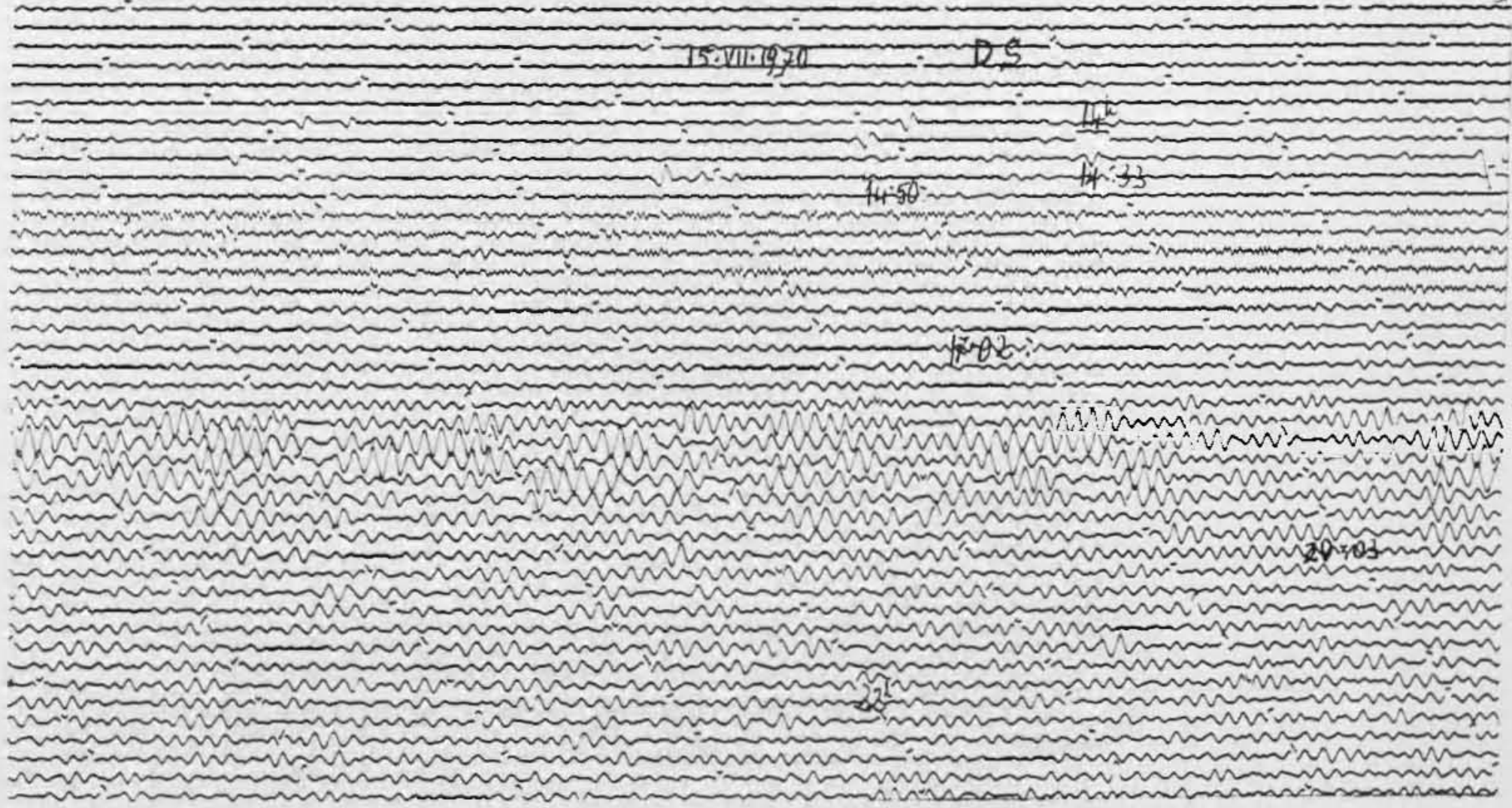

Fig. 6b.

Fig. 6 - Registrazione di mierosismi ottenuti a Pieve di Cadore, sul lago omonimo, associati al transito del eiclone del 15. VII. 1970. Si osservano due fasi distinte: una dalla $14^{\mathrm{n}} \mathbf{4}^{\mathrm{m}}$ ea (T.M.G.) alla $16^{\mathrm{h}} \mathrm{5}^{\mathrm{m}}$ ca; l'altra dalle $17^{\mathrm{m}} 30^{\mathrm{m}}$ ea in poi. La prima fase $\rightarrow$ la più interessante in quanto costituisce il fatto nuovo - o quella formata dai microsismi, causati dal transito del eentro del ciclone diret. tamente sul lago, come conseguenza dell'interazione fra la turbolenza della pressione atmosferica, in fase positiva, e le acque del lago sottostante: di qui la formazione di mierosismi rapidi, di periodo generalmente inferiore al secondo. La seconda fase è invece legata al transito del centro dello stesso ciclone sul golfo di Venezia. Come presso le altre stazioni d'osservazione (tigg. 19-25), anche a Pieve di Cadore $\mathrm{i}$ massimi per $\mathrm{i}$ microsismi provenienti dall'Allriatico si presentano dalle $18^{\mathrm{n}} 30^{\mathrm{m}}$ circa in poi, quando il ciclone transita sul golfo di Trieste, ad una distanza da Pieve di Cadore di cirea $125 \mathrm{~km}$. Il periodo medio dei microsismi, per questa $2^{\mathrm{a}}$ tempesta (seconda, per Pieve) è come per le altre stazioni sismiche, di circa $2^{\mathrm{s}, 6}$. Sulla componente IV $V$ (monte-valle), normale alla superficie della diga, per motivi evidenti, l'agitazione microsismiea loeale è di ampiezza nettamente maggiore che sulla comp. DS (dlestra-sinistra), tangenziale alla superficie della diga.

Fig. 6 - Recordings of microseismic movements, obtained at Pieve di Cadore, on the lake of the same name, associated with the Passage of the cyclone of July 15, 1970. Two separate phases may be observed, the first from $2^{\mathrm{h}} 40^{\mathrm{m}} \mathrm{p} \cdot \mathrm{m}$. (Mean Solar Time) to $4^{\mathrm{h}} 50^{\mathrm{m}}$ p.rn. approximately, the second from $5^{\mathrm{h}} 3 \mathrm{~g}^{\mathrm{m}} \mathrm{p} \cdot \mathrm{m}$. on ward. The first phase, which is of more interest as it contains the new fact, is formed by microseisms provoked by the passage of the cyclone centre directly over the lake as a consequence of the interaction between the turbulance of the atmospheric pressure in the positive phase, and the underlying lake water which gives rise to rapid microseisms with periods generally lower than one second. The second phase is linked, however, to the passage of the centre of the same cyclone over the Gulf of Venice. As is the case also with the other observation stations (fig. 19-25), the maxima of microseisms coming from the Adriatic region occur at Pieve di Cadore beginning at about $6{ }^{6} 30^{\mathrm{m}}$ p.rn., when the eyclone passes over the Gulf of Trieste, at a distance of some $125 \mathrm{~km}$ from Pieve di Cadore. The mean period microseisms of this seconl storm (the second for Pieve) is about 2 s, 6 , as with the other seismic stations. For obvious reasons the microseismic local agitation on the hill-valley component, normal to the dam surface, has a sizeably larger amplitude as compared with the right-left component, tangential to the lam surface. 
Gli esempi ottenuti sono numerosissimi. Ci limitiamo a riportaune solo alemi (figg. 10-18).

Linteresse di queste registrazioni i veramente notevole: non esitiamo a refinirlo ecezionale. Por la prima volta, chiare registrazioni di mierosismi vengrono ottenute ai margini di un minuscolo bacino idrico, e - nel rontempo - ne viene isolata la sausa.

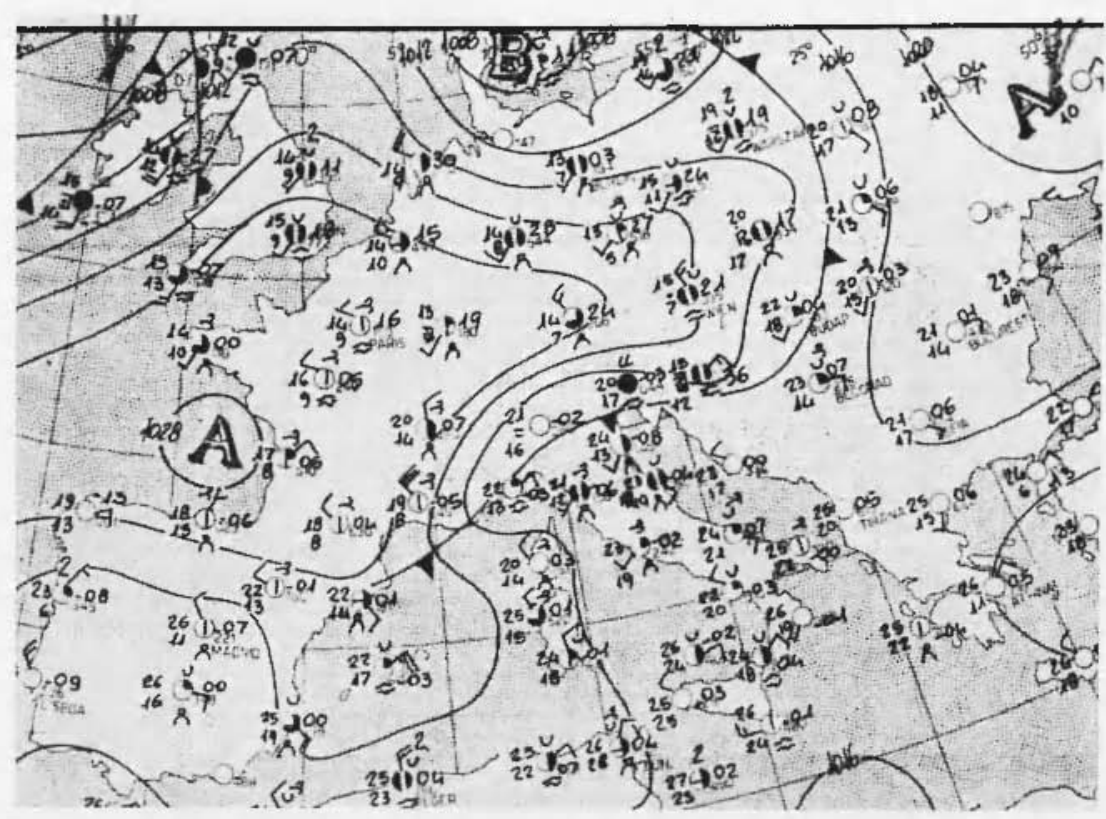

Fig. 7 - Situazione neteorologica del 16.1X.1970, alle ore 18 (T.M.G.), quando il fronte freddo ha ragginnto il golfo di Trieste, dopo esiere transitato

sul lago cli P’ieve di Cadore, cansandovi i microsismi di eni alla fig. 9.

Firr. 7 - The meteorological situation on soptenber 16 , 1970 , at $6^{1 / 1}$ p.m. (Mean Solar Time) whon the cold front reached the Giulf of Trieste after having passerl over the Like of l'ieve di Cadore, causing there the mirroseims showl on fig. 9.

Si noti che i microsismi iniziano e terminano con l'iniziare e il terminare delle lievi fluttumioni della pressione che, interagendo con le acque del lago, ne provocano l'insorgere (*). Si possono cosi avere

(*) Va sottolineato che da meta Dicembre a metì Harzo circa, malgrado il passaggrio di notevoli perturbazioni atmosferiche, non si hanno registrazioni di microsismi presso la iliga di Pieve di Cadore. La spiegazione è semplice. Durante quei mesi, infatti, il lago gela, interrompendo così ogni 
microsismi per la durata di una mezzora, od anche meno, pari al tempo di transito dei lievi imjulsi microbariei, da cui traggono origine. Se si pensa alle tempeste microsismiche oceaniche, che possono durare kettimane, associate a cicloni animati da energie poderose, che sconvolgono il mare e l'aria, si vede l'interesse di microsismi originanti in un piccolo bacino, jrovocati da una causa ben individuata, esauriente la sua azione in poche diecine di minuti: è l'origine dei microsismi - almeno di questo particolare tijo di microsismi - che viene precisata.

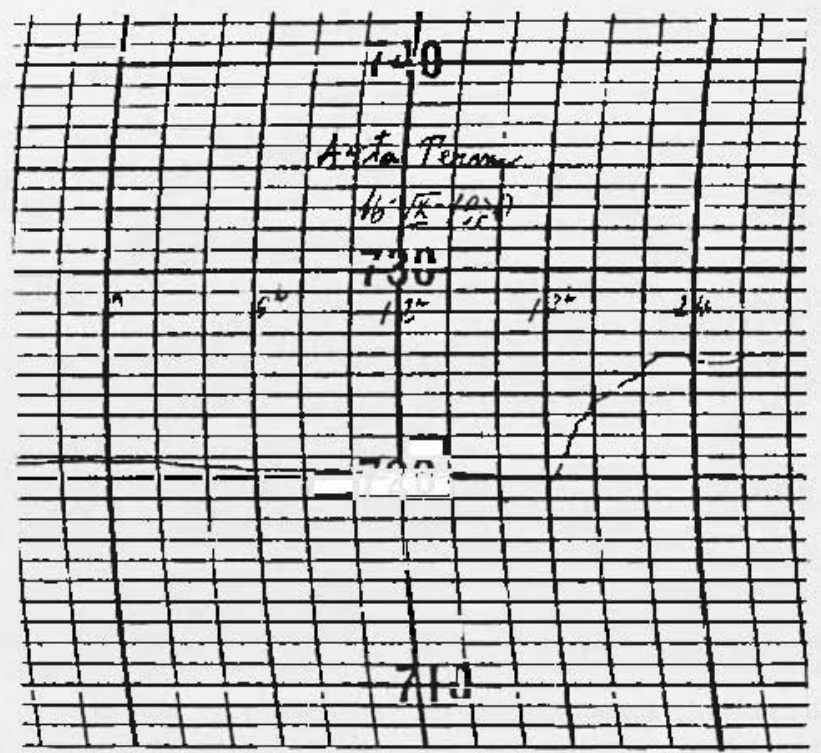

Fig. 8 - Andamento della pressione ad Arta Terme (nei pressi di Tolmezzo), durante il passaggio della lieve depressione del 16.IX.1970 (ora solare). Si noti il salto di pressione (aceompagnato da rapire fluttuazioni) a partire dalle $18^{\mathrm{h}}$, che presenta qualche analogia con quello osservato, presso la stessa stazione meteorologica, il 15 . VII. 1970 (fig. 4 bis).

Fig. 8 - Development of pressure at Arta Terme (near Tolmezzo) during the passage of the light depression of September 16, 1970 (Solar Time). The sudden pressure increase, accompanied by rapid fluctuations, beginning at about $6^{n}$ p.m., is to be noted, which is somewhat similar to the one observed at the same weather station on July 15, 1970 (fig. 4 bis).

forma d'interazione con i disturbi microbarici. Ciò sta inoltre a provare che l'azione diretta del vento sulla diga è insufficiente ai fini della forma. zione di microsismi. 


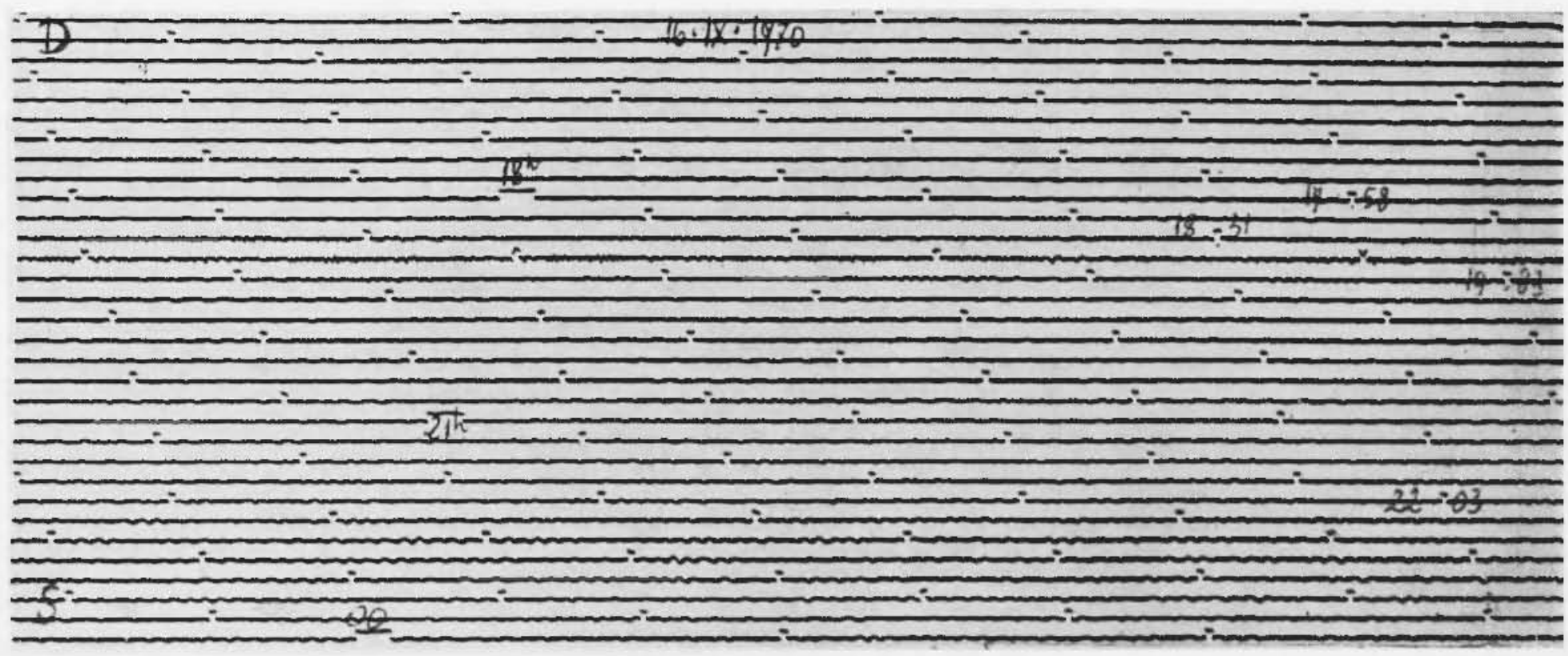

Fig. $9 a$.

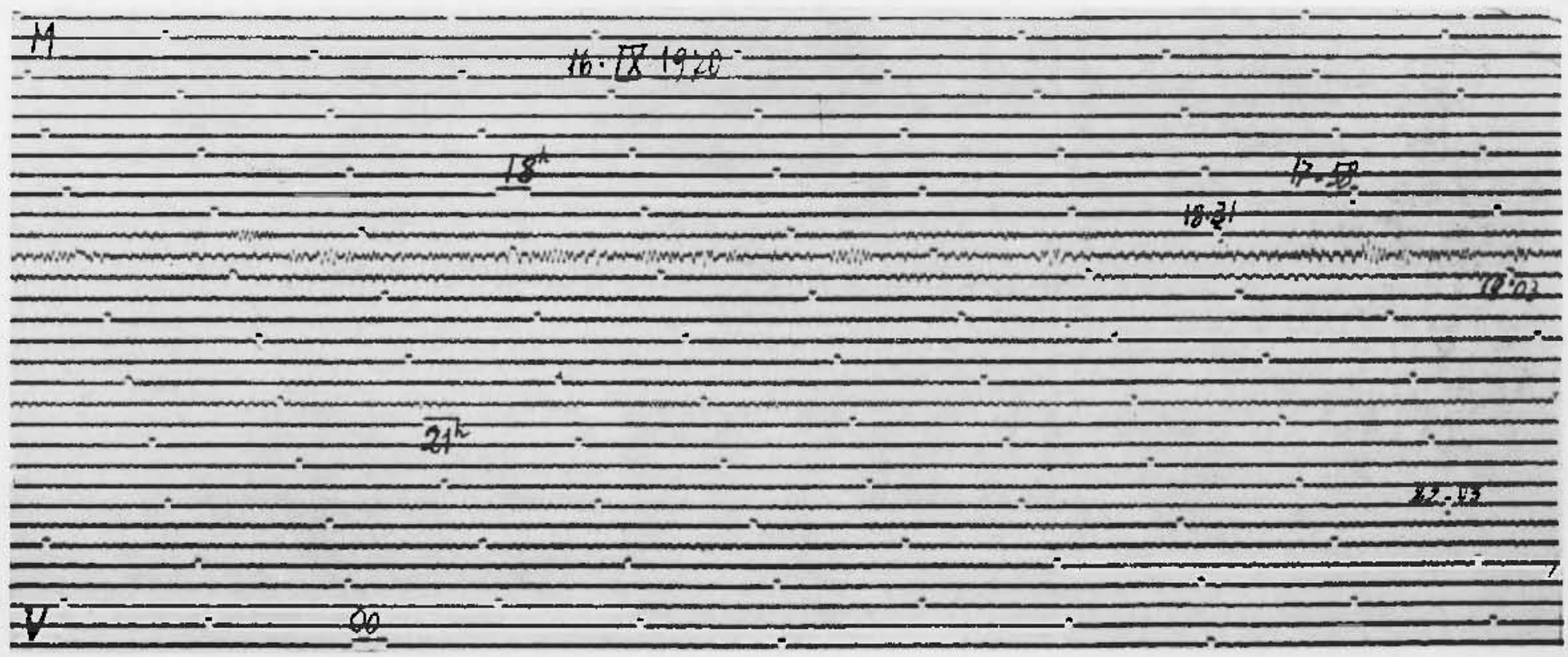

Fig. $9 b$.

Fig. 9 - Microsismi registrati a Pieve di Cadore in occasione del passaggio delia perturbazione del 16.IX.1970 (figg. 7-8). I microsismi provenienti dai lago appaiono nettamente meno sviluppati di quelli provocati dal cicione del 15 . VII. 1970. Anche i successivi microsismi provenienti dal golfo di Trieste in arrivo a Pieve dopo le $22^{\text {h }}$ (più cliaramente sulla comp. $D S$; la $V M$ aveva infatti perso di sensibilità nei suoi confronti) risultano molto meno sviluppatí degli analoghi provocati dal ciclone del 15.VII.1970. Del resto, anche il loro periodo -- dell'ordine di 2 sec - è leggermente inferiore a quello osservato il 15. VI1.1970. Ciò sta a provare che la tempesta microsismica, originata nel golfo di Trieste il 16.IX.1970, fu associata a sistemi di onde significative che non avevano ancora raggiunto il loro completo sviluppo.

Fig. 9 - Microseisms recorded in Pieve di Cadore on occasion of the passage of the perturbation of September 16, 1970 (figures 7,8). The microseisms coming from the lake appear distinctiy less developed than those brought about by the cyclone of July 15 , 1970 . Aiso the suecessive microseisms from the Gulf of Trieste, which reached Pieve after $10^{\mathrm{h}} \mathrm{p}$.m. (more discernable on the right-leit component, as the hill-valley component, as a matter of fact, had lost all sensitivity in its regari), resulted much less developed than the analogous ones caused by the cyclone of July 15, 1970. Jesides, also their period, in the order of 2 sec., is slightiy inferior to that observed on July 15, 1970. This is a proof that the microseismic sterm originating in the Gulf of Trieste on September 16 , 1970 was associated to significant waves systems which had not yet reached their apex. 


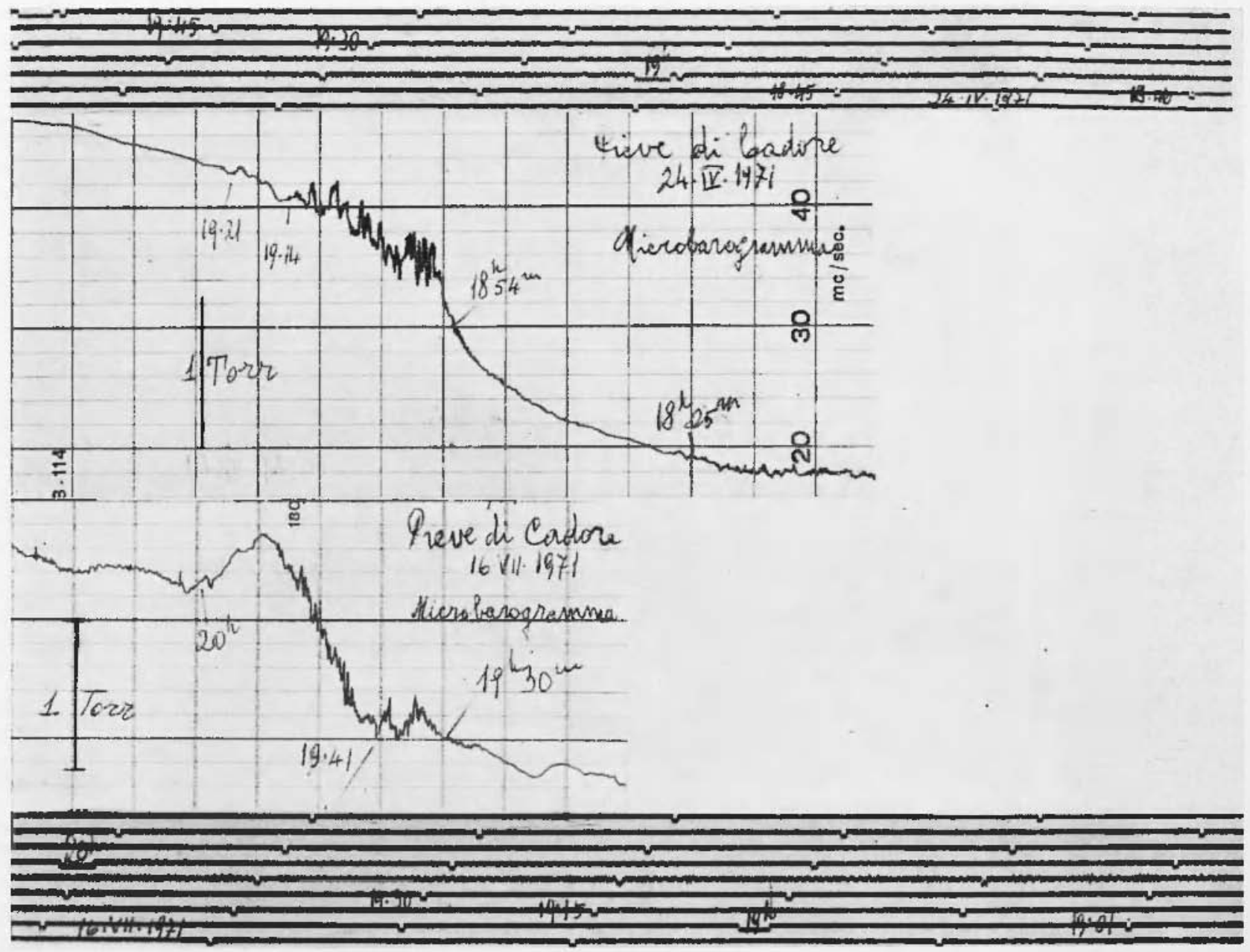

Fig. 10a - Lievi microsismi sulla comp. Valle-Honte $(\nabla M)$, in corrispondenza del passaggio di gruppi di rapile perturbazioni microbariche, sul lago di Pieve di Cadore, talle $19^{1 \mathrm{l}}$ ca alle $19^{\mathrm{b}} 20^{\mathrm{m}} \mathrm{ca}$.

Fig. $10 b-$ Microsismi dalle $19^{\mathrm{h}} 37^{\mathrm{m}}$ ca alle $20^{\mathrm{h}} 15^{\mathrm{m}}$ ca, associati al passaggio sul lago di lievi rapidi, disturbi microbarici, su pressione atmosferica in rapida ascesa. I microsismi iniziano sempre qualche minuto dopo

linizio dei disturbi microbarici o terminano aleuni minuti dopo il passaggrio del nueleo perturbante.

Fig. 10a - Light mieroseisms on the hill-valley component, corresponding to the passage of groups of rapid microbaric perturbations on the Lake of Pieve di Cadore, from about $7^{\text {h }}$ p.m. to about $8^{\text {1n }} \mathrm{p} . \mathrm{m}$.

Fig. $10 \mathrm{~b}$ - Microseisms from about $7^{\mathrm{h}} 37^{\mathrm{m}}$ p.m. to about $8^{\mathrm{h}} 15^{\mathrm{m}}$ p.m. associated to the passage of light, rapid microbaric perturbations over the lake. Hicroseisms always set in a fow minutes after the beginning of the microbaric perturbations and end a few minutes after the passage of the perturbing nuclens. 

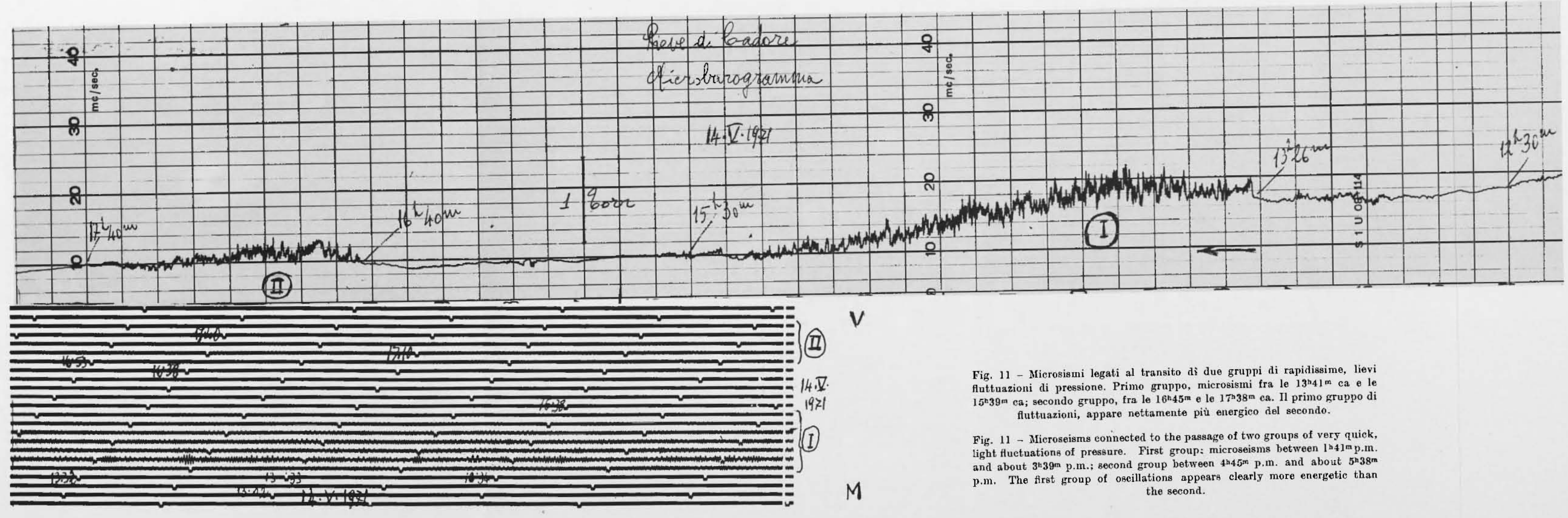

(1)

14.7

197

Fig. 11 - Microsismi legati al transito dì due gruppi di rapidissime, lievi Fig. 11 -

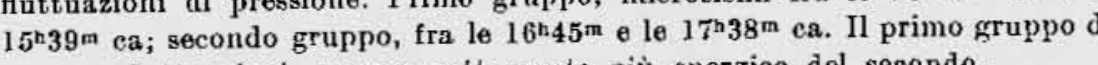
fluttuazioni, appare nettamente più energico del secon

Fig. 11 - Microseisms connected to the passage of two groups of very quick, light fluctuations of pressure. First group: microseisms between $\mathrm{l}^{\mathrm{m}} \mathrm{l} \mathrm{l}^{\mathrm{m}} \mathrm{p} . \mathrm{m}$. and about $3^{\text {nt3 } 39 \mathrm{~m}}$ p.m.; second group between
p.m. The first group of oseillations appears clearly more energetic than the second. 


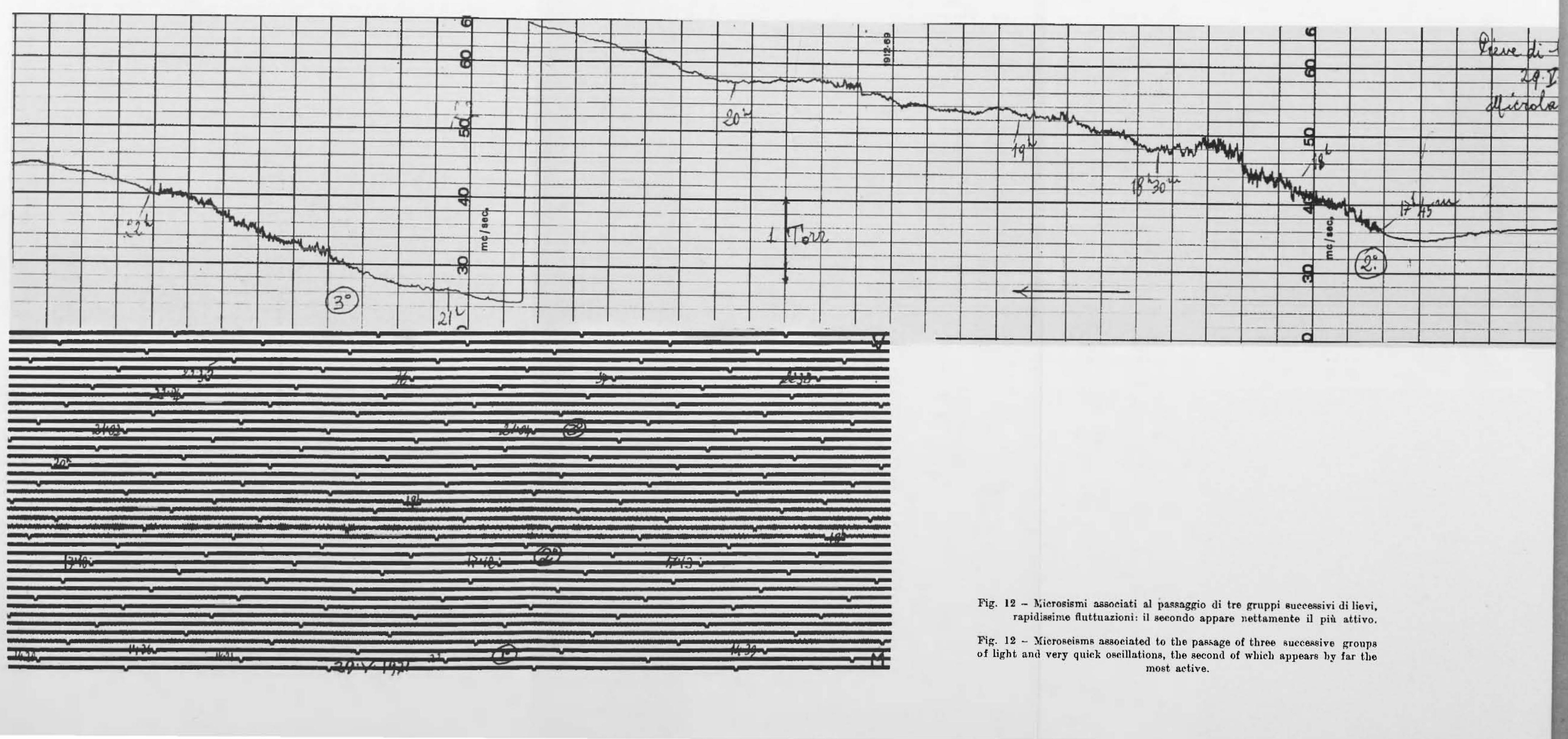




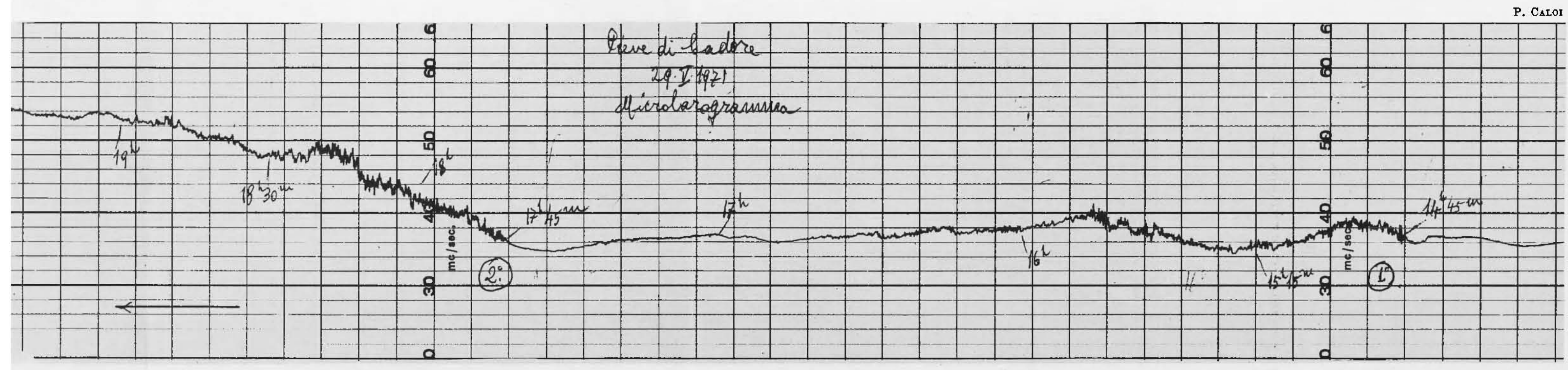

Fig. 12 - Nicrosismi associati al passaggio di tre gruppi successivi di lievi, rapidissime fluttuazioni: il secondo appare nettamente il più attivo. Fig. 12 - Microseisms associated to the passage of three successive groupe most active. 


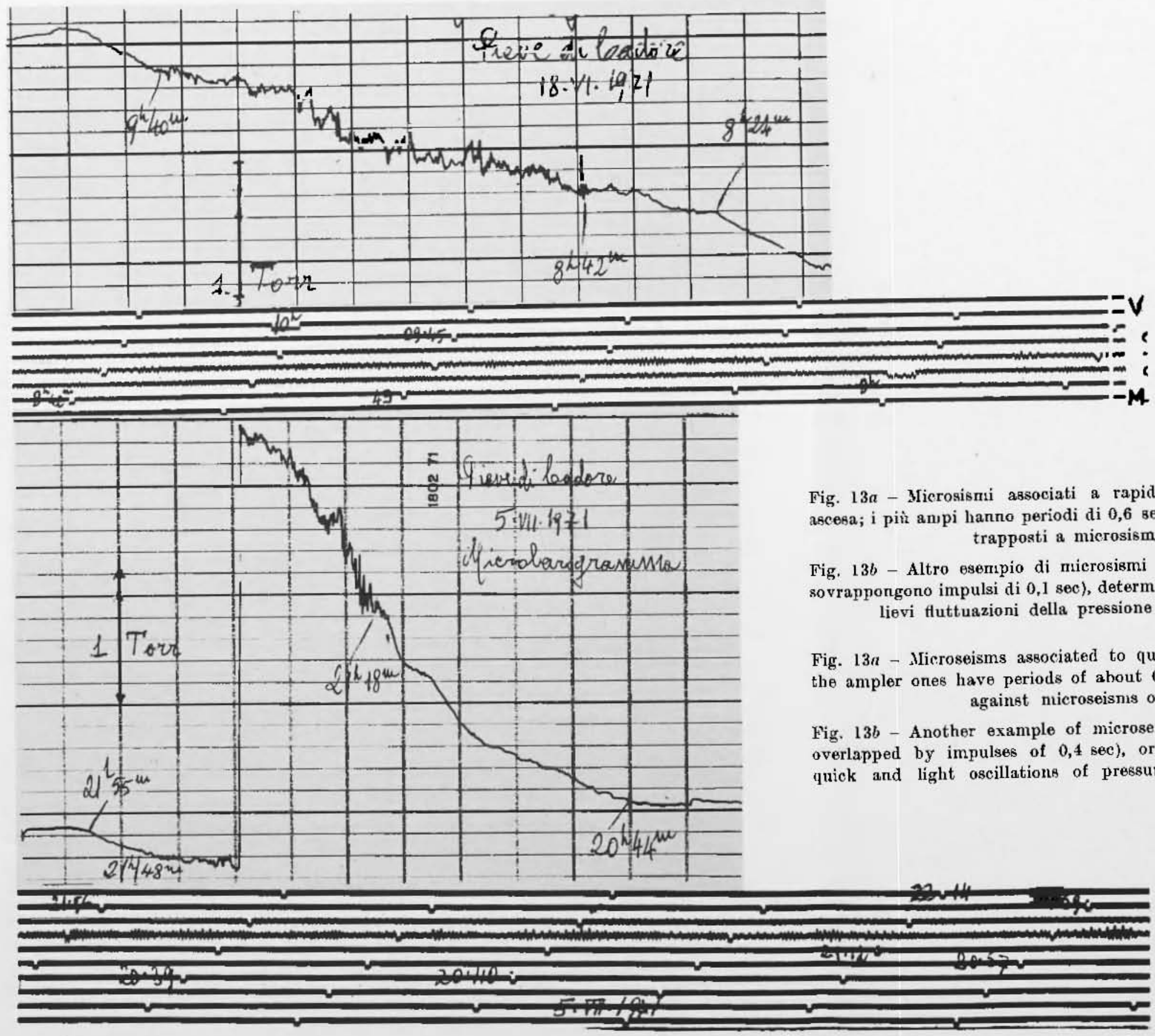




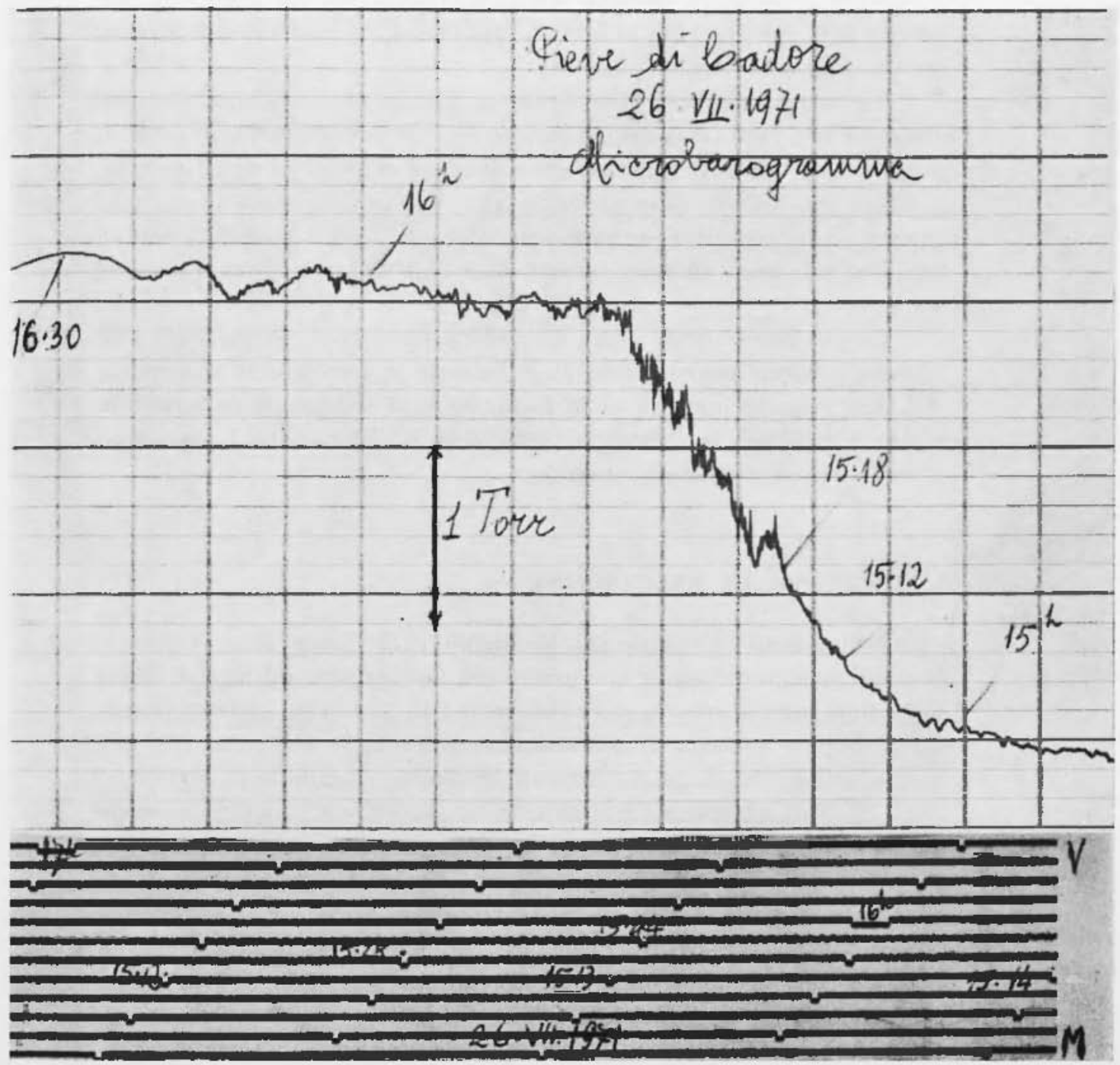

Fig. 14 - Il transito sul lago di rapidissime, tenui fluttuazioni microbariche, su pressione in brusca ascesa, provoca la registrazione di lievi microsismi (con inizio verso lo $15^{\mathrm{h}} 2 \mathrm{l} \mathrm{m}$ sul ritmo di $4 \mathrm{sec}$ ca, cui si sovrappongono impulsi di 0.4 sec).

Fig. 14 - The passage of very quick and light microbaric oseillations over the lake, on a pressure of sudden rise, gives recordings of light microseisms starting at about $3^{\mathrm{h}} 2 \mathrm{I}^{\mathrm{m}}$ p.m. at intervals of about 4 seconds, which are overlapperl by impulses of $0-4$ sec. 
Saturalmente, si possono avere microsismi per la durata di parecchie ore, quante durano lo fluttmazioni della pressione atmosferica, rla eui traggono origine (fig. 18).

Ia ancora messo in forte evidenza il fatto che le tempeste microsismiche dal lago iniziano generalmente con periodi dell'or(ine di 3 , 1 o s sec, ai quali si sovrappongono quelli di alcumi decimi di secondo. Localmente, quindi, possomo origiuare - come si è visto per i mari interni e gli Oceani $\left({ }^{4}\right)$ - microsismi dei più diversi periodi, associati alla varietà degli impulsi agenti nelle fluttuazioni flella pressione atmosferica.

Infine, va osservato che i nicrosismi tal lago di Pieve tli Cadore levono ritenersi provocati da onde forzate: le piccolissime rlimensioni del lago impediscono infatti la formazione di sistemi di onde significative e rendono ben lieve la probabiliti di formazione delle condizioni per ma risonanza cinetica.

\section{HICROSISMI DA MARI INTWRNI.}

Ritorniamo al ciclone del 15 Luglio 1970. Dopo aver provocato la registrazione di vistosi microsismi nel suo transito sul lago di Pieve di Cadore, proseguendo la sua corsa verso $\mathrm{SF}$, giunto sul golfo di Trieste dava origine a cospicui microsismi, caratteristici del golfo (periodo merlio dell'orliue di 25,7 ), secondo modalità esaminate in precerlenza $\left({ }^{3}, 4\right)$. Tali microsismi, animati da un'energia nettamente superiore a quella associata ai microsismi del lago "Caclore n, si propagano a distanze dell'ordise di alcune centinaia di chilometri. La loro propagazione uon avviene però in modo uniforme; essa appare fortemente condizionata dalla natura geologica regli strati esterni clella crosta. I microsismi vengono infatti fortemente esaltati dalla Val Padana (v. registrazioni di Padova, Bologna e Pavia - figg. 21, 22, 23 dove funzionano strumenti, con piccolissimo ingrandimento), presentano normale attenuazione nel settore $\mathrm{NW}-\mathrm{NE}$, llove ginngono, sia pure con ampiezza molto rirlotta fino a Vienna (figg. 23, 24), e trovano uno sbarramento pressochó invalicabile in corrisponcienza degli Appennini.

Ia perturbazione ciclonica del 16 Settembre 1970 fu cii intensità molto minore. I microsismi da essa provocati sia a Pieve di Cadore sia nel golfo di Trieste risultarono pertanto di minore ampiezza (figg. 9, 26, 27). Anche il raggio della zona interessata dai microsismi 


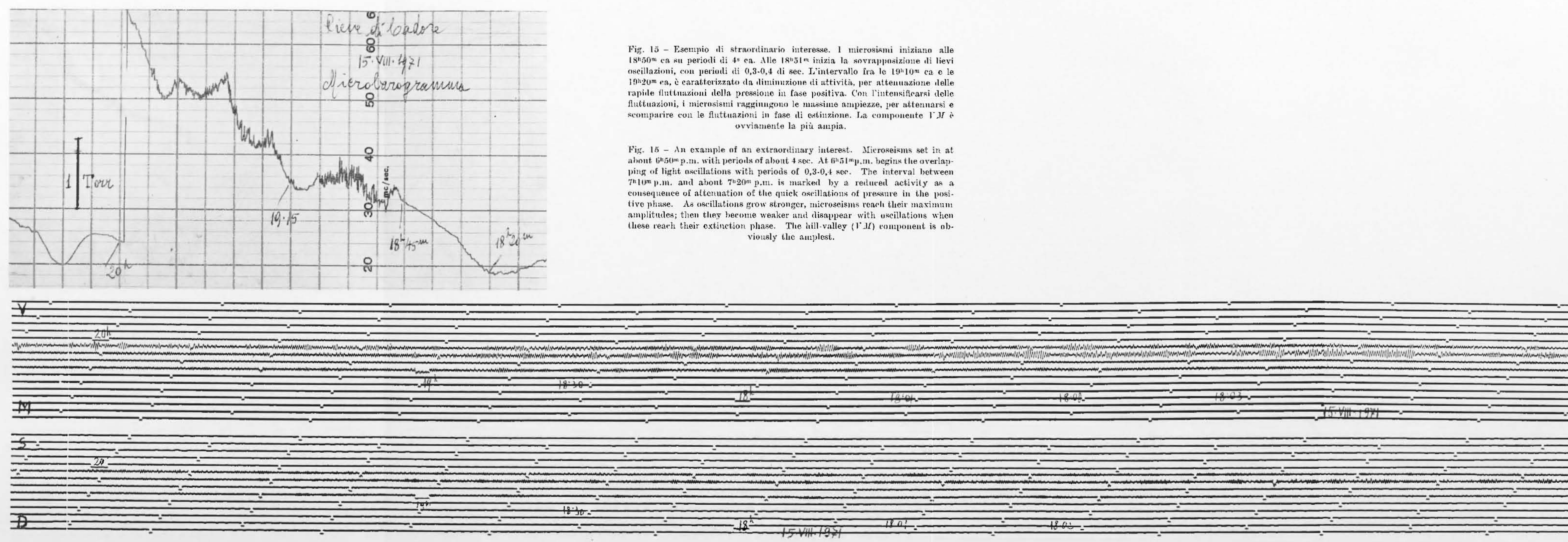




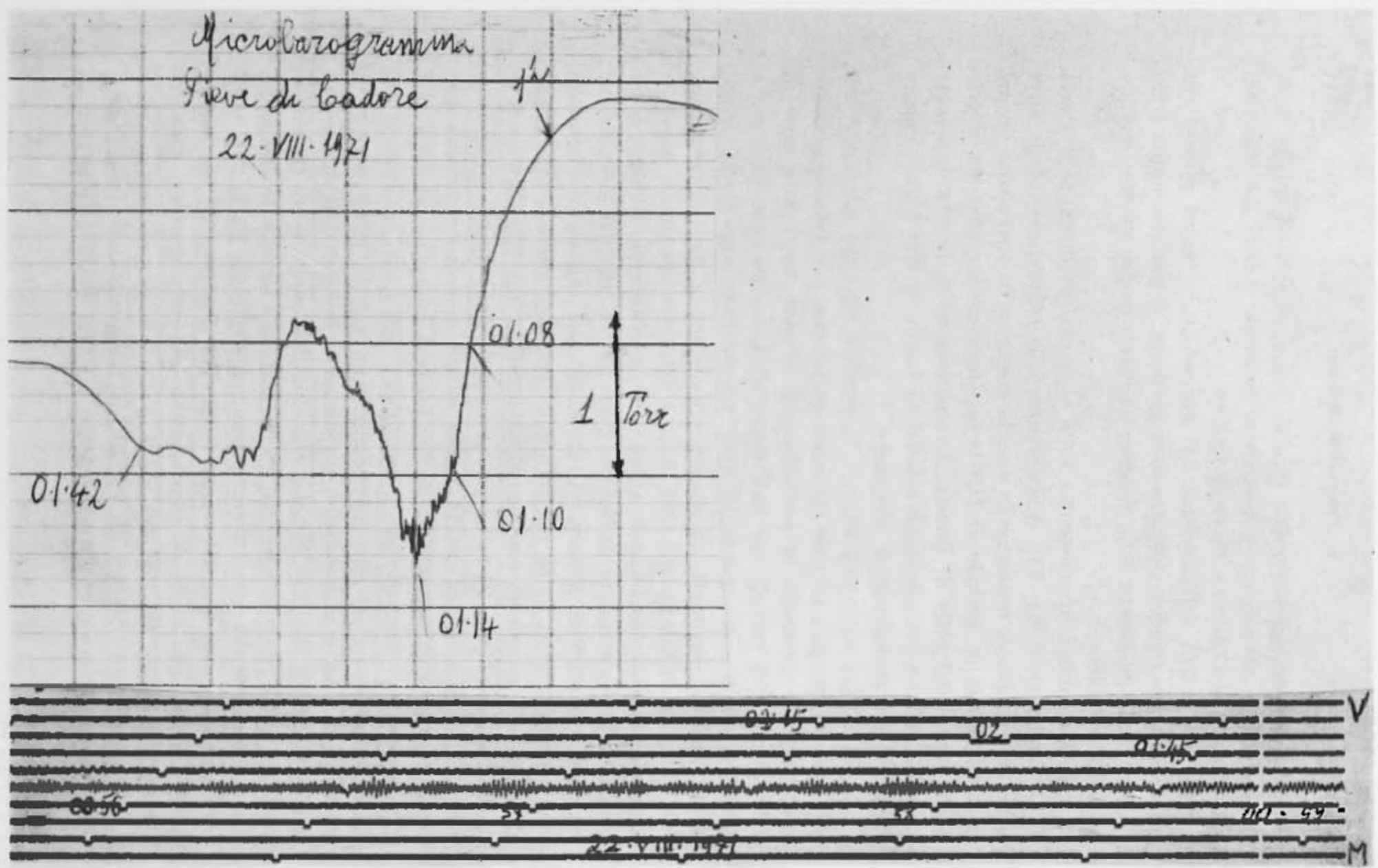

Fig. 16 - Altro notevole esempio di mierosisni, associati a rapidissime fluttuazioni della pressione, in tran*ito sul lago. Fig. 16 - Another noteworthy example of microseisms, associated with very quick pressure oscillations passing over the lake. 
adriatici appare quindi molto ridotto, nei confronti di quello della perturbazione precedente: i mierosismi risultano infatti già attenuati a Somplago, Ia Maina e Pieve di Cadore.

Dal rapporto clell'ampiezza dei microsismi, ottenuti presso una stessa stazione sismica nelle clue clate preclette, si puó avere una chiara idea della differenza delle energie, animanti le due diverse manifestazioni eicloniche.

Soflermiamoci un momento sulle registrazioni ottenute a Pavia il 15 Luglio 1970 (fig. 23). A differenza delle registrazioni delle altre stazioni esaminate, noteremo in esse la presenza di mierosismi fin dal mattino, e con un periodo di $3 \mathrm{~s}, 3-4^{\mathrm{s}} \mathrm{ca}$, superiore a quello dei microsismi propri del golfo di Trieste. La spiegazione di questa ajparente anomalia si ha osservando che, gì dal 14 Tuglio, il Mar Ligure appare investito da perturbazioni cicloniche.

Come è già stato osservato (3. $\left.{ }^{1}\right)$, stazioni sismiche sistemate sulle coste, ai margini di zone di mare comunque perturbato, possono registrare, in occasione di perturbazioni intense, tutta una serie di mierosismi, con periodi che dall'ordine di 1 sec possono arrivare a valori di quelli delle coperiodali onde significative: sono questi ultimi i microsismi persistenti, di massima ampiezza e che si propagano alle maggiori distanze. Gli altri, hanno carattere sporalico, associati a sistemi di onde temporanee, animati da poca energia. Ebbene, come risulta dalle registrazioni di Genova (figg. 28-30), in quel giorno e nel successivo la stazione sismica ivi funzionante ha segnalato microsismi dall'aspetto caotico, come risultato della contemporanea registrazione di tutta una gamma di oscillazioni, associate al mare agitato da perturbazioni cicloniche. Di tutta questa congerie di oscillazioni, griungono a Pavia, dalle 9 in poi del 15 Luglio ed ivi vengono registrate con carattere di regolaritì, solo quelle con periodo dell'ordine di $3,5-4$ sec: tali oscillazioni costituiscono i microsismi persistenti, originanti nel Har Ligure, associati alle coperiodali oncle marine significative. Sismografi di pari caratteristiche, registrano a Bologna tali microsismi molto attenuati (fig. 22), benché essa non clisti dalle coste tirreniche molto di più di quanto non disti Pavia. Ciò sta a significare che, mentre i microsismi del Mar Ligure trovano una finestra fra le Api e gli Appennini, per propagarsi verso l'alta Val Padana, gli Appennini costituiscono una sorta di sbarramento alla propagazione verso la restante valle del Po (fig. 31).

Intorno alle $19^{\mathrm{h}}$ del 15̃.VII.70, ai microsismi che provengono dal golfo di Genova, si sovrappongono a Pavia quelli di provenienza 


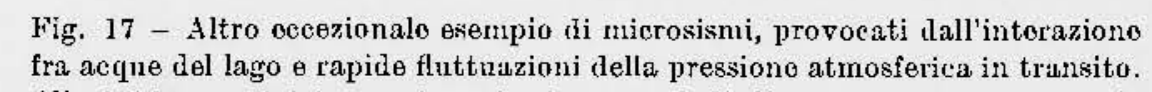

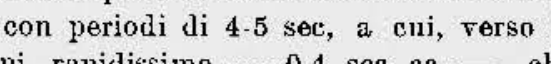

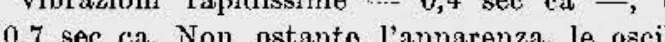

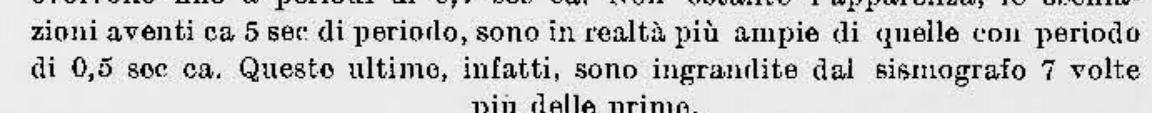

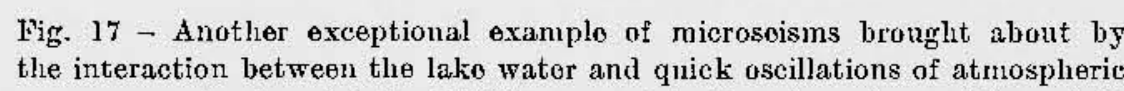

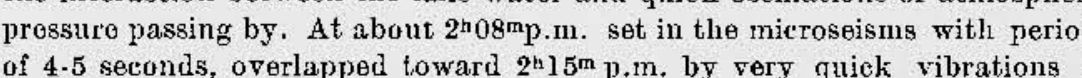

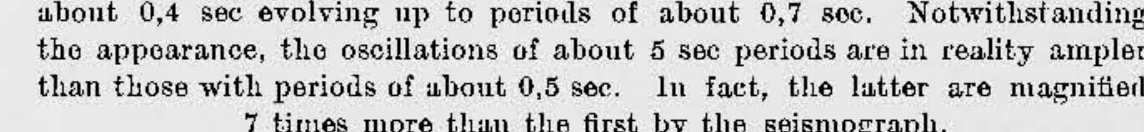

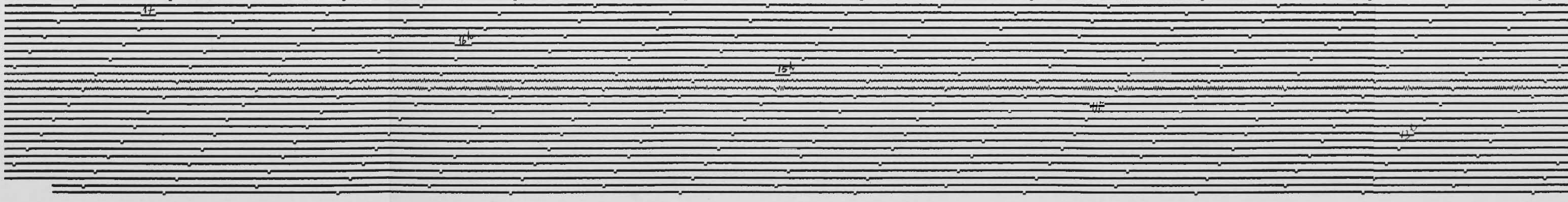




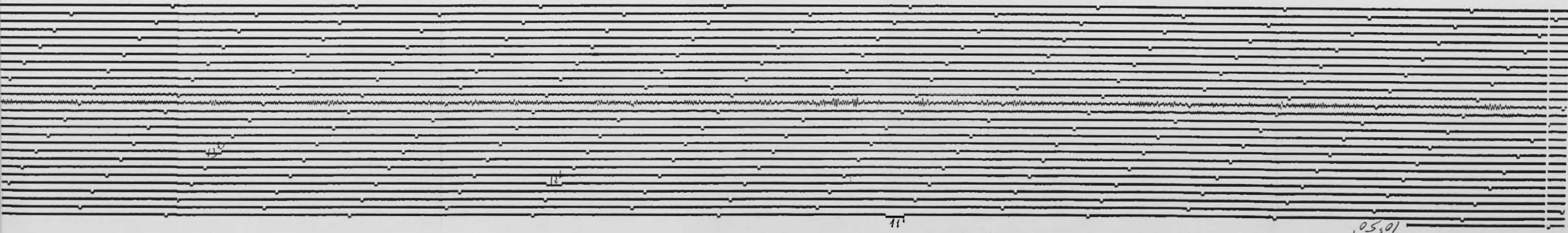



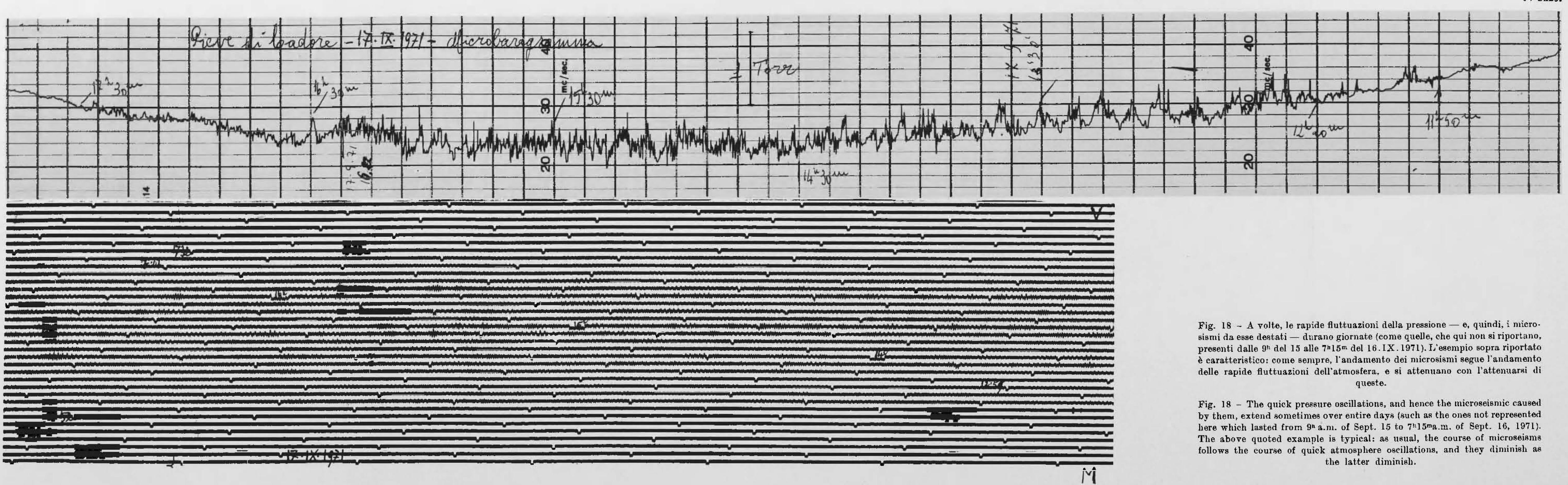

Fig. 18 - A volte, le rapide fluttuazioni della pressione $-e$, quindi, i nicro.

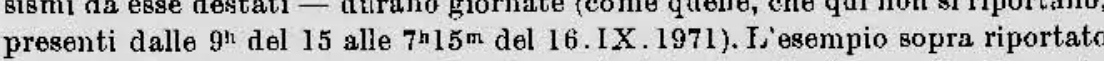

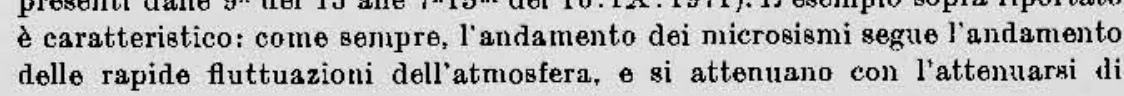

Fig. 18 - The quick presesure oscillations, and hence the microseismic caused

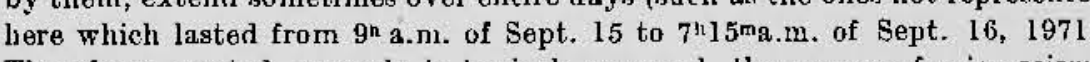

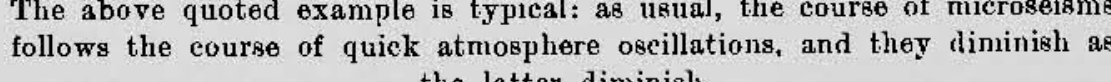


adriatica: essi risultano di periodo dell'ordine di $2^{s}, 7$ e sono di piccola ampiezza. Se ne conclude che Pavia è particolarmente sensibile ai microsismi, provocati da perturbazioni del Mar Ligure.

2.1 - Queste distinzioni fra microsismi persistenti, provenienti da diversi centri-origine, è resa possibile dal fatto che, nella loro propagazione, i microsismi non subiscono dispersione $\left({ }^{3 .}{ }^{4}\right)$.

Ia perturbazione che ha determinato la tempesta microsismica nel Mar Ligure, proseguendo la sua corsa lungo il Tirreno, provoca la registrazione di microsismi, sporadici e persistenti, anche nella stazione sismica di Roma Città Universitaria (fig. 31).

$\mathrm{E}$ interessante osservare la diflerenza delle registrazioni microsismiche ottenutc contemporaneamente a Genova e a Roma, durante il transito di questa tempesta. A Genova l'agitazione microsismica raggiunge la massima intensità fra le $13^{\text {h }}$ ca e le $22^{\text {h }}$ ca (tempo di Gr) ed ha carattere esclusivamente locale: l'aspetto caotico (figg. 28$30)$, sta a significare la sovrapposizione di oscillazioni per sistemi d'onde sporadici, temporanei: gli pseudo-periodi infatti non superano i $3 \mathbf{s}$.

A Roma microsismi apprezzabili iniziano verso le $15^{\text {th }}$ del 15 .VII ed aumentano via via di ampiezza, per raggiungere i massimi verso le $6^{\text {h }}$ del 16.VII ed oltre. Sembra quindi esservi uno sfasamento con Genova; ma solo apparente. Si noti che a Roma i microsismi presentano periodi dell'ordine di 4-5 sec, contro i 2 s in media di Genova. La spiegazione è di carattere strumentale. Gli apparechi a breve periodo di Genova presentano ingrandimenti dinamici che variano, in media, da 8.000 a 3.500 per periodi da $1^{*}$ a 3 , mentre l'ingrandimento dinamico di movimenti con periodi da $4^{\mathrm{s}}$ a $5^{\mathrm{s}}$ decresce da 600 a $300{ }^{(1)}$.

Pertanto, l'eventuale presenza di microsismi con periodi superiori a 4 sec, a Genova viene generalmente mascherata da perturbazioni a più breve periodo, di carattere locale, amplificati una diecina di volte e più. A Roma (Città Universitaria) i sismografi GalitzinWilip presentano caratteristiche opposte: piccolo ingrandimento per brevi periodi (400-600 per periodi da 1 a 2 sec) e ingrandimento dell'ordine di 1500 per periodi da 4 a 5 sec.

E nostro parere che microsismi con periodi dell'ordine di 4-5 sec originano in corrispondenza della vasta zona di mare fra la Capraia, l'isola d'Elba e Livorno. Tale zona, caratterizzata dá piccole profondità del mare e delimitata in modo da formare come un ampio 


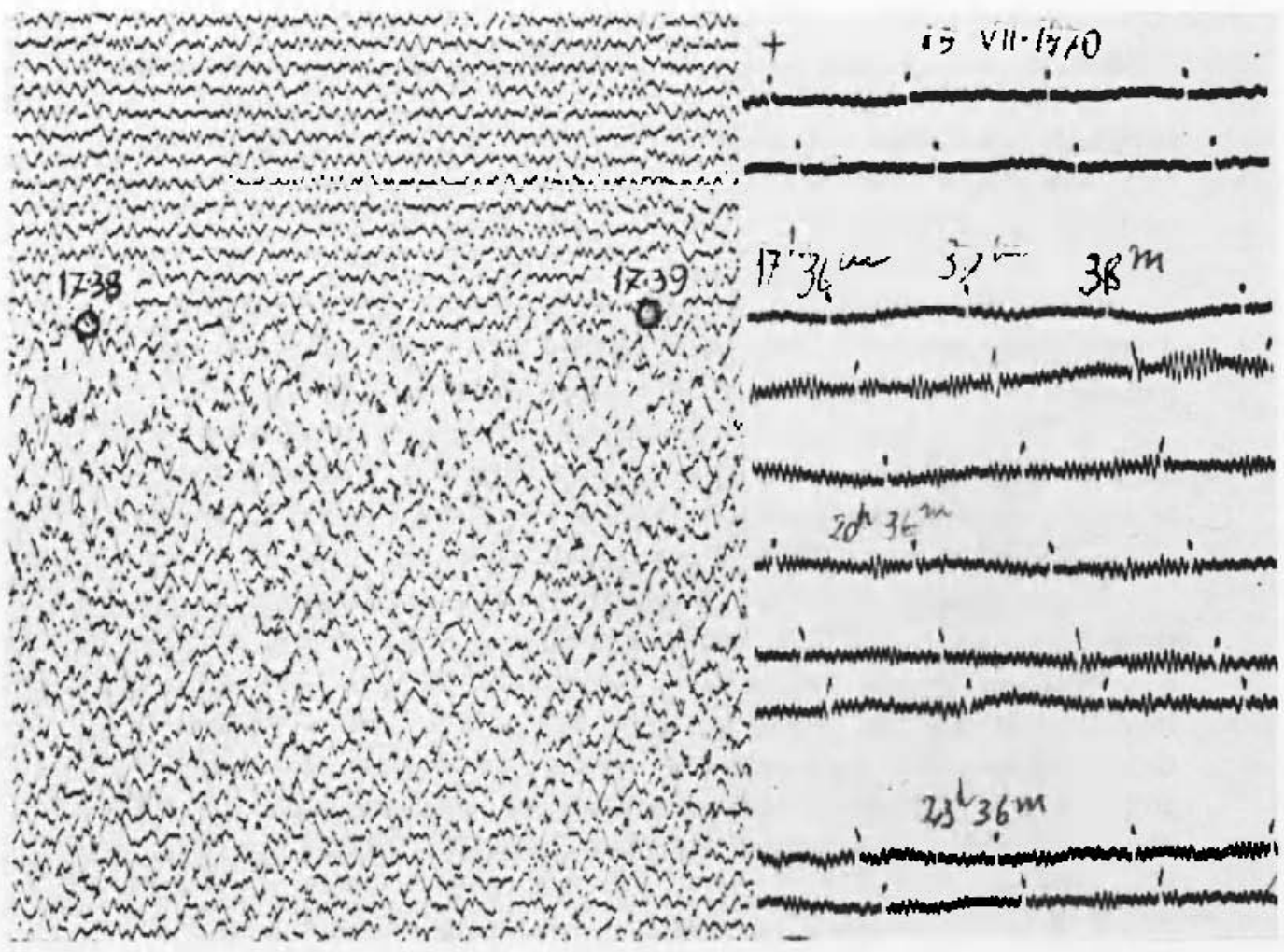

Fig. 19 - Rogristrazione dei microsismi, associati al transito del ciclone del 15.V11.1970 sul golfo ali Vonezia, of tenuta a Trieste. A sinistra figura la rogistruzione formita clalla comp. $E W$ a brove periodo $\left\langle T_{0}=1 s, 0 ; T_{q}-05,75\right)$; essa appare (aotica, con prevalenza di impulsi sli periorli varianti di 1 a 2 sec ea. Questi non sono $i$ microsismi fondamentali, aventi un periodo sli $2 \mathrm{~s}, \mathbf{b} \mathrm{ea}$, bensi perturbazioni a carattere socomlario, sporaklico, lovate a sistenti onlosi transitori. Essi mascherano i microsismi fondamentail, legati a sistemi onlosi permanenti, "significativi ", quaii sono appunto guelli registrati slai sismografi a lungo periodo (a dest ra della figura: comp. $Z$, con poriodi strumentali $T o=15^{\mathrm{s}}, 1, T g=100$ sec); sul ritmo di 2s,6 ca. Questa disparita li reristrazione ò di carattere strumentalo: i sismografi a breve periodo ingrandiscono iufatti le perturbazioni con periodi dell'ordine di 1 sec, migliaia rli volte di più di yuelie con periodi intorno ai 3 soc, mentro i sismografi a lungo periorlo hanno un ingrandimento dinamico pressoché costante per periorli da 1 a 3 sec. Pertanto, puelli con periodo clell'ordine di $2^{3}, 7$ sono i microsismi fondamentali, eccitati ral cicione iu transito sul golfo li Trieste: solo essi, infatti, vengono suecessivamente regristrati - con tale poriodo - a Somplago, Ta Maina, Paslova, Vajont, Pieve di Cadore, Bologna, Zagabria, fino alle massime slistanze (Vienua).

Firg. 19 . Recording of microseisms associaterl to tho pansage of the cyclone of . Iuly 15,1970 over the Gulf of Venice, takon in Trieste. On the left hand the recording obtainerl by the EWW component of a short poriod $\left(T_{0} \ldots[5,0 ; T g=0,7 \overline{5})\right.$ is shown; it appears chaotic, and impulses with periods varying from 1 to about 2 sec are prevailing it. These are not the funlanental mitroseisms with periods of about $2 \mathrm{~s}, 6$, but seconslary, sporadic perturbations due to transient wave systems. They camouflage the fundamental microseikms boumd to permanent, "significant " wave systems such as the ones recorded by the long period seismogruphs (on the right hand of the firure: $Z$ component with instrumental periods $\left(T o=15^{\mathrm{s}}, 1 ; T g=100 \mathrm{sec}\right)$ with rates of about $2 \mathrm{~s}, 6$. This recorkling diserepancy is due to the iustrument: the short period soismographs enlarge the perturbations of alout 1 sec periorls thousanls of times more than those liuving periorls of about 3 sec, while the long periou seismographs have a virtually constant slynamic magnifieation for periorls from 1 to 3 sec. The nicroseisms with periorls in the orkler of $2^{8}, 7$ are, therefore, the fundamental ones exciterl by the transient cyclone, over the Guif of Trieste. They alone are successively recorrlenl with the sail period in Somplago, La Maina, Parlova, Vajont, Pieve di Cadore, Bologna, Zagabria up to the maximum distances (Wien). 


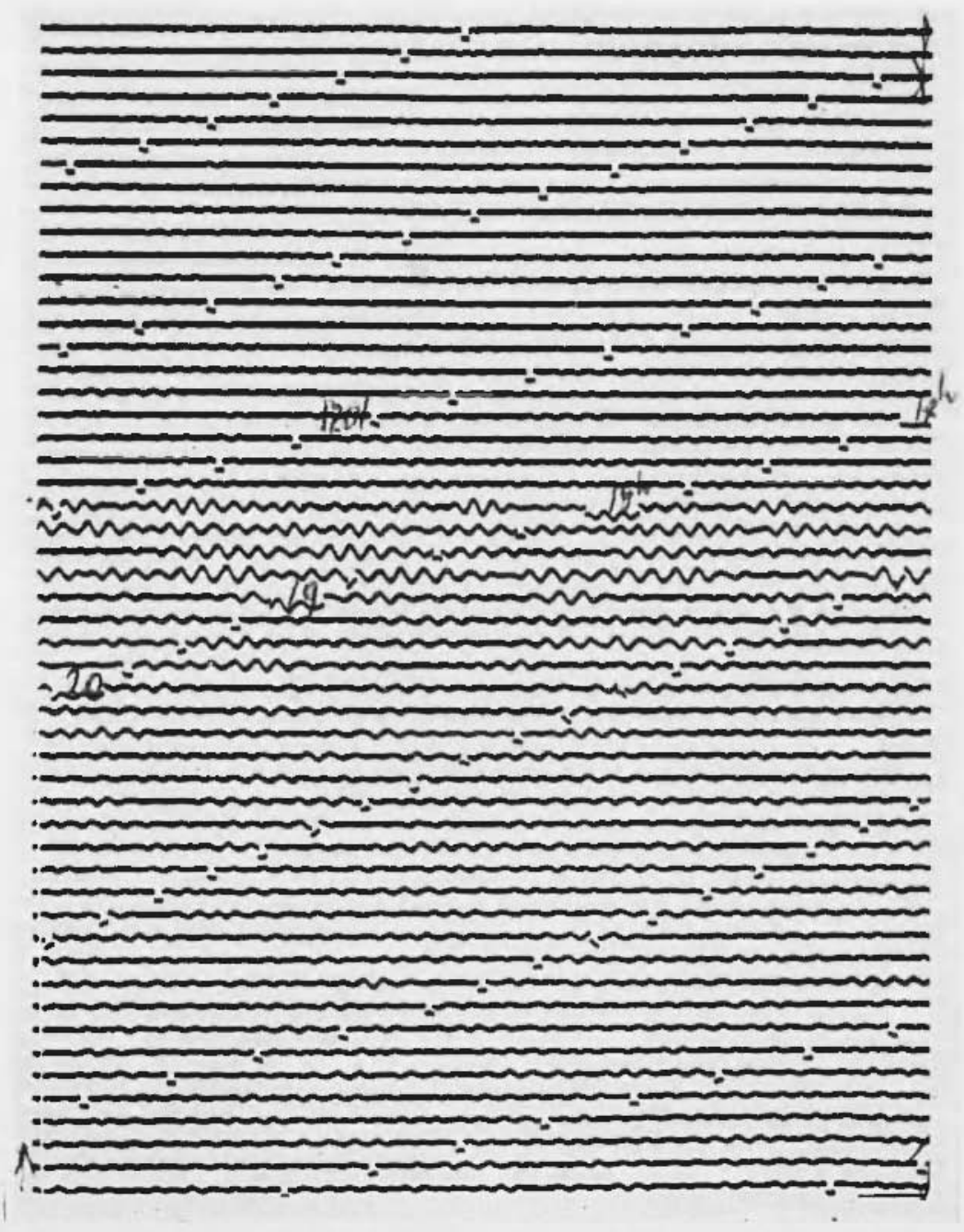

Fig. 20 - Registrazione dei microsismi provenienti dal golfo di Trieste, ottenuta sulla comp. NW.SW della staziono sismica di Somplago (presso il lugo di Cavazzo), a cirea $8 \tilde{\mathrm{km}}$ di distanza dalla zona-origine. I microsismi conservano il periorlo medio di 2 s, 7 , osservato a Trieste (ciclone del 15 . VII. 1970).

Fig. 20 - Recording of microseisms from the Gulf of Trieste, taken on the NE-SW component of the Somplago Seismic Station (near the Lake of (avazzo), at about $85 \mathrm{kms}$ listance from the origin area. Microseisms maintain the moan period of $2^{s}, 7$ observerl in Trieste (cyclone of July 15,1970 ). 


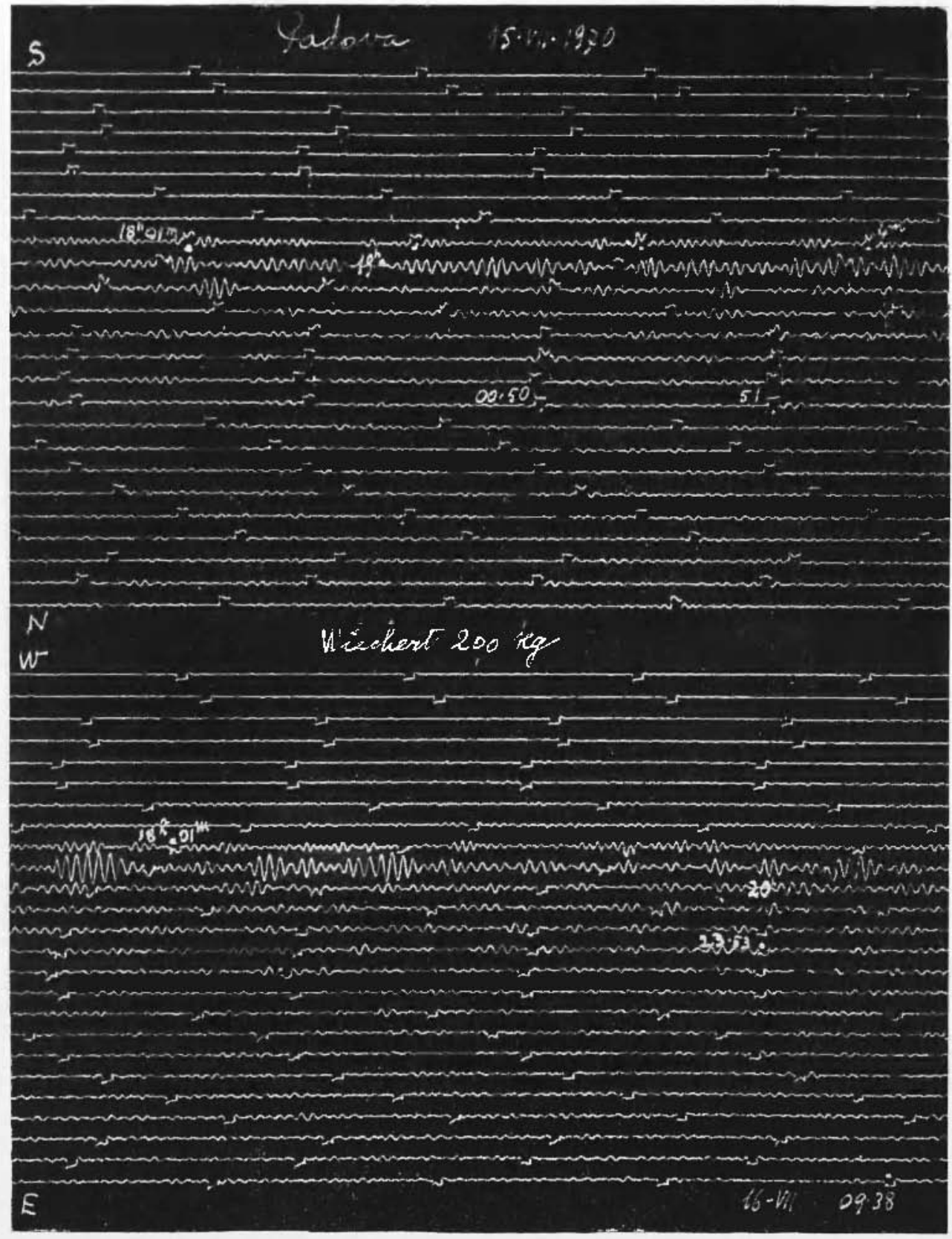

Fig. 21 - Registrazione di microsismi, associati al transito del ciclone del 15.VII.1970 sull'alto Adriatico, ottenuta a Padova, ad oltre $130 \mathrm{~km}$ dalla zona-origine (golfo di Trieste). La Val Padana esalta questo tipo di oscillazioni. I microsismi registrati a Padova risultano infatti nettamente più ampli di quelli contemporaneamente ottenuti a Trieste, ai margini della zona-origine. Va precisato cle il "Wiechert " di Padova ha un ingrandimento pari a meno di $1 / 5$ di quello del sismografo a lungo periodo funzionante a Trieste (fig. 19), per periodi doll'ordine di 3 sec.

Fig. 21 - Recording of microseisms associated to the passage of the cyclone of July 15, 1970 over the High Adriatic Sea, taken in Padova at over $130 \mathrm{kms}$ distance from the origin area (Gulf of Trieste). The Po Valley enhances this kind of oscillations. The microseisms measured in Padova, in fact, are distinctly ampler than those mea. sured at the same time in Trieste at the margin of the origin area. It must be added that the "Wiechert" of Padova has a magnification of less than $1 / 5$ of the magnification of the long period seismograph, that is for periods in the order of 3 sec, installed in Trieste (Fig. 19). 
golfo (fig. 32) nei confronti di perturbazioni provenienti da NW, consente l'apprestamento delle condizioni dinamiche, atte ad esaltare la formazione di microsismi ("). Riteniamo ehe, in tale zona, le onde signifieative possano raggiungere, nel loro pieno sviluppo, i 4-6 sec e provocare sul fondo l'insorgere di microsismi coperiodali, quali appunto vengono registrati a Roma (C.U.).

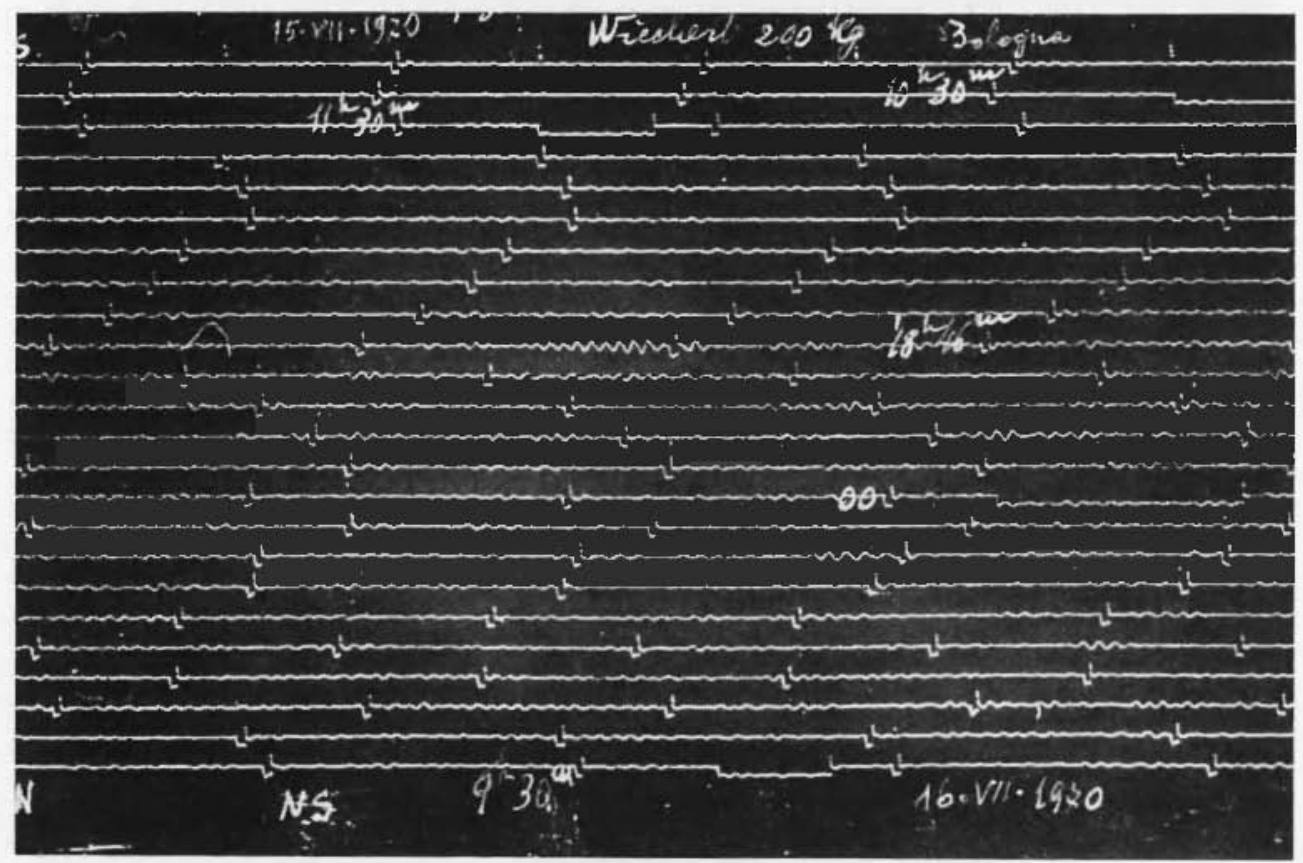

Fig. 22 - I microsismi provocati nel golfo di Trieste dal ciclone del 15 . VII. 1970 vengono chiaramente registrati anche a Bologna, dal piccolo "Wiechert" locale, ad oltre $200 \mathrm{~km}$ di distanza dalla zona-origine.

Fig. 22 - The microseisms caused in the Gulf of Trieste by the cyclone of July 15, 1970 are clearly recorded also in Bologna by the small local " Wiechert", at over $200 \mathrm{kms}$ distance from the origin area.

Per le ragioni dette, tali microsismi passano generalmente inosservati a Genova, salvo nei casi in eui presentino ampiezza cospicua e l'agitazione locale sia di piccola intensità (fig. 30, parte superiore); non appena però l'agitazione locale riprende a svilupparsi, scompare a Genova (fig. 30) ogni traccia di microsismi di $5^{\text {s }}$ ca, contemporaneamente registrati a Roma (C.U.). 


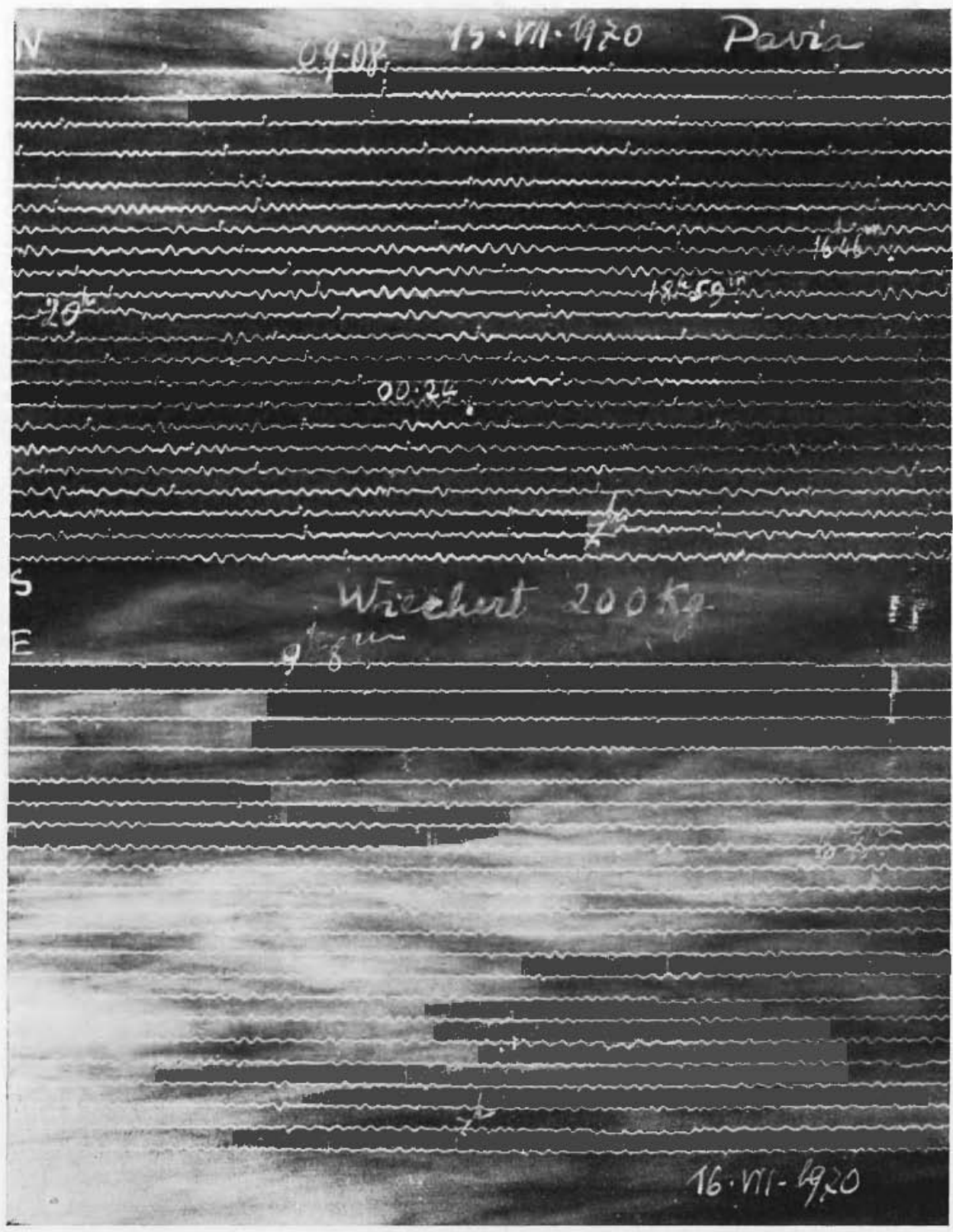

Fig. 23 - Dopo le $19^{\text {h }}$ (ora solare) del 15. VII.1970, anche sul "Wieclert " $200 \mathrm{~kg}$ di Pavia (a ca. $340 \mathrm{~km}$ di distanza) si ha registrazione di microsisini provenienti dal golfo di Trieste. Essi però sono proceduti (e, in gran parte, sopraftatti) da microsismi (con periodi dell'ordine di $3^{\mathrm{s}}, 5-3^{\mathrm{s}}, 7$ ) provenienti dal Mar Jigure, ed ivi provocati dalla stosa perturbazione, in transito parecchio ore prima (fig. 2).

Fig. 23 - After 7" p.m. (Solar Time) of July 15, 1970, the $200 \mathrm{~kg}$ "Wiechert" of l'avia, too, at about $340 \mathrm{kms}$ distance, gave a recording of mieroseisins from the Gulf of Triesto, preceded, however, and covered to a large extent by microseisms with periods in the order of $3^{\mathrm{a}}, 5-3^{\mathrm{a}}, 7$ from the Ligurian Sea where they had been caused when the same perturbation passed there a few hours earlier. 
L'aumento del periodo da 4 a o sec ca non è dovuto a fenomeno di dispersione (che, come sappiamo, è pressoché nullo nei microsismi) ma all'aumento del periodo dei sistemi di onde marine significative, da cui i microsismi prendono origine. Questo, quando la tempesta. microsismica supera certi limiti di durata $\left({ }^{\circ}\right)$. ì un modo diverso per esprimere il concetto, piì volte accennato: il periodo tei microsismi, ciò, aumenta tino a raggiungere il massimo valore in corrispondenza del completo sviluppo del sistema di onde significative.

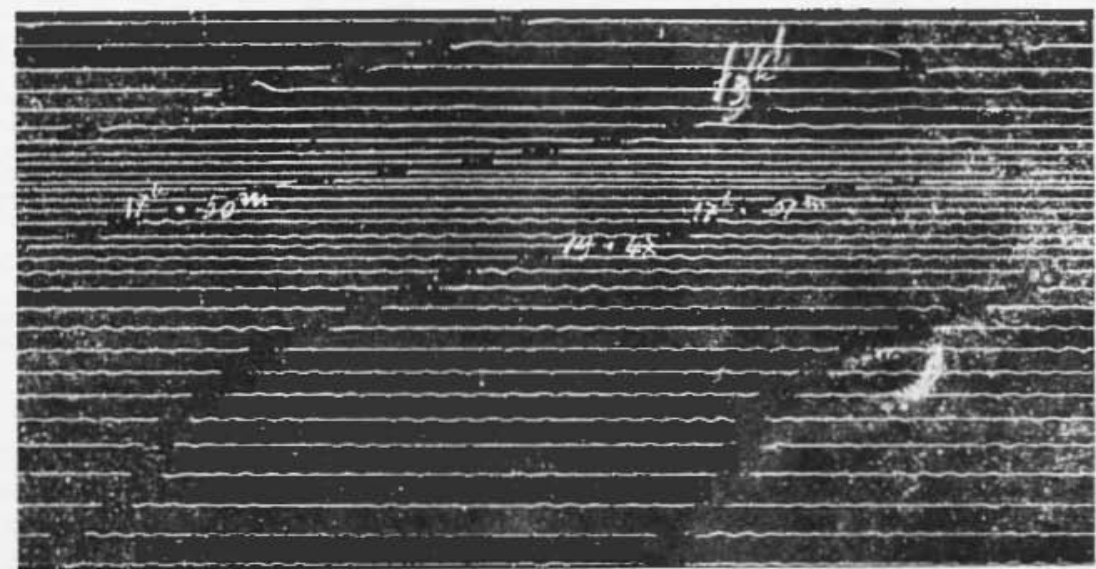

Fig. 24 - I microsismi provenienti dal golfo di Trieste (15.VII. 1970) ven. gone registrati a Zagabria, a ca $200 \mathrm{~km}$ di distanza dalla zona-origine. Si noti che la loro ampiezza è nettamente inferiore a quella registrata a Bologna (fig. 22), alla stessa distanza, malgrado il "Wiechert" $1300 \mathrm{~kg}$ di Zagabria amplifichi tali movimenti circa il doppio del piccolo "Wiechert " di Bologna. Nuova testiunonianza sull'effetto amplificante della Val Padana su movimenti del genere $\left(T=2^{\mathrm{s}}, 7 \mathrm{ca}\right)$.

Fig. 24 - The microseisms from the Gulf of Trieste (July 15, 1970) are recorded also a Zagabria, at some $200 \mathrm{kms}$ distance from the origin-area. It is to be noted that their amplitude is clearly smaller than that recorded at Bologna (fig. 22), at the sarne distance, although the $1300 \mathrm{~kg}$ "Wiechert" of 7agabria gives about twice the amplification of movements in comparison to the small "Wiechert" of Bologna. This is another evidence of the amplifying effect of the $P_{0}$ valley on the movements of this kind $(T=26,7$ approximately).

Per quanto afferisce alla relazione fra periodo delle onde marine e periodo dei microsismi, possiamo osservare che, per onde che "sentono il fonclo n, i periorli dell'onda liquida e di quella solida coincirlono, 
mentre per acque profonle l'elietto sul fondo porta al dimezzamento del periodo dell'onda di mare agente, in conformità alla teoria di Junguet-Higsins.

\section{Microsishi bajlóogano atuantioo.}

Faremo pocle aggiunte a quanto è già stato seritto cla uno di noi ${ }^{\left({ }^{4}\right)}$. I microsismi di provenienza atlantica traggono la loro origine dal transito, da ovest ad est, di vaste aree depressionarie, che, in prossimità delle coste europee, interagiscono con le acque del sottostante ocemo. I periodi elevati $(7-9$ sec $)$, si spiegano con la formazione di

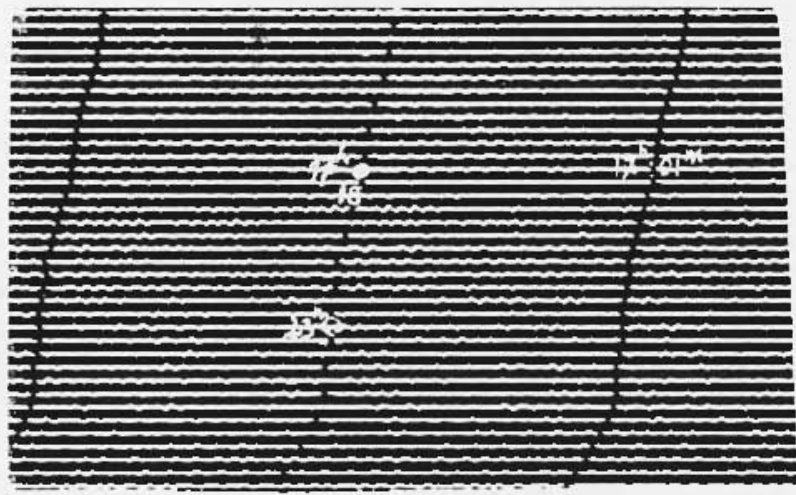

Fig. 25 - Anche a Vienna -- a ea $355 \mathrm{~km}$ di distanza - gimgono i micro. sismi, askociati al transito del ciclone del 15.VII.1970 sul golfo di Trioste. Come a Trieste, e nelle altre stazioni d'osservazione, l'agitazione presenta dei massint verso le $18^{\mathrm{h}} 30^{\mathrm{m}}$ (ora di Greenwich), conserva il medio periodo della zona-origrine $(2,7 \mathrm{ca})$, ma $\dot{e}$ fortemente ridotta in ampiezza.

Figr. 25 - Evel Wien, at about $355 \mathrm{kms}$ distance, was reached by the microseisms associater to the passage of the cyclone of July 15, 1970 over the rinlf of Trieste. As was the case in Trieste and the olher observation stations, the agitation showed peaks toward $6^{\mathrm{n}} 30^{\mathrm{m}} \mathrm{p} . \mathrm{m}$. (Greenwich Time), maintains the mean period of the origin area $\left(2^{5}, 7\right.$ approx. $)$, but its amplitude is strongly reducod.

"fetch" estesi, nei quali le onde significative possono ragginngere il loro completo sviluppo: $\dot{e}$ in corrispondenza di esse che nascono sul fonrlo microsismi di pari periorlo. I'esaltazione di tali microsismi è ra attribuire a fenomeni di risonanza cinetica, fra le onde significative 
c coperiodali fluttuazioni barograficlie, presenti nella pressione atmosferica, in fase positiva ( $\left.{ }^{4}\right)$.

Naturalmente, anche nelle zone-origine atlantiche, il complesso movimento ondoso locale dà la stura presso le coste ad un'estesa gamma di perturbazioni microsismicle, capaci di mascherare anche i microsismi persistenti, di maggior periorlo (fig. 36). Peró, a qualche distanza dalle zone d'insorgenza, solo questi ultimi continuano la propagazione, che può superare alcune migliaia di $\mathrm{km}$ di tragitto:

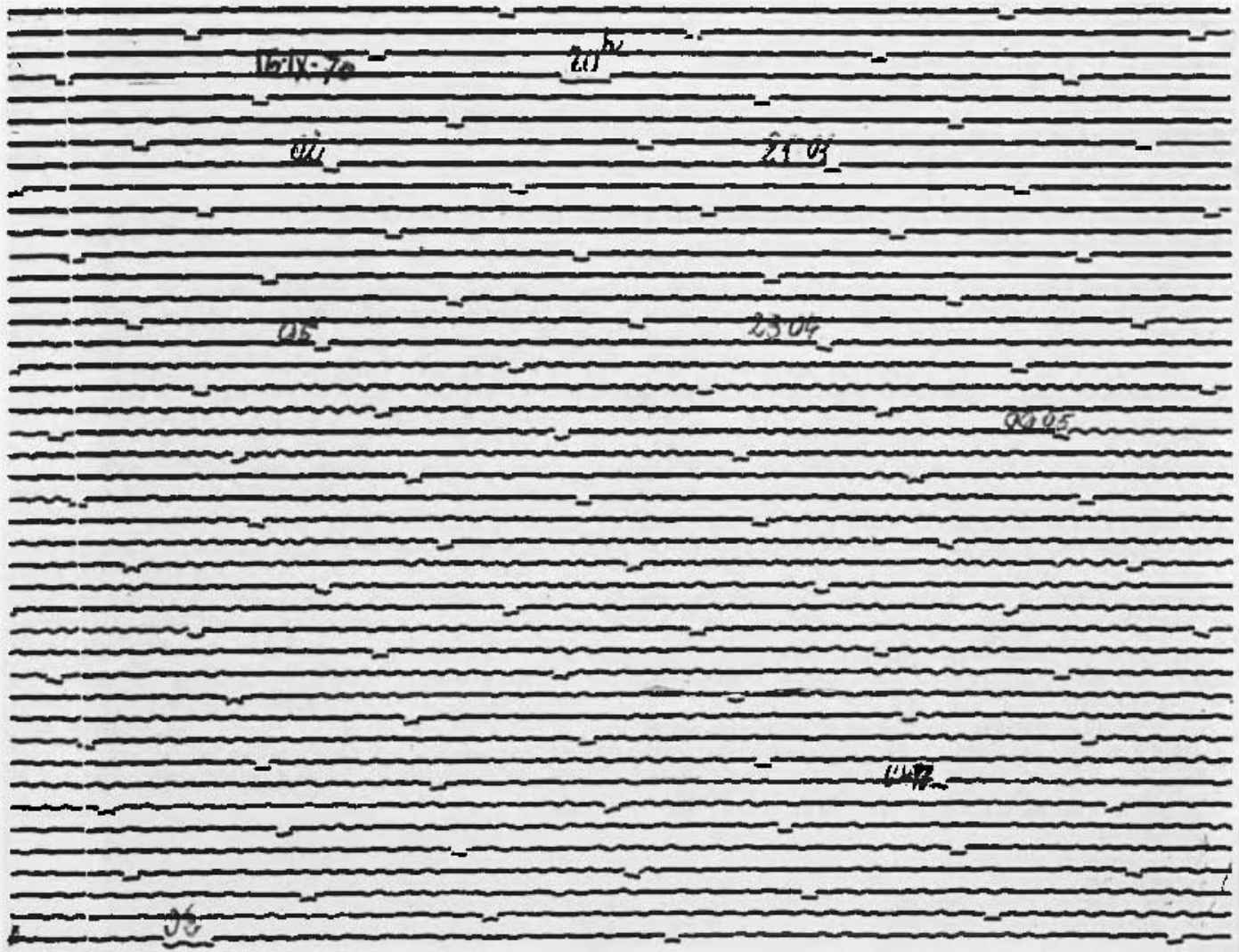

Fig. 26 - Microsismi provenienti dal golfo di Trieste, associati alla perturbazione del 16. [X. 1970 (figg. 7, 8), registrati a La Maina (Sauris), a partire dalle 23' ca (ora solare), alla distanza di $110 \mathrm{~km}$ ca dalla zona-origine (v. fig. 9).

Fig. 26 - Microseisms from the Gulf of Trieste, associated to the perturbation of Sept. 16, 1970 (figures 7 and 8), recorded at La Maina (Sauris) beginning at about $11^{\mathrm{h}}$ p.m. (Solar Time) at a distance of about $110 \mathrm{kms}$ from the origin-area (see fig. 9). 


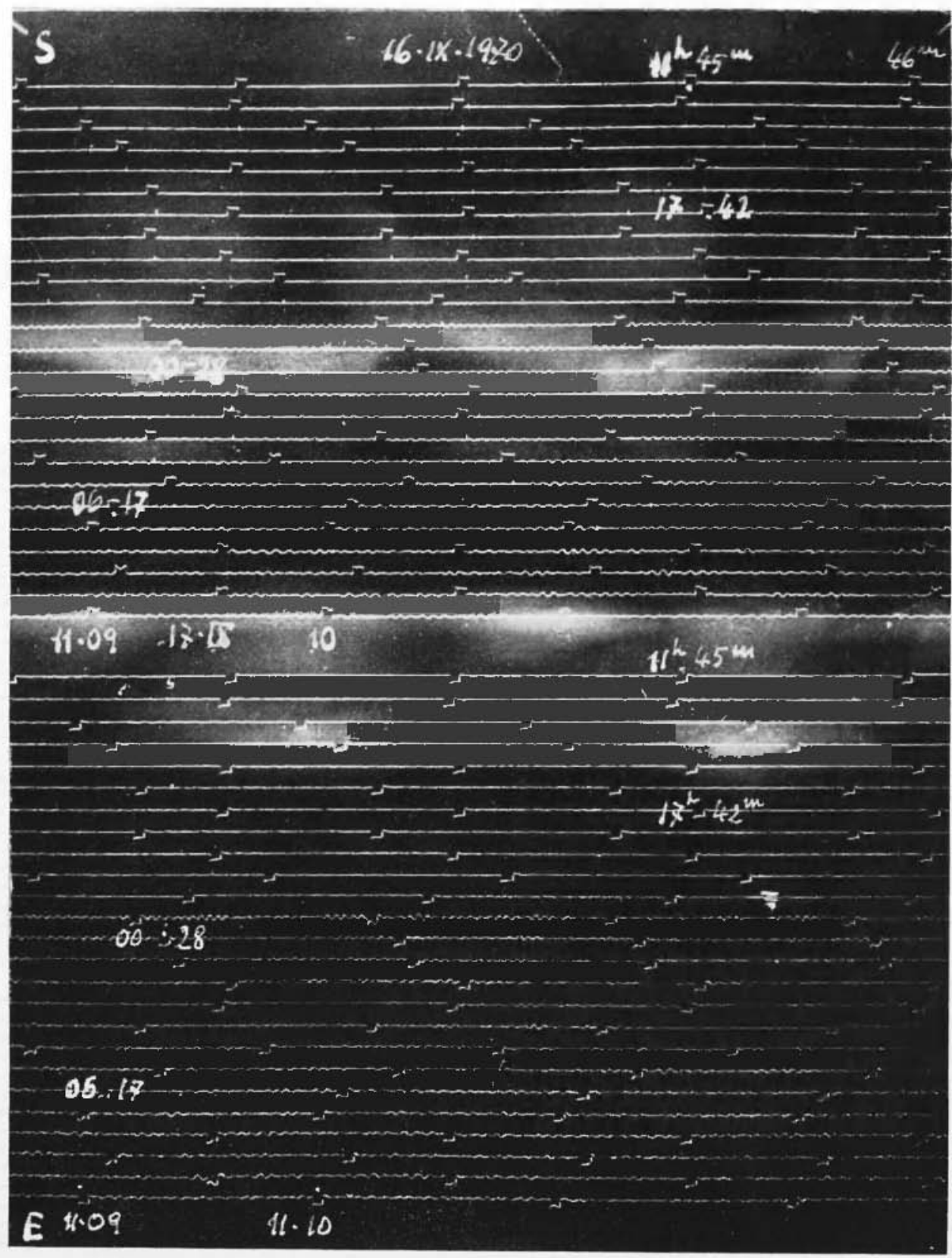

Fig. 27 - Perturbazione del 16.IX.1970, di cui alle figg. 7 o 8. Microsismi registrati a Palova, ad oltre $130 \mathrm{~km}$ di distanza dlalla zona-origine. Come di consueto, per efietto della coltre della Val Padana, particolamente sensibile a questi novinenti, a Padova -. malgrado il piccolo ingranclimento del sismosgrufo locale - I $\mathrm{am}$ piezze appaiono più accentuate che a la Maina, dove funziona un sismografo ad amplificazione nettamente maggiore.

Fig. 27 - Perturbation of Sept. 16, 1970, as per figures 7 and 8 . Microseisms recorded at Padova at over $130 \mathrm{kms}$ of distance from the origin-area. As usually, under the effect of the cover of the Po Valley, which is particularly sensitive to these movements, and in spite of the linited magnification of the local seismograph, the amplitules recorted in Purlova are more accentuated than those of La Maina where a seismograph of a sizeably larger anplification is installed. 


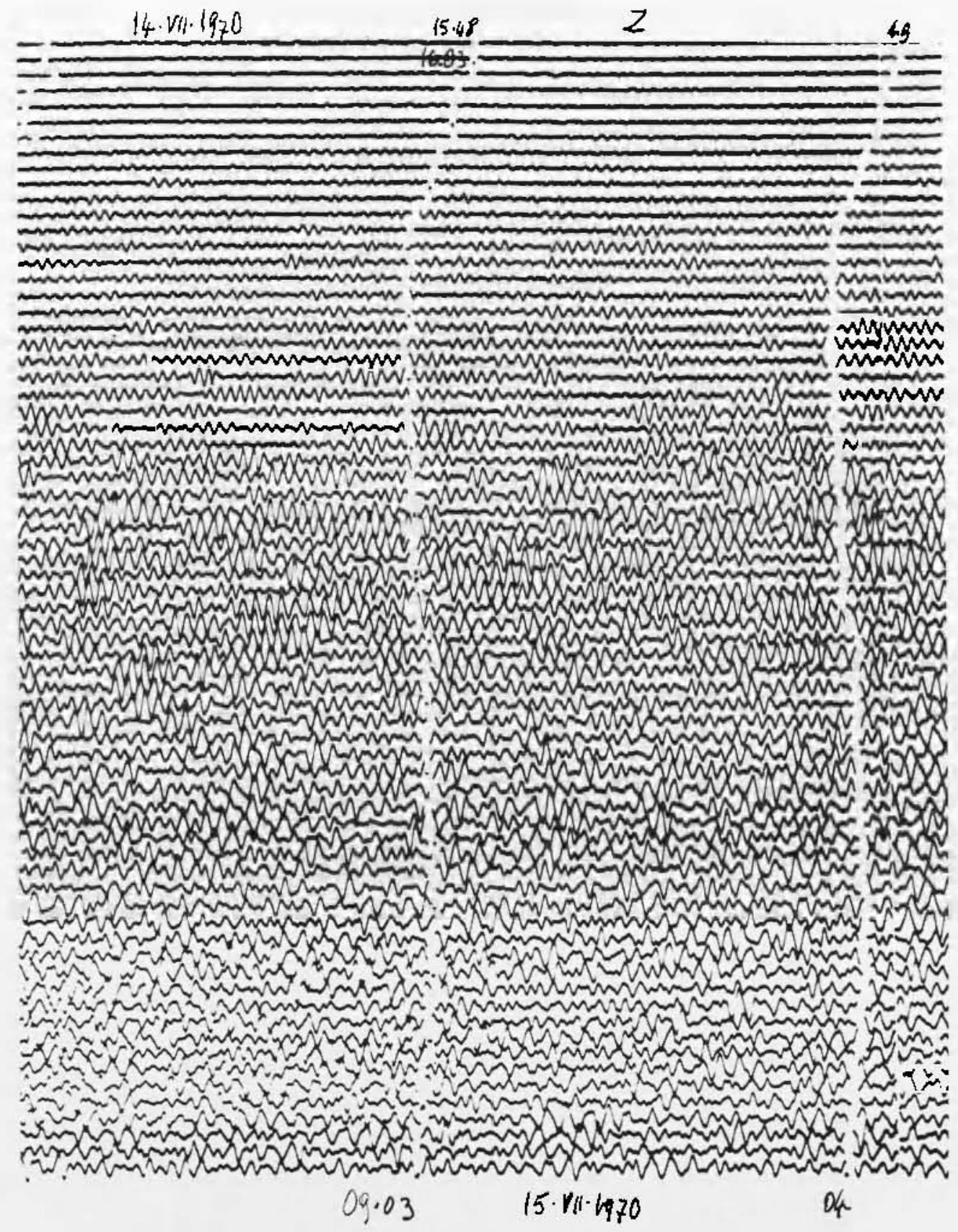

Fig. 28 - La perturbazione del 15. VII .1970) (fig. 2) ha cominciato ad interessare il Mar Ligure dalla notte precedente. I lievi microsismi regolari con perioslo da 1.5 a 2 sec, sono sla attribuire all'agitazione nella zona di mare prossima alla costa. Il carattere cantico che la registrazione assume a partire slalle prime ore tlel 15 , è da attribuire alla sovrapposizione sli microsismi, generati da sistemi onlosi sporulivi, temporanei agenti nella zona sli La spezia. Il procedere della perturbazione nella vasta zona di mare poco profondo fra La Spezia e l"isola d'Elba da origine ai microsismi foudamentali kuoi propri, con periodo dellordine si $5 \pm 1$ sec, chiaramente regristrali a Roma (fig. 31), mentre a Genova uli strumenti a breve periodo continuano a registrare microsismi di minor lunglezza donda, lesgati a sporadici, temporanei moti slel mare (figg. 29-30).

Fig. 28 - The perturbation of July 15, 1970, has reached the Ligurian Sea in the preceding night (Fig. 2). The light regular microseisms of a period between 1,5 ans 2 see must be attributed to the anitation in the sea area near to the shore. The clavotic aspect of the reeorling since the first hours of July 15 is the result of overlappings of microseisms originating from sporadic wave systems acting temporurily in the area of La Spezia. As the perturbation proceeded in the vust shallow sea area between La Spezia und Elba Island, it gave rise to fundumental microseisms peculiar to it, with periods in the orler of $5 \pm 1$ sec, which were clearly recorlesl in Rome (fig. 31 ), while the short periosl instruments installes in Genou continued to rorord microseisms of shorter wave lengthss, connected to sjoradic ankl temporary 
15. Vif. 197009.09

NS

Q9.10

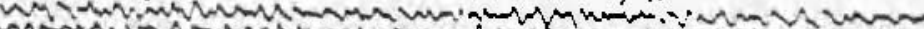

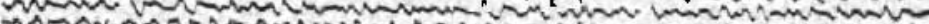

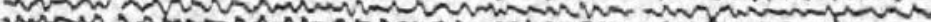
a

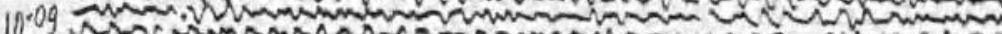

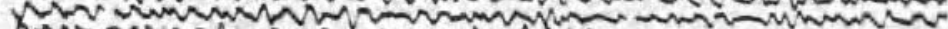

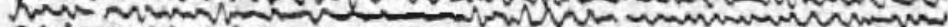

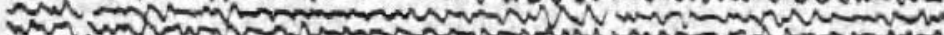

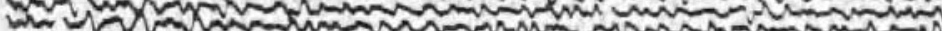

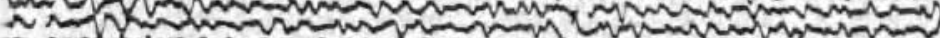

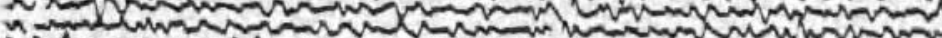

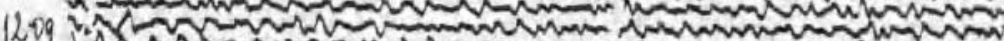
E) Co com mand 5 -

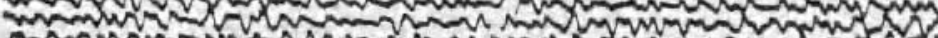

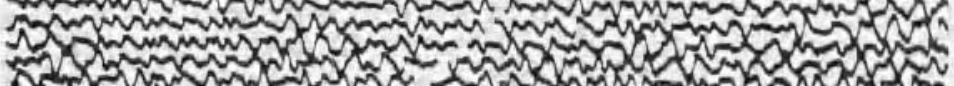

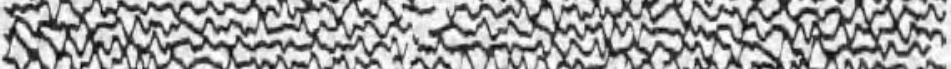

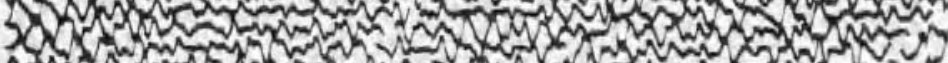

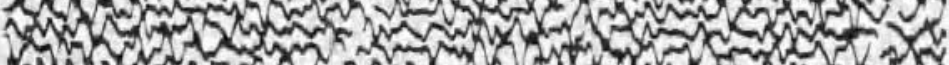

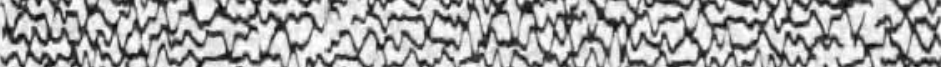

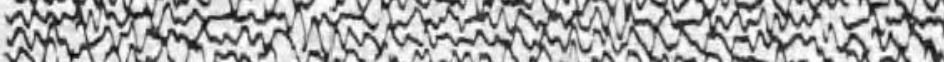
Tha

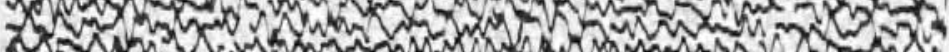

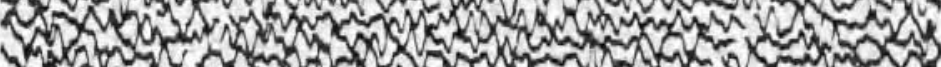

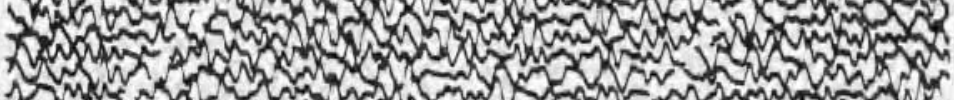

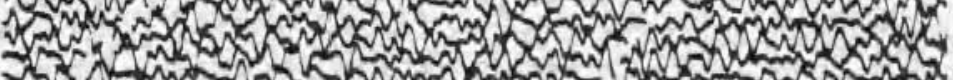

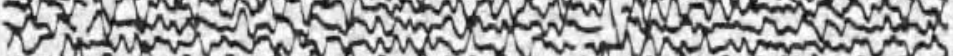

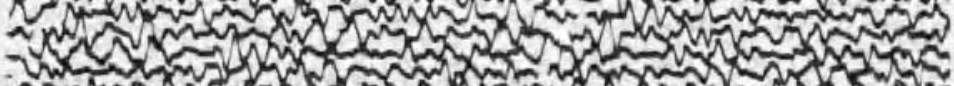

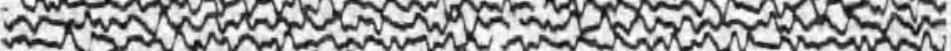

21.11

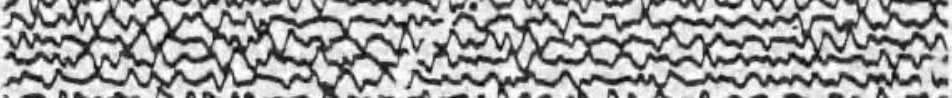
- w w n (n)

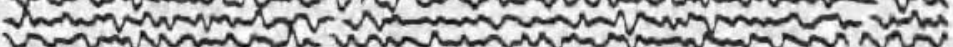

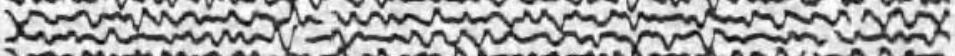
Taniman

00.11 Non, niv Han

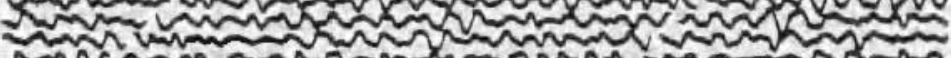
Non $32 x$ ș mon

03.11 orkn and (n) Imominar nown

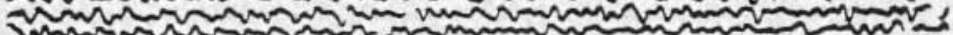
(n)

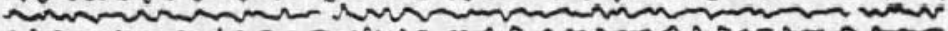
mon (N)

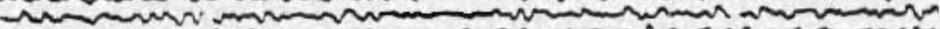
L rim ח

$08.12=20$ $16 \cdot V I I \cdot 19 ; 0$

Fig. 29 - Vedi Fig. 28.

Fig. 29 - See Fig. 28. 


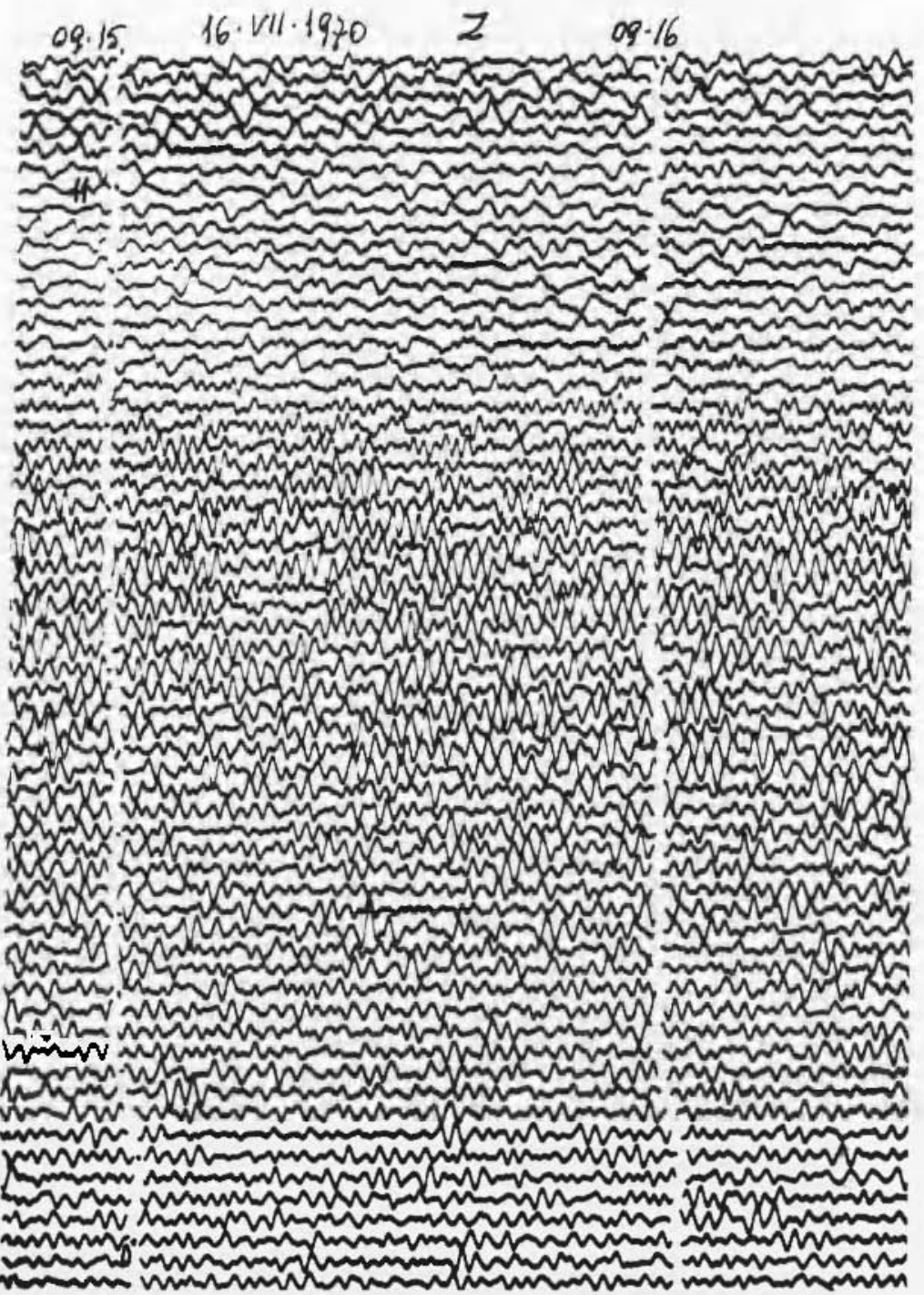

Fig. 30 - Verli Fig. 28.

Fig. 30 - See Fig. 28. 


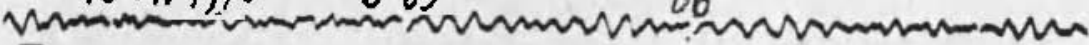

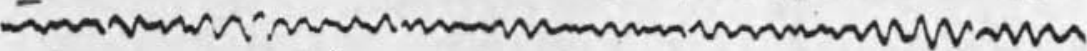

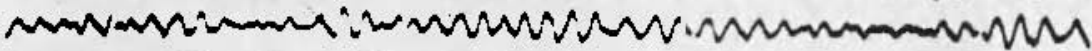

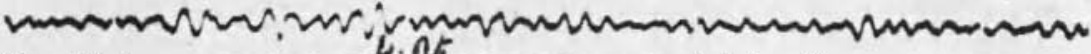

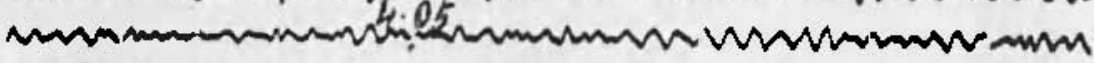

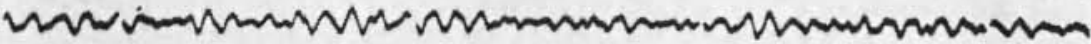

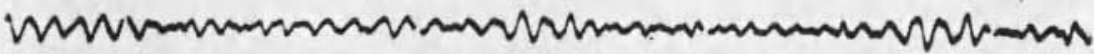

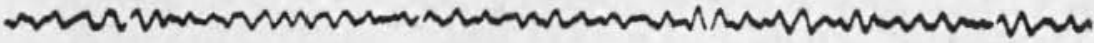

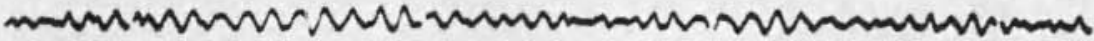

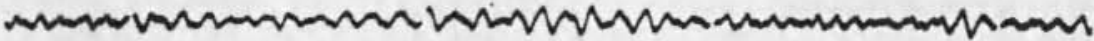

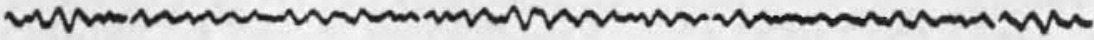

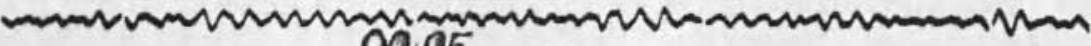

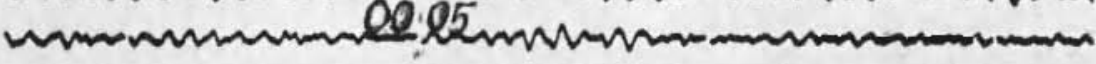

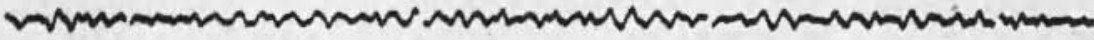

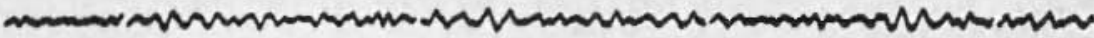

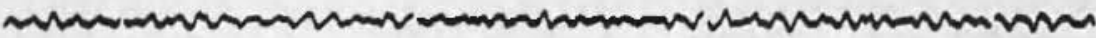

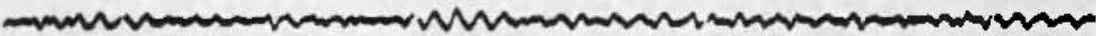

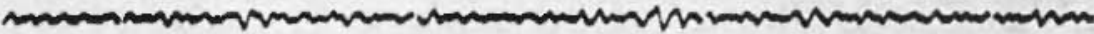
MNAMAm

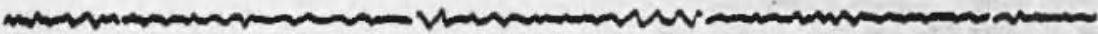

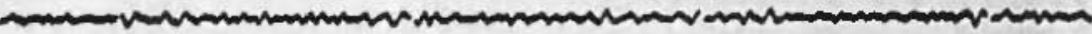

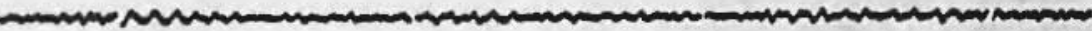
Wh" 15.711 .192018 .05 (15)

Fig. 31 - Nel suo transitare sul mar Ligure e sull'alto Tirreno, la perturba. zione del 15-16. VII.1970, provoca microsismi di ritmo svariato: quelli di minor periodo - e di minore energia - assoeiati a sistemi dlondie sporadici, temporanei, vengono registrati a Genova (figg. 28-30), dove sovrastano quelli con periodi slell'ordine di 5 sec, di maggiore energia, associati a sistemi d'ondie significativi, chiaramente registrafi a Roma (Citti Lniverkitaria), rlagli apparecchi Galitzin-Wilip ( $T o-T g-9,0$ sec ca), ivi funzionanti.

Fig. 31 During its passage over the Ligurian Sea and the High Tyrrhe. nian Sea, the perturbation of July 15 and 16,1970 brings forth microseisms of various rates: those of minor periorls and enerory are recorderl in Genoa (fig. 28-30) where they cover those with periods in the ordier of 5 sec and a higher energy loarl, associated to significant wave systems which were clearly recoried in Rome (C. U.) by the there installed Galitzin-Wilip instru ments ( $T_{0}-T g-9,0$ sec approx.). 


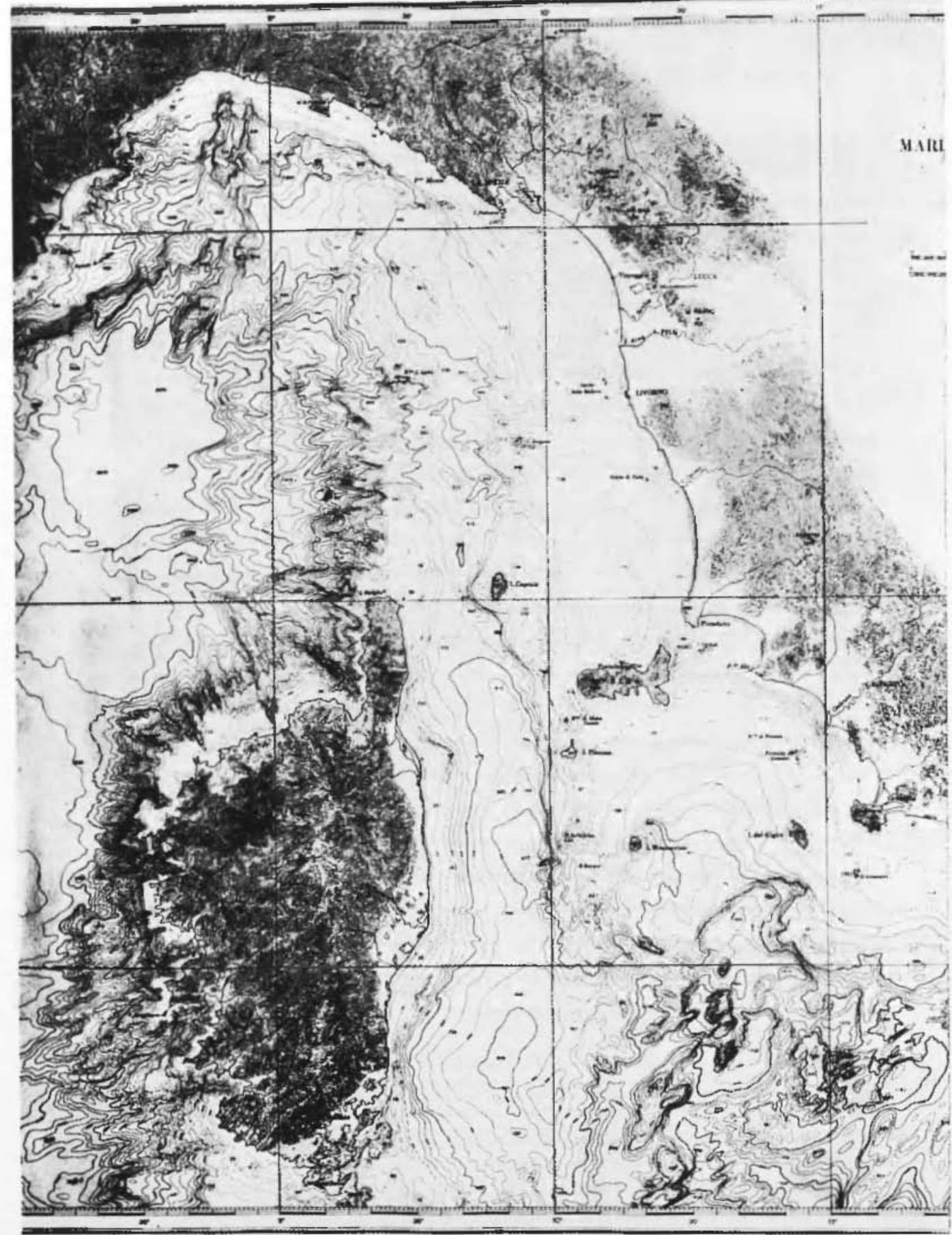

Fig. 32 La zona di mare tra La Spezia, la Capraia e l'isola d'Elba, caratterizzata da aeque relativamente poco profonde, va ritenuta come la matrice dei microsismi, con periodi dell'ordine di 5 t 1 sec, associati a sistemi d'onde significative - capaci di "sentire * il fondo -, e animati da notevole energia, che consente loro la propagazione a distanza. (Da una carta dell'Istituto Idrografico della Marina).

Fig. 32 - The sea strip between La Spezia, Capraia Island and Elba Island, with relatively limited depth8, is considered the matrix of microseisms with periods in the order of $5 \pm 1$ sec, associated to significant wave systems, able "to feel" the bottom and with a considerable energy load which allows them to propagate at a distance (from a map of the Hydrographic Institute of the Navy). 


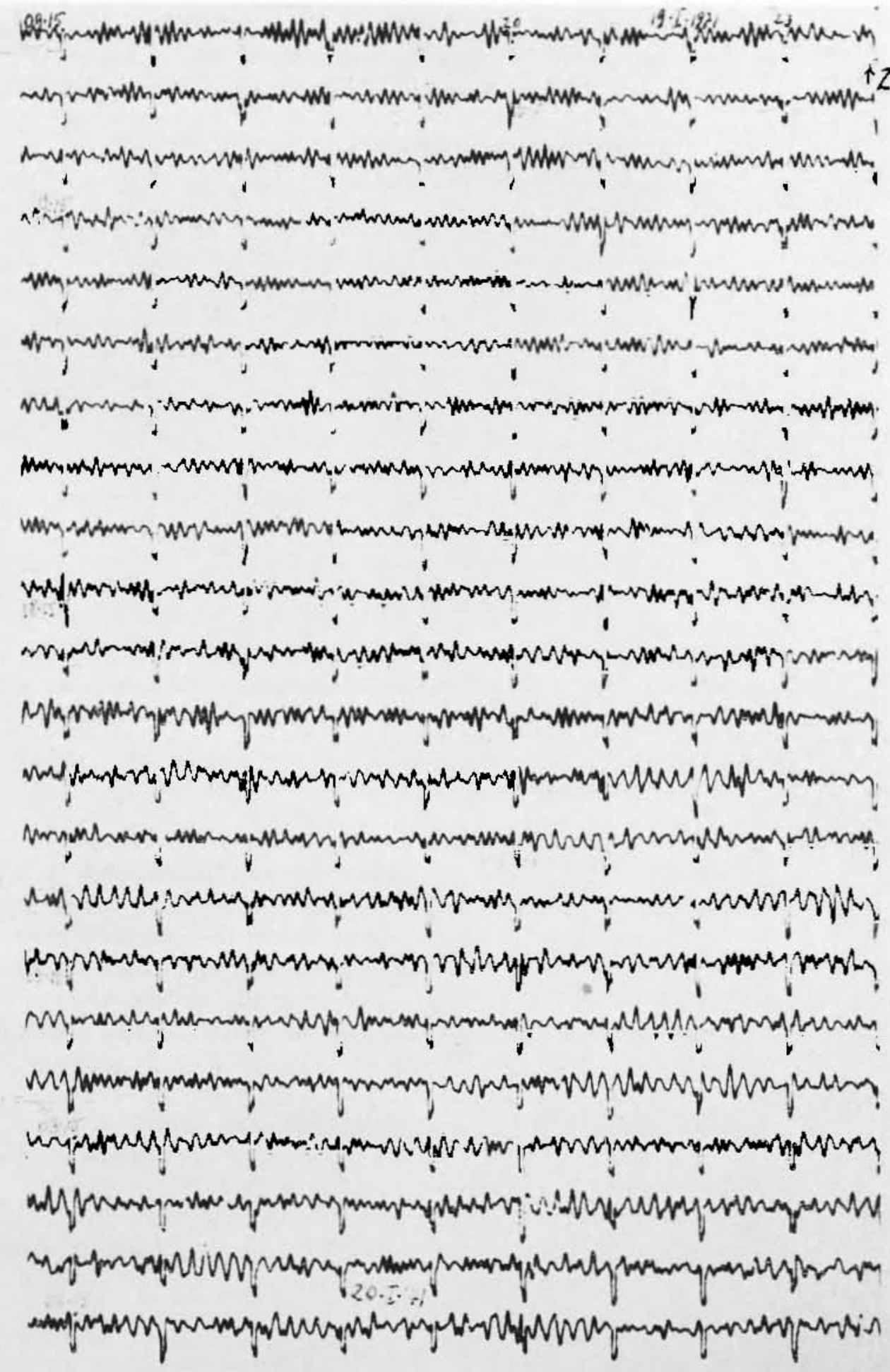

Fig. 33 - Il 19.1.1971 un'ampia zona di bassa pressione agisce nei pressi delle coste scan. dinave, provocando la creazione di microsismi, con periodi dell'ordine di $5^{\mathrm{s}, 5} \mathrm{ca}$ - caratteristici dolla zona - e nettamente registrati dalle stazioni scandinave. Dal 19 al 20 la perturbazione seandinava, unendosi ad un'altra vasta area depressionaria che dall'Atlantico procedo verso lo isole britanniche, agondo al largo delle coste occidentali di quest'ul. tima, dà origine ad una energica tempesta mierosismica, in cui prevalgono i microsismi di 8-8,5 sec, caratteristiei di questo tratto di mare. Anche a Uppsala, nella notte fra il 19 e il 20 tali microsismi cominciano a sovrapporsi a quelli d'origine scandinava $\theta$ praticamente li sostituiscono durante il 20 e il 21 (fig. 39 ), quando la vasta depressione atlantiea si approssima all'Irlanda, investendo lo isole britanniche (figg. 34, 35).

Fig. 33 - On Jan. 19, 1971 a wide area of low pressures acts near the Scandinavian coastline where it gives birth to microseisms with periods in the order of about $5,5 \mathrm{sec}$, peculiar to the area, which are clearly recorded by the Scandinavian stations. On Jan. 19 and 20 the Scandinavian perturbation combines with another wide depression area proceeding from the Atlantic Ocean toward the British Isles and acting off their West eoast, which gives rise to a strong microseismic storm in which mieroseisms of $8 \cdot 8.5 \mathrm{sec}$,peculiar to this sea strip, prevail. In the night from .Jan. 19 to 20 also in Uppsala the said microseims begin overlapping those from Seandinavia and virtually replace them during Jan. 20 and 21 (fig. 39) when the vast Atlantic depression draws near Ireland and hits the British Isles 
gli altri, i microsismi dovuti a sistemi d'onde sporalici, temporanei, vengono più o meno rapidamente assorbiti dal mezzo, tanto più rapidamente, quanto più elevata è la loro frequenza ( $\left.{ }^{4}\right)$.

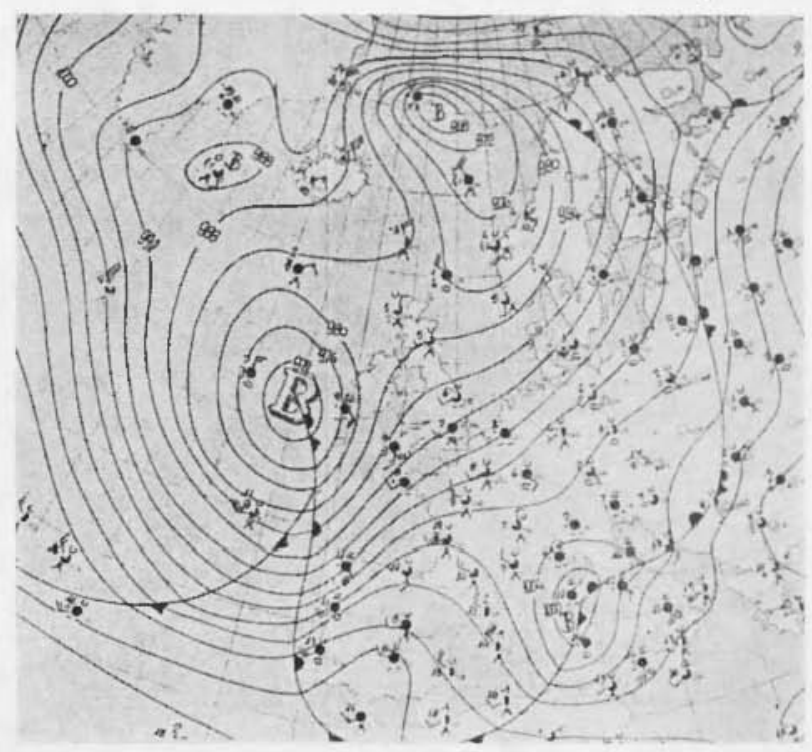

F'ig. 34 - Situazione meteorologica alle ore 12 (G.M.T.) del 20.I.1971 (dal Bollettino Meteorologico Quotidiano dell'Aeronantica Militale).

Fig. 34 - The weather situation at midday (G.M.T.) of Jan. 20, 1971 (from the Daily Meteorological Bulletin of the Air Foreo).

A differenzi di quanto avviene per i mierosismi fondamentali, che originano in mari interni, i sistemi di montagne continentali non costituiseono ostacolo sufficiente ad impedire la propagazione dei microsismi atlantici di massimo periodo: pur subendo attenuazioni diverse nelle diverse direzioni, essi vengono dovunque registrati in Europa, anche a parecchie migliaia di chilometri di distanza dalle zone-origine.

3.1. - Diamo due significativi esempi di tempeste microsismiche atlantiche.

Tra il 19 e il 20 Gennaio 1971, una depressione agisce al largo delle coste seandinave, provocando l'insorgere di mierosismi, propri dei mari di questa zona, con periodi cioè di cirea 6 sec, ampiamente 
registrati, p. es., a Cppsala (fig. 33). Nelle prime ore del 20 Gennaio, una perturbazione, dall'Atlantico settentrionale, procede verso le isole britanniche: il suo centro si trova in prossimita dell'Trlancia

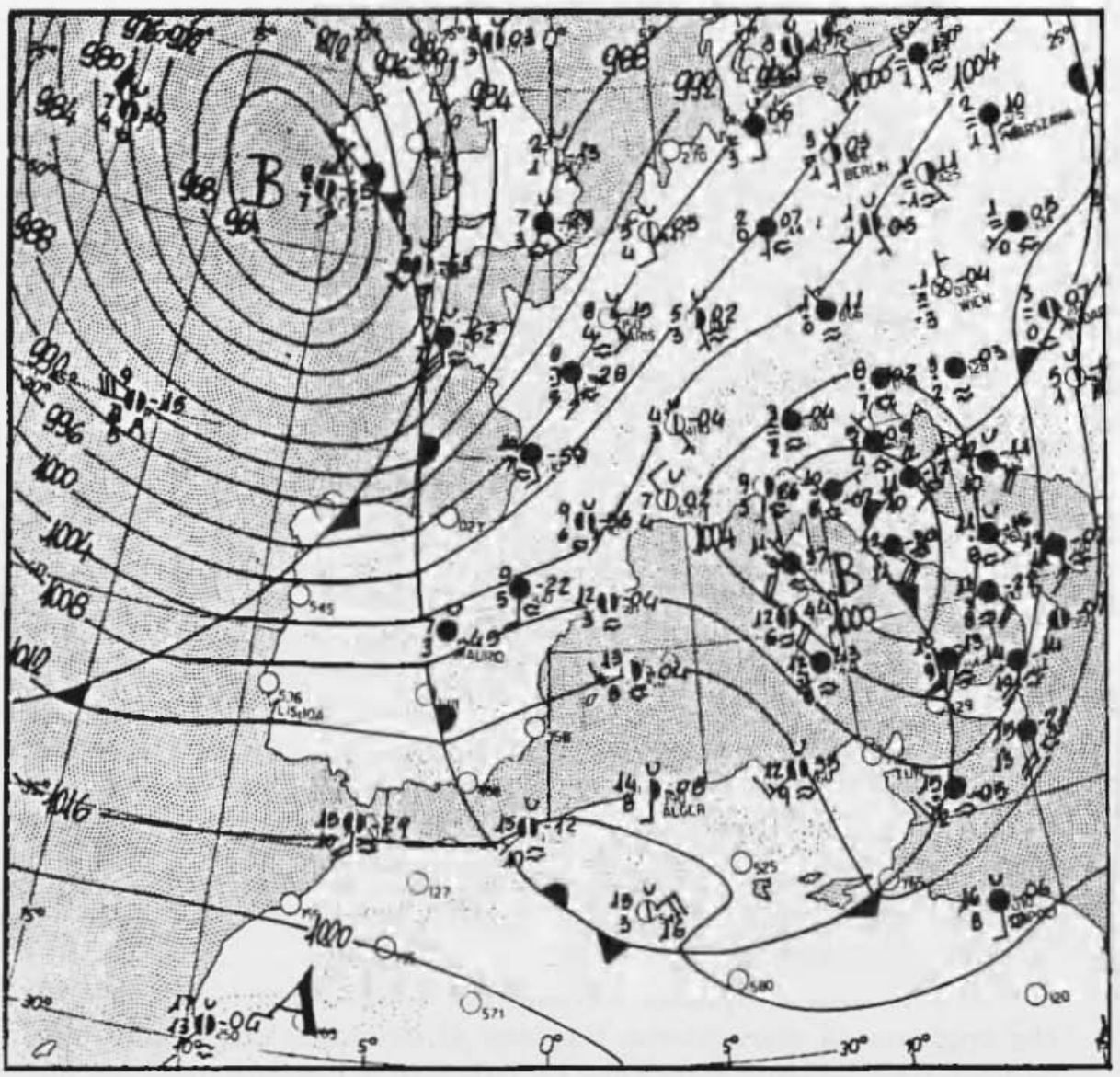

Fir. 35 - Situazione meteorologica alle ore 18 (G.MI.T.) del 20.I.1971 (dal Boll. Meteor. Quot. dell'Aeronautica Militare).

Fig. 35 - The weatler situation at $6^{\text {h }}$ p.m. (G.M.T.) of Jan. 20, 1971 (from the Daily Meteorogical Bulletin of the Air Force).

alle $12^{\mathrm{h}}$ del 20 e la raggiunge alle $18^{\mathrm{h}}$ dello stesso giorno (figg. $34-35$ ). Questa più vasta perturbazione, dà origine a sistemi di onde significative, a cui corrispondono microsismi di notevole ampiezza, con periodi di $8^{\text {a }} \mathrm{ca}$; sono questi $\mathrm{i}$ microsismi fondamentali della zona, legati 

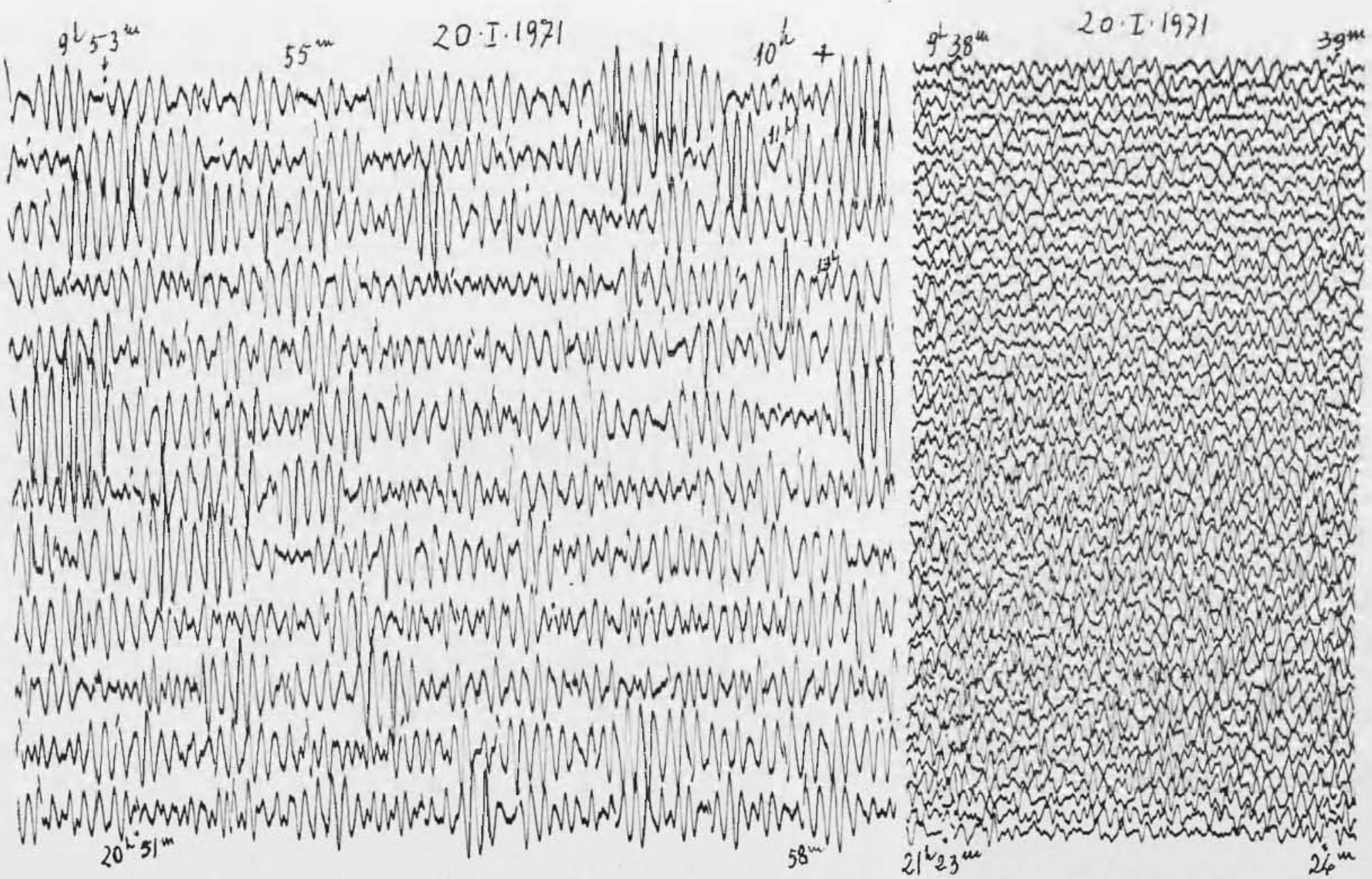

Fig. 36 - Microsismi registrati a Porto (Oporto), in occasione della perturbazione ciclonica, di cui alle figg. 34-35.

a) a sinistra della figura:

Registrazione della componente verticale "Press-Ewing" a lungo periodo (l'ingrandimento dinamico varia da 100 a 600 per periodi varianti da 1 a 8 sec): anche a Oporto, 'bull'Oceano Atlantico, i microsismi con 8 sec di periodo ca, sono nettamente predominanti, di ampiezza ancora maggiore di quella osservata a Toledo, distante dalla zona-origine circa $200 \mathrm{~km}$ in piit.

b) a destra:

Registrazione della componente verticale a breve periodo ( $T o=1 \mathrm{~s}, 0 ; T g=0,77 \mathrm{sec}$ ). Tale strumento ha un ingrandimento dinamico di oltre 40.000 per periodi di 0,6 sec e ca 1000 per periodi di 4 sec; periodi maggiori non hanno amplificazione apprezzabile. La registrazione costituisce una nuova prova dell'esistenza, lungo le coste del mare, in localita prossime alla zona-origine, di tutta una gamma di perturbazioni, legate al mare agitato da moti ondosi temporanei, sporadici, su cadenze svariatissime: perturbazioni di piccola ampiezza, di carattere esclusivamente locale, rapidamente assorbite dal mezzo. I microsismi persistenti, di massima ampiezza, sono quelli associati ai sistemi d'onde a significativen: nel caso specifico, quelli propagantisi, sul ritmo di 8 sec ca, dalla zona-origine a Oporto, a Toledo, a Uppsala, a Roma ed oltre, su tragitti di migliaia di km.

Fig. 36 - Microseisms recorded at Oporto (Portugal) during the cyclonic perturbation indicated in figs. 34 and 35. a) on the left of the figure:

Recording of the vertical "Press-Ewing" component having a long period (the dynamic magnification varies from 100 to 600 for periods between 1 and $8 \mathrm{sec}$ ); also in Oporto, on the Atlantic shore, the $8 \mathrm{sec}$ microseisms prevail without doubt. Their amplitude is still larger than that observed at Toledo, some $200 \mathrm{kms}$ more distant from the origin-area.

b) on the rigt:

Recording of the vertical component of a short period ( $T o=1 s, 0 ; T g=0,77 \mathrm{sec})$. The instrument has a dynamic magnification of over 40.000 for periods of $0,6 \mathrm{sec}$ and of about 1000 for periods of $4 \mathrm{sec}$; larger periods have no appreciable amplification. The recording gives another evidence of the existence, along the coastline, near the origin-area, of an entire range of perturbations connected to the rough sea by sporadic, temporary wavy movements at highly variable rates: perturbations of small amplitudes, of an ex. clusively local character, which are rapidly absorbed by the medium. The persistent microseisms of maximum amplitude are those associated to the "significant" wave systems, in this specific case the microseisms at the rate of abont 8 sec from the origin-area toward Oporto, Toledo, Uppsala, Rome and beyond, covering distances of thousands of $\mathrm{kms}$. 


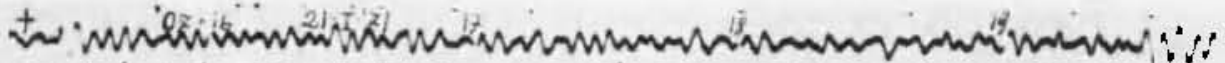

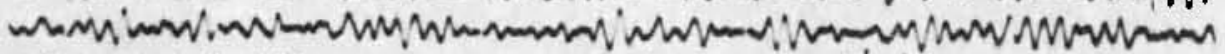

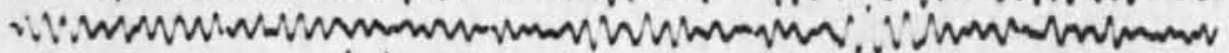

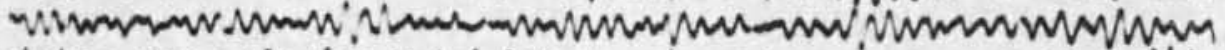

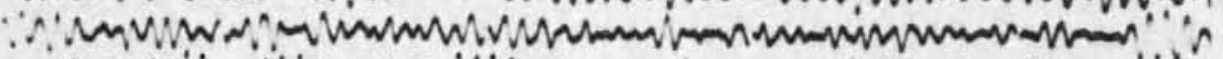

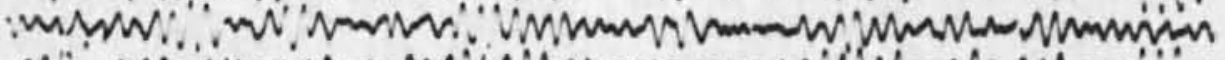

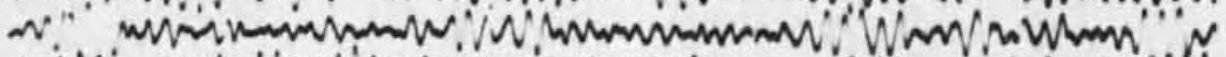

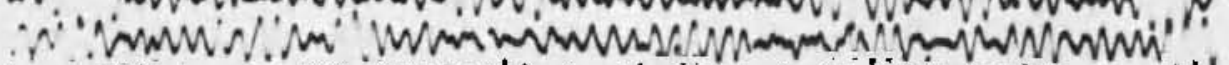

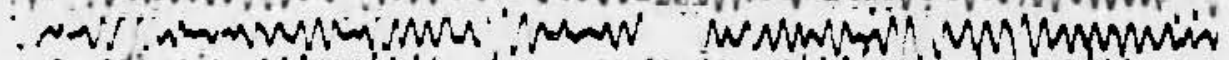

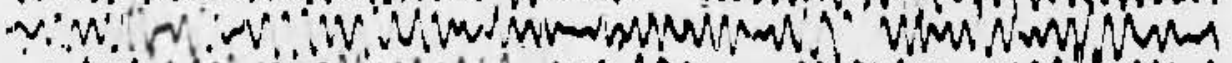

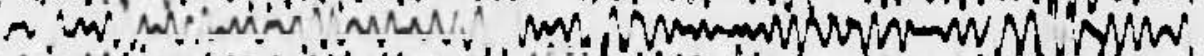

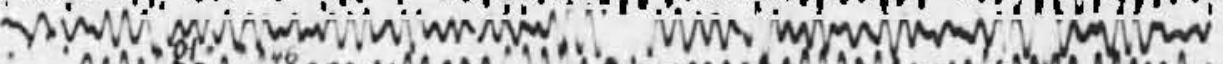

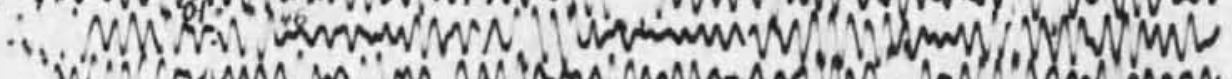

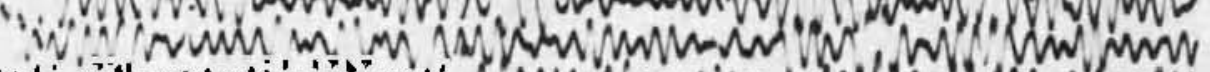

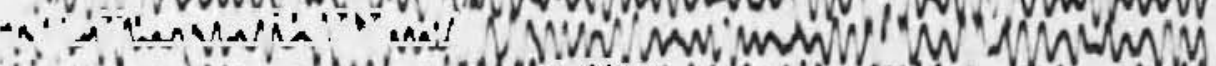

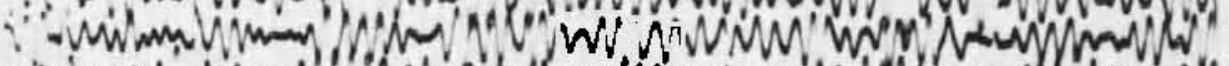

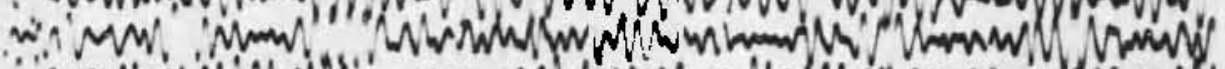

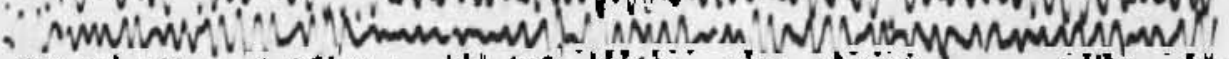

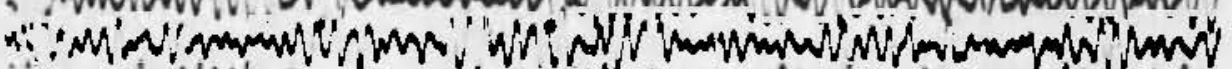

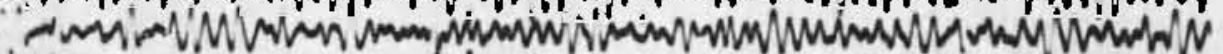

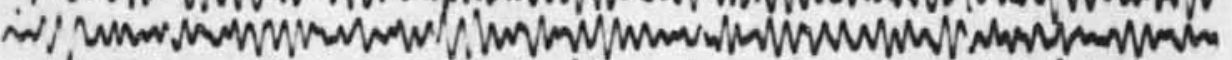

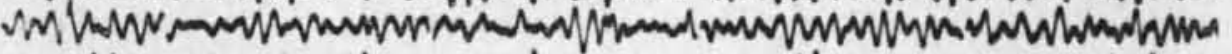

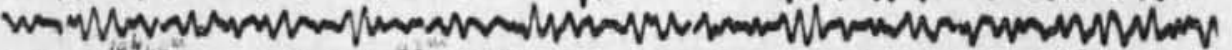

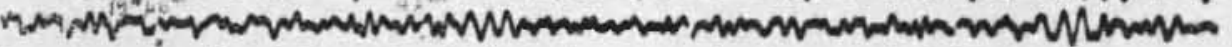

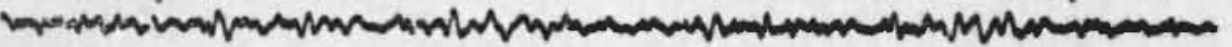

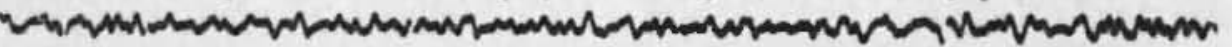

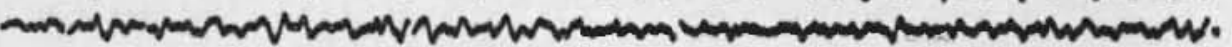
manch No

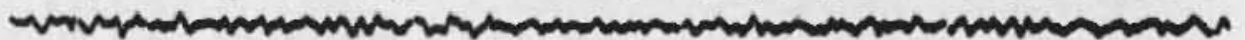
1

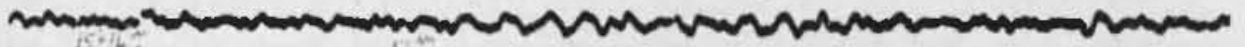

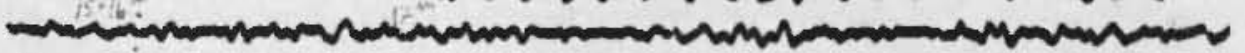

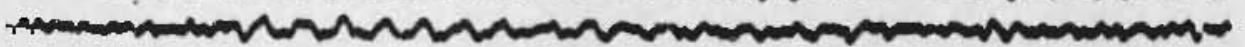

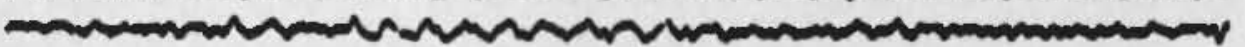
mimar

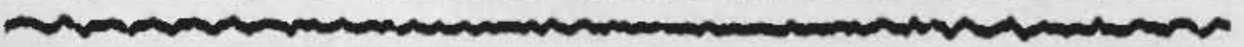

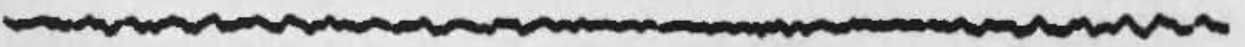

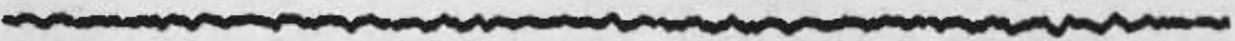
monom

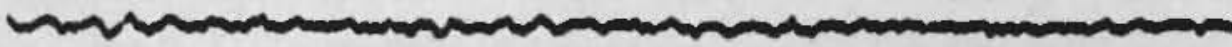
WhNon in

Fig. 37 - Componente verticale "Wilip " di Roma, Citta Universitaria (tempo solare me dio). I microsismi con $8^{\mathrm{s}} \mathrm{ca}$ di periodo, di provenienza nord-atlantica (figg. 34-35), verso le $10^{\mathrm{h}}$ ea del 20 Gennaio sono ancora poco perturbati (in basso, nella figura). $A$ mano a mano clie la depressione mediterranea formatasi verso le $12^{\mathrm{h}}$ del 20 Gennaio (fig. 34) dal grolfo di Genova procede verso l'alto Tirreno, comincia la registrazione di microsismi di $4^{\text {s }}$ ca, che si sovrappongono a quelli di origine atlantica e finiscono per mascherarli completamente durante il transito della perturbazione sul medio Tirreno. Esempio di contemporanea registrazione di mierosismi, provenienti da diversa origine: quelli di sorgente più prossima alla stazione d'osservazione, anche se originariamente di ampiezza nettamente inferiore, prevalgono fino ad occultare gli altri, di origine ben più lontana.

Fig. 37 - Vertical "Wilip" component of Rome - C. U. (Mean Solar Time). Microseisms of a period of $8 \mathrm{sec}$ approximately, from the North Atlantic area (fig. 34 and 35), still show little perturbation towards $10^{\mathrm{h}} \mathrm{a} . \mathrm{m}$. of Jan. 20 (see lower part ot figure). As the Mediterranean depression, built up towards midlay of Jan. 20 (fig. 34), shifts from the Gulf of Genoa toward the High Tyrrehnian Sea, the recording of microseisms of about 4 ser begins. These overlap those from the Atlantic and at last cover them entirely when the perturbation passes over the Tyrrhenian Sea. An example of simultaneous re. cording of microseisms from various origins: those rising nearer to the observation station, even if their amplitude is originally elearly lower, prevail and at last cover the others of much farther origin. 


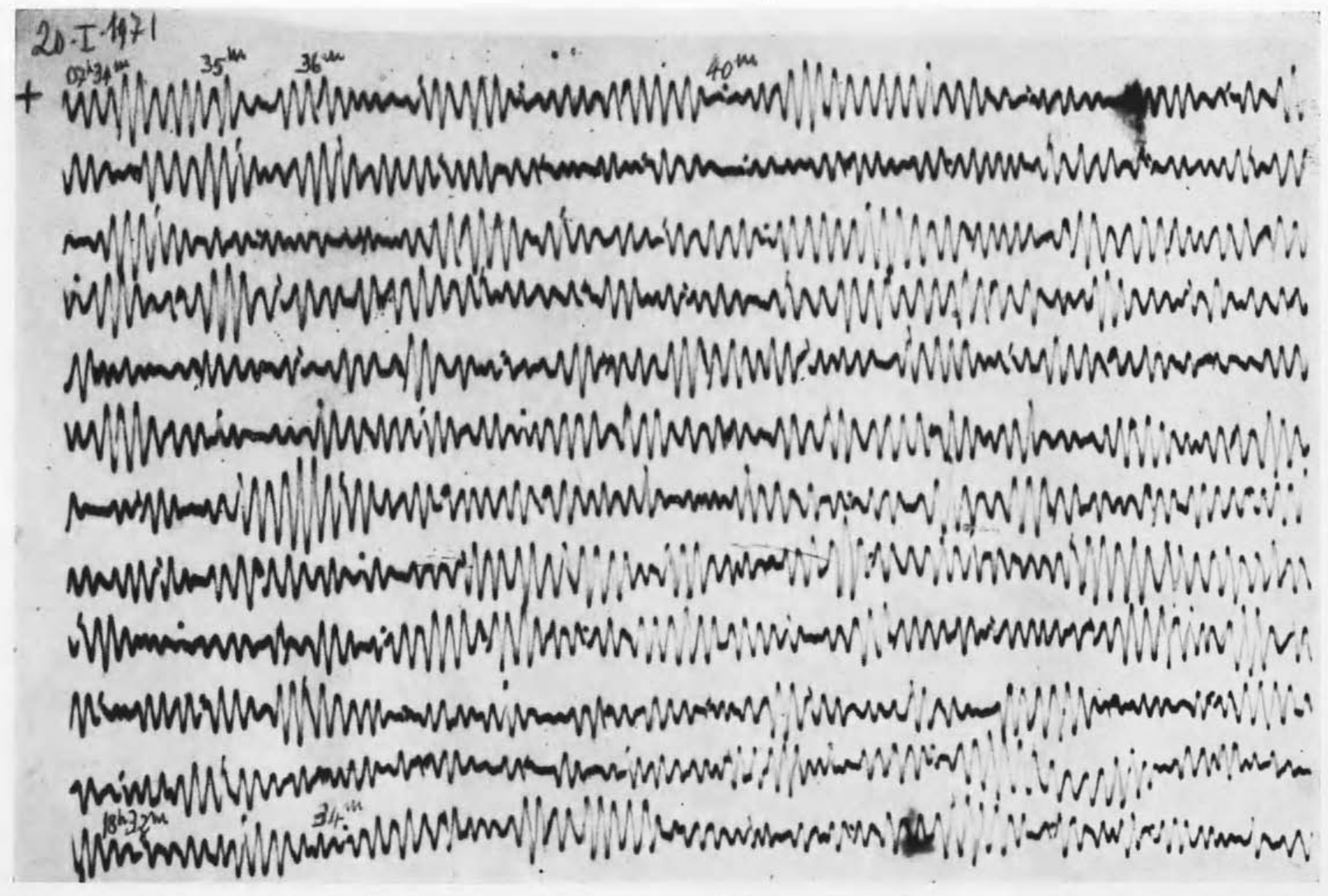

Fig. 38 - Componente verticale "Press-Ewing ", a lungo periodo, di Tolerlo (T.MI.G.). I sismografi a lungo periodo lli Toleilo, durante i giorni 20 e 21 Gennaio 1971, registrano quasi esclusivamente, $\theta$ con grande ampiezza, i microsismi con periodo di $8^{\text {s }}$ ca $(8.89,5)$, provenienti dalla grante perturbazione ciclonica atlantica, di cui alle figg. 34-35. Mentre a Roma il transito della perturbazione tirrenica provoca microsismi tli $4^{\mathrm{s}} \mathrm{ea} \mathrm{(fig.} \mathrm{37)} \mathrm{ehe} \mathrm{coprono,} \mathrm{data} \mathrm{la} \mathrm{vicinanza} \mathrm{della} \mathrm{zona-origine,} \mathrm{i} \mathrm{microsismi}$ di provenienza atlantica (elıe originano a quasi $2000 \mathrm{~km}$ da Roma), a Toledo (che si trova a llistanza pressoché uguale dai due centri di perturbazione) non v'è traccia dei microsismi di origine tirrenica. Ciò s̀ soprattutto dovuto all'energia dell'interazione aria-acqua della perturbazione ciclonica atlantica, enormemente maggiore di quella tirreniea, e, in parte, al fatto che la prima agisce su una eadenza fondamentale rli periorlo pressoché doppio di quella caratteristica della seconda.

Fig. 38 - Vertical long period "Press-Ewing" component of Toledo (G.MI.T.). The long period seismographs installed in Toledo record on Jan. 20, and 211971 almost exchsively and with high amplitules the mieroseisms of about 8 second periorls (88.8.,5) deriving from the large Atlantic cyclonie perturbation indicated in figures 34 and 35 . While in Rome the passage of the Tyrrhenian perturbation brings forth microseisms of about $4 \mathrm{sec}$ (fig. 37) which cover those from the Atlantie originating at almost $2000 \mathrm{kms}$ from Rome, since the former are near the origin-area, no trace of microseisms of Tyrrhenian origin was measured in Toledo which is about the same distance from the two perturbation centres. This is cliefly due to the ellergy of the air-water interaction of the Atlantic cyclonic perturbation which is far higher than that of the Tyrrhenian Sea, as well as to the fact, at least partially, that the former acts at a fundamental period rate which is about twice the rate peculiar to the latter. 
o.

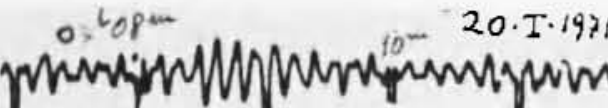

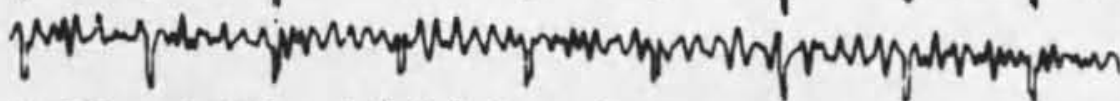

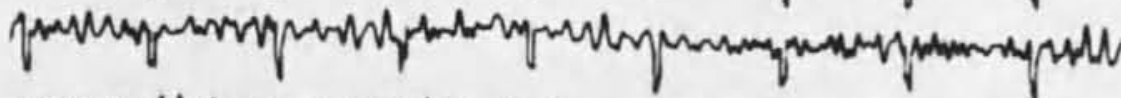

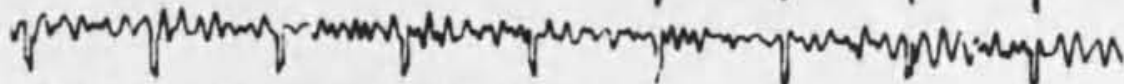

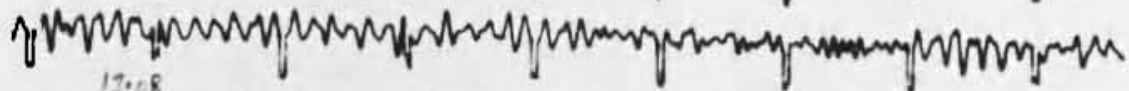

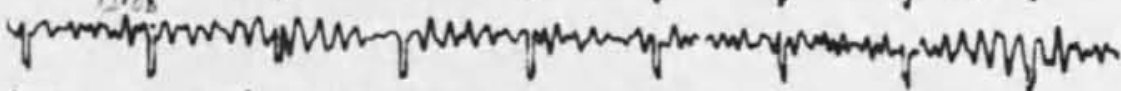

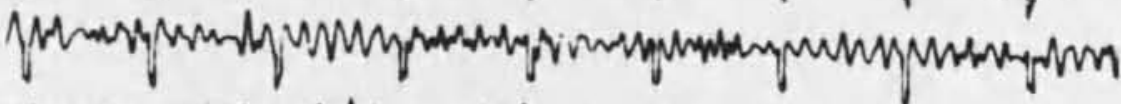

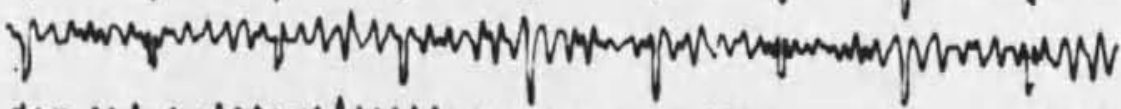

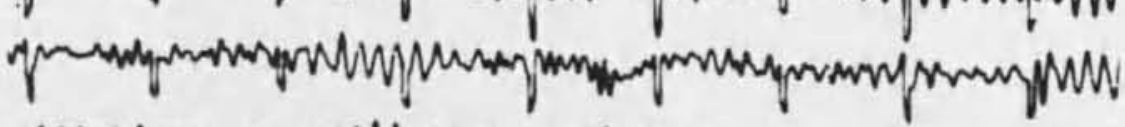

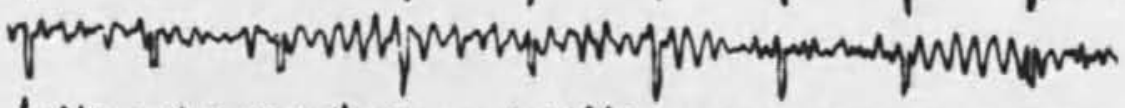

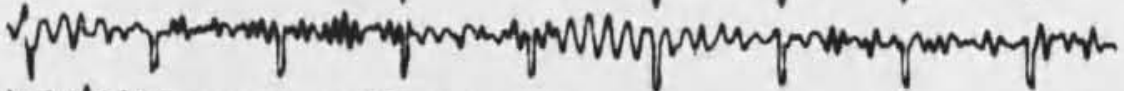

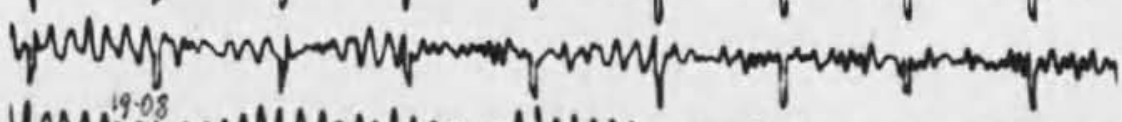

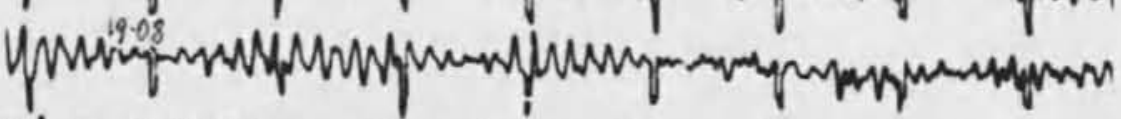

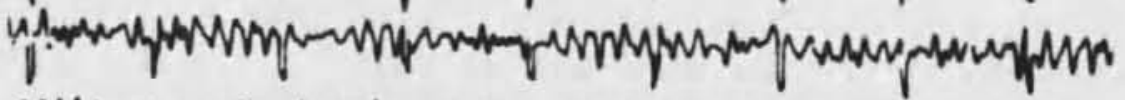

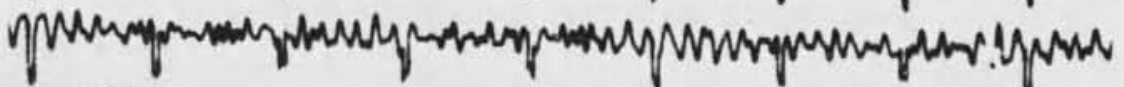

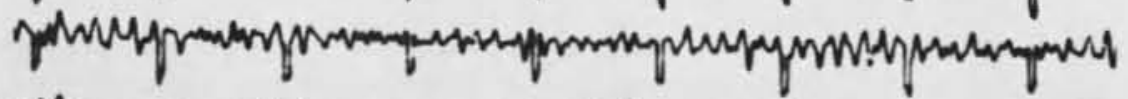

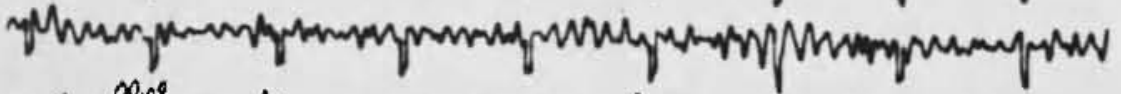
panoviog

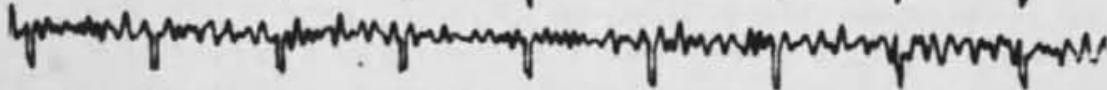
qrin.m.

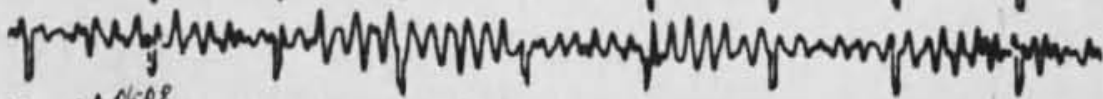

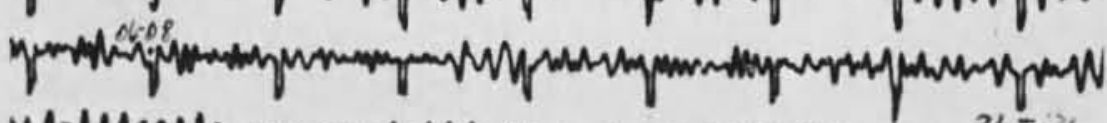

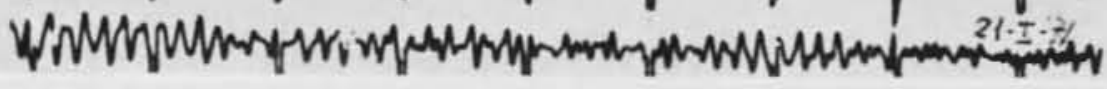

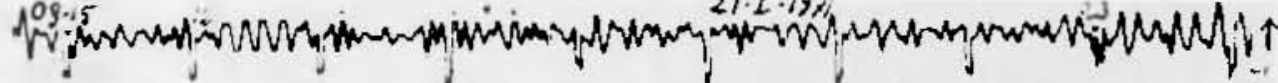

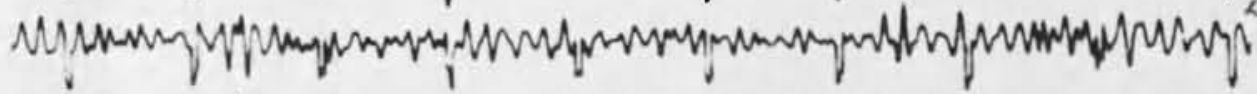

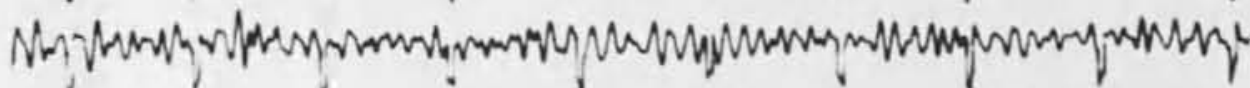

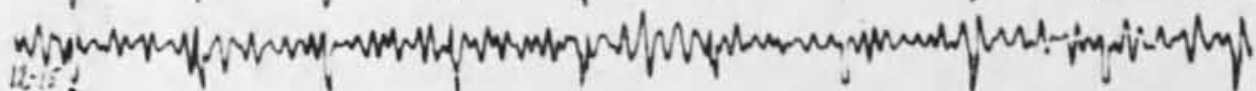

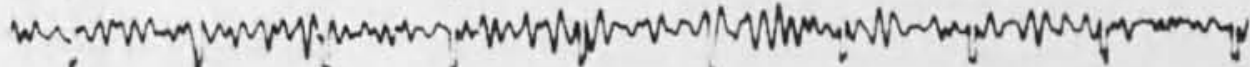

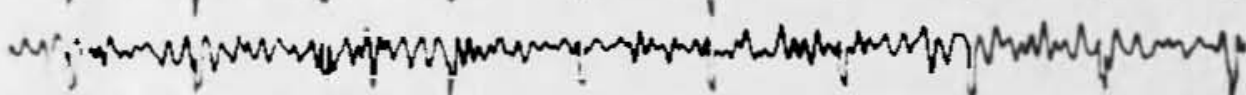

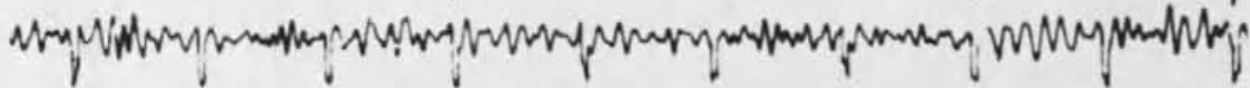

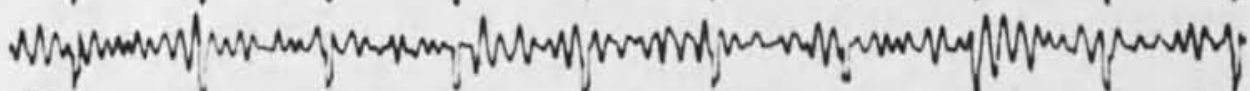

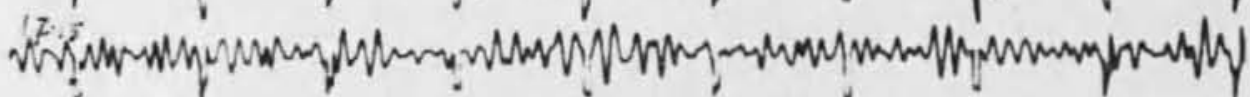

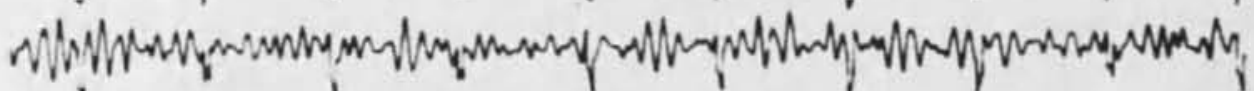

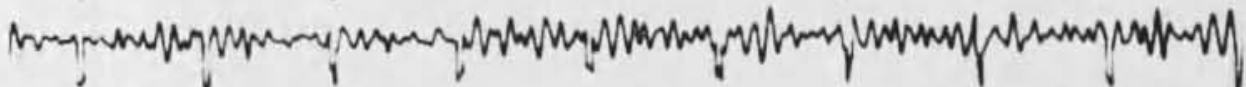

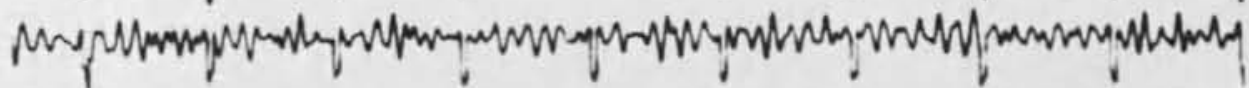

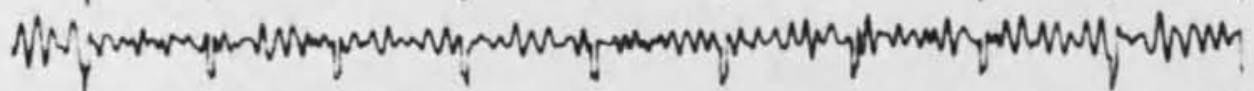

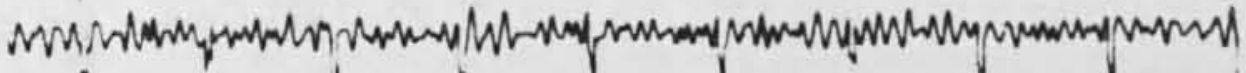

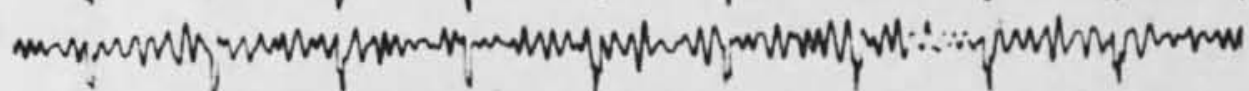

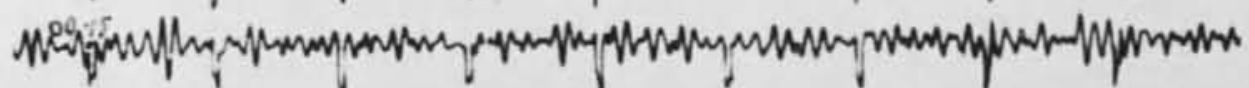

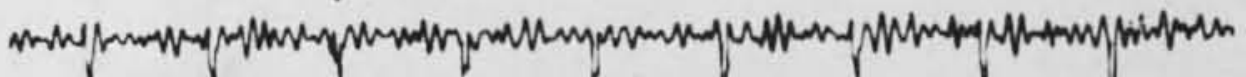

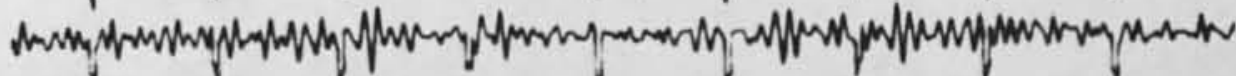

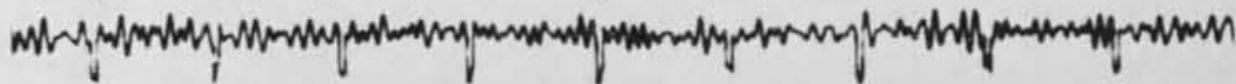

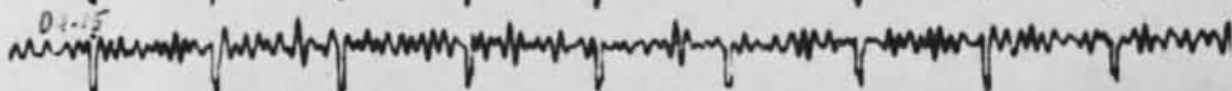

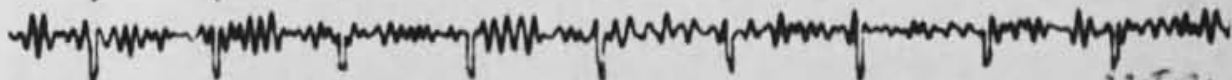

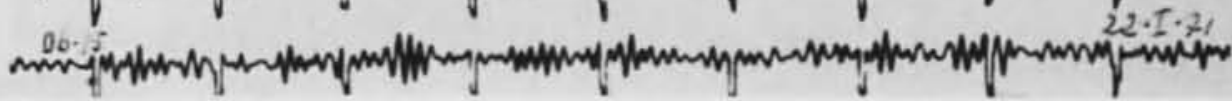

Figr. 396

Fig. $39 a$ e $39 b$ - Regristrazione di microsismi della componente verticale Press-Ewing di Uppsala, assoriali alle perturbazioni di rui allo figg. 34-35. Prevalgono nettamente i mierosismi di 8-8,5 sec, propri della fascia atlantica ad ovest dell' trlanila. Ad iniziare col giorno 22.1 , $1971 \mathrm{col}$ passaggio della perturbazione nel mare del Nord, a Uppsala i microsismi di 5 , 5 sec ca - propri della fascia ti mare aul uvest della Scundinavia -, riprendono il sopravvento (tig. 39b).

Fig. $39 a$ and $39 b$ - Recording of microseisins of the vertical "Press.Ewing" component of Uppsala, astociated to the periurbation shown in figures 34 and 35 . The microseisms of $8-8,5$ sec, peculiar to the Atlantic strip West of lrelanil, are clearly provailing. Be ginning with Jan. 22, 1971 the microseisms of about $5,5 \mathrm{sec}$, peculiar to the sea strip West of Seandinavia, dominate at Uppala when the perturbation passes over the North Sea (fig. $39 b$ ). 
a sistemi di onde significative, che hanno raggiunto il loro pieno sviluppo (4). Essi vengono registrati, con notevole ampiezza, in tiutte le stazioni sismiche munite di sismografi a medio e lungo periodo: a Porto (fig. 36), a Toledo (fig. 38), a Roma (fig. 37), ecc. Anche

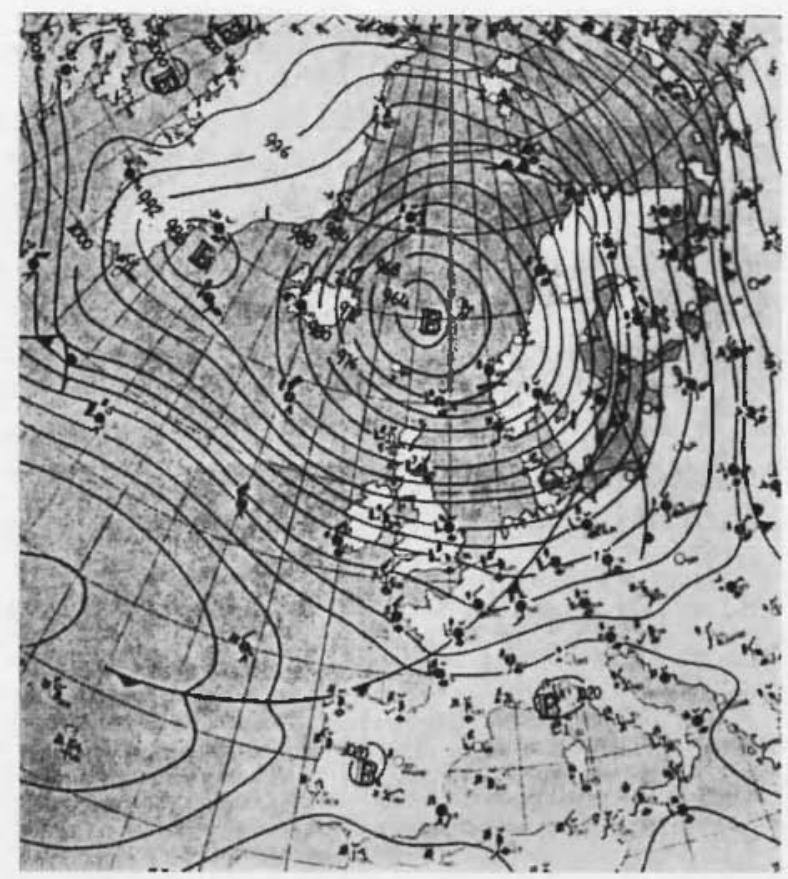

Fig. 40 - Situazione meteorologrica sull $A$ tlantico nord-orientale e sull'En. ropa alle $12^{\text {h }}$ del 13 Febbraio 1971 ("Boll. MLeteor. Quot. " Servizio Meteor. Aeron. Mil.).

Fir. 40 - The weather situation for the North-East Atlantic area and for Europe at midday of Febr. 13, 1971 (front the Taily Meteorological Bulletin of the Weather Service of the Air Force).

nelle stazioni sismiche scandinave, la tempesta microsismica locale, legata alla perturbazione piì settentrionale, viene gradatamente sopraftatta da quella associata alla perturbazione agente al largo delle isole britanniche (p. es., a Uppsala, fig. 39); e ciò per tutta la durata del 21 Gennaio. Nel frattempo, il formarsi di una perturbazione nell'alto Tirreno, provoca una tempesta microsismica locale, che a Roma (C. U.) si sovrappone a quella atlantica (fig. 37). 
Un'altra notevole tempesta microsismica atlantica ì stata quella verificatasi verso la meta di Febbraio 1971. Alle $12^{\text {n }}$ del 13 Febbraio, una vasta area ciclonica si stende fra l'Islanda e la Norvegił (fig. 40). I’attività microsismica si presenta mista per la Seandinavia: a Uppsala, infatti, si altermano i gruppi di oscillazioni con periodi dell'ordine di $6 \mathrm{~s}$, proprio dei microsismi originanti nella fascia di mare prospiciente la Scandinavia, con i gruppi đi oseillazioni con periodo dell'ordine di 8 , proprio dei microsismi della zona atlantica al largo delle isole britanniche; questi ultimi, sensibilmente più ampî̀ (fig. 41). Alle maggiori distanze, però - come di consueto -, ginugono solo $\mathrm{i}$ microsismi con periodo di $8^{\mathrm{s}}$ ca (Roma C. C., fig. 43). Con l'evolversi clella situazione meteorologica, l'area ciclonica si sposta verso $\mathrm{SW}$, investenclo progressivamente ed esclusivamente le isole britanniche (figg. 44,45). Contemporaneamente, a Uppsala si attenna via via l'agitazione microsismica di carattere "scandinavo", mentre si accentua vieppiì quella di origine prettamente atlantica, che alla fine prevale in forma pressochè esclusiva (fig. 42).

Per i motivi piu volte ardotti, anche le stazioni sismiche scandinave (Uppsala, Umea, ...) a breve periodo proprio, non registrano i microsismi con periodi superiori a 4-5 sec. Gli strumenti a breve periorlo proprio, come altrove, in caso di grosse tempeste microsismiche, registrano impulsi disorcinati, in eni prevalgono periodi fra 2 e i 3 see, propri di perturbazioni locali (Mar Baltico, Golfo di Botnia).

\section{Conciusioni.}

a) Vione sottolineato l'eccezionale interesse di microsismi provenienti dal piccolo lago artificiale di Pieve di Cadore e provocati dall'interazione fra le acque del lago e le fluttuazioni della pressione atmosferica, in fase positiva o no, transitanti sul lago. Ise fluttuazioni attive possono essere di trascurabile entità (frazioni di Torr.); esse sono particolarmente attive se associate a pressione crescente.

Tali microsismi hanno periodi generalmente inferiori al secondo e si estinguono molto rapirlamente: sovente insorgono contemporaneamente (o addirittura precedono) microsismi a più lungo periodo, fino a $4-\bar{o}$ sec. 


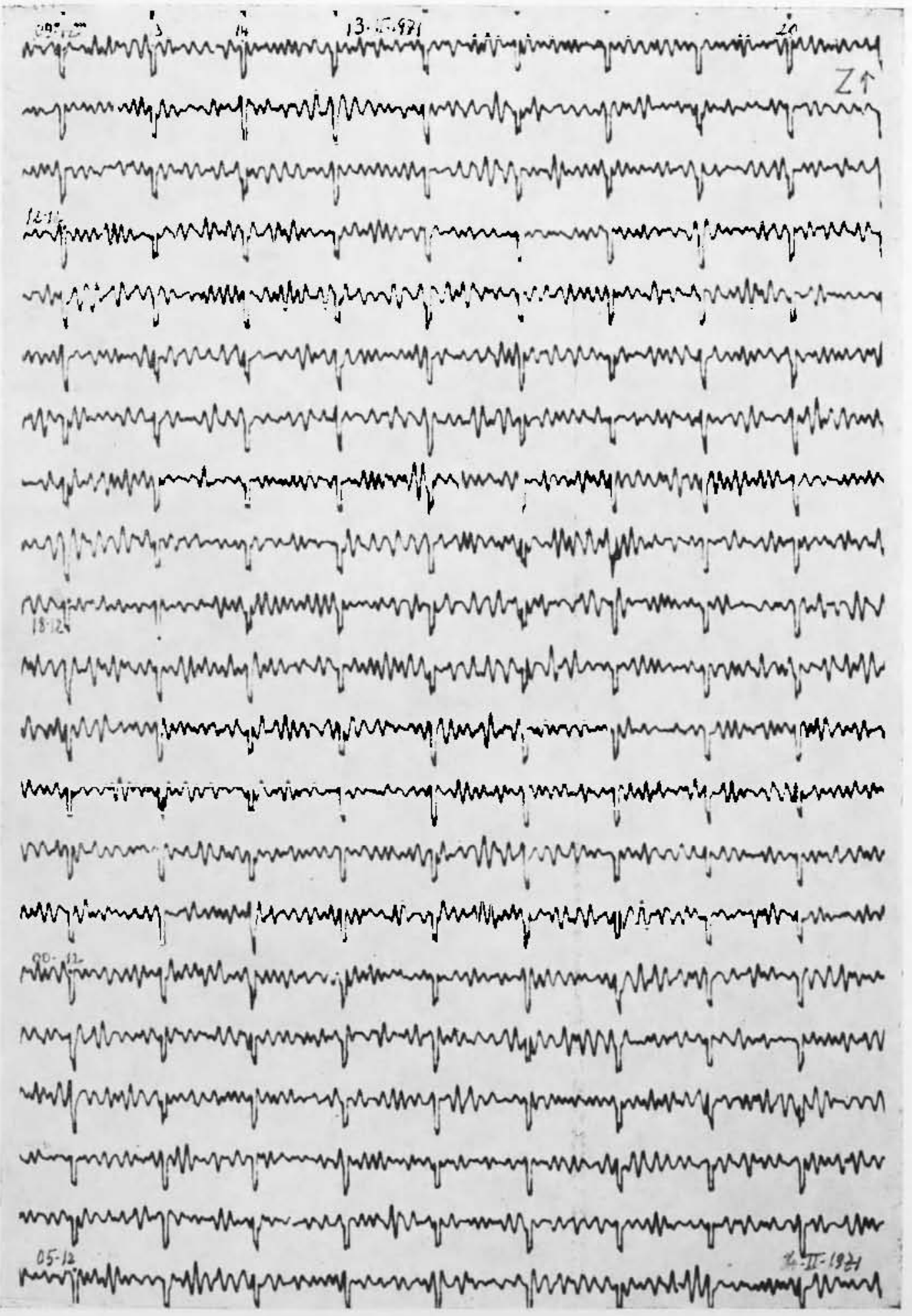

Fig. 41 - La vasta perturbazione, di cui alla fig. 40, agisce contemporaneamente nolle zono di mare presso le coste scandinave e presso le coste occidentali delle isole britanniche: di qui l'alternarsi dei gruppi di microsismi, con periodi dell'ordine di 5,5 sec e $8-8,5$ sec, propri della prima e della seconda zona, rispettivamente. Con lo spostamente verso SW della zona perturbante ed il successivo investimento della zona di mare ad ovest dell'Irlanda (figg. 44.45) i microsismi di 8-8,5 sec ca a Uppsala prevalgono nettamente, se non esclusivamente (fig. 42).

Fig. 41 - The wide porturbation shown in fig. 40 acts simultaneously in the sea area near the Scandinavian coast and near the Wost coast of the British Isles, which explains the alternation of groups of microseisms with periods in the order of $5,5 \mathrm{sec}$ and $8-8,5$ sec, peculiar to the former and to the latter area, respectively. When the disturbance area shifted toward $\mathrm{SW}$ and affected the sea strip West of Iroland (figures 44 and 45 ), the $8 \cdot 8,5$ sec microseisms prevail clearly, if not exclusively, at Uppsala (fig. 42). 


$$
\text { Upsala 14.II.1971 z }
$$

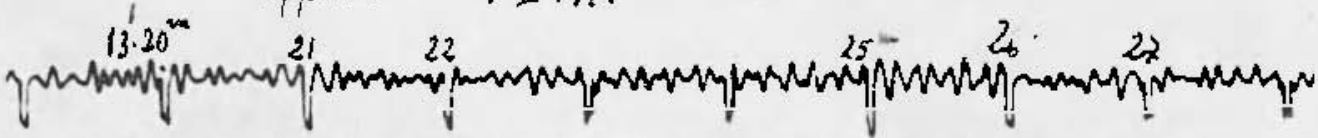

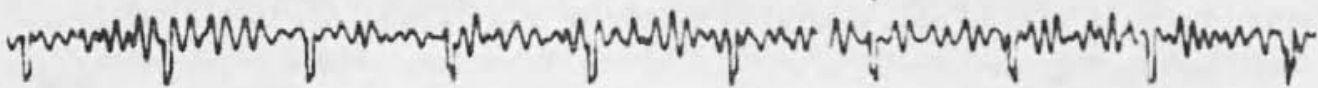

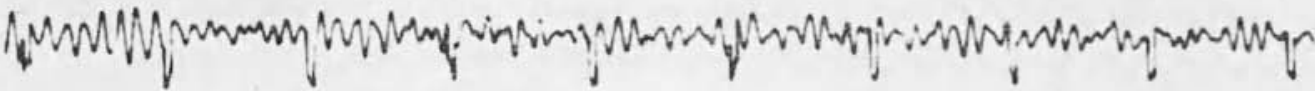

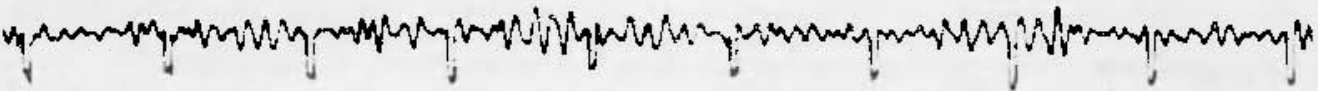

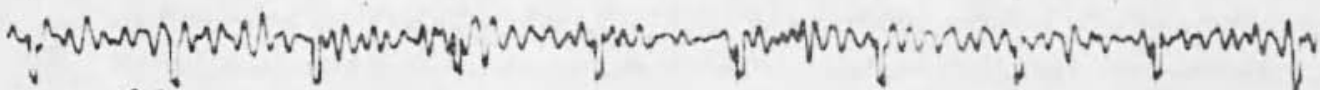

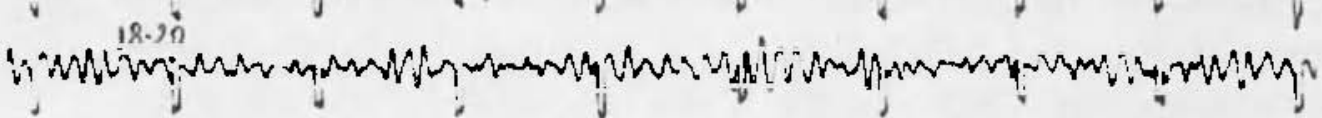

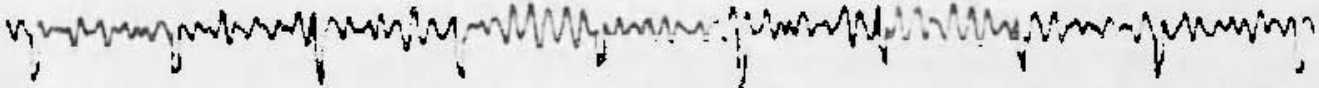

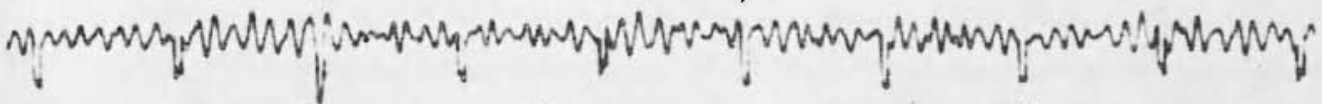

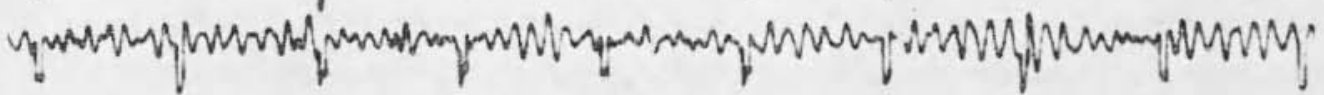

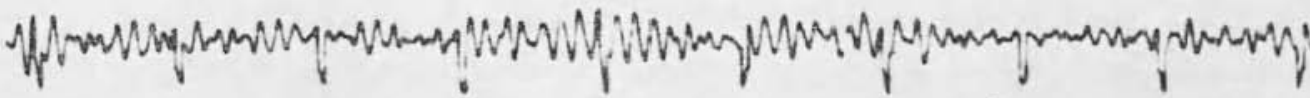

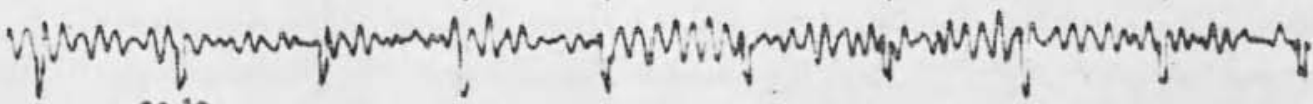

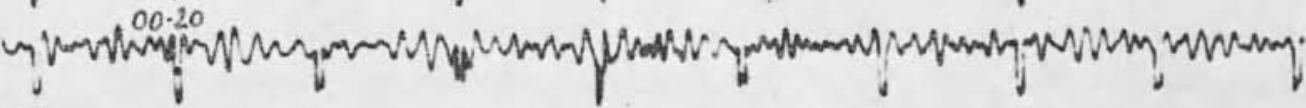

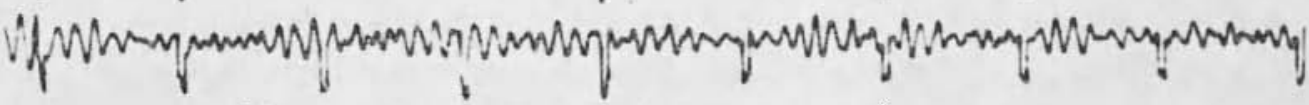

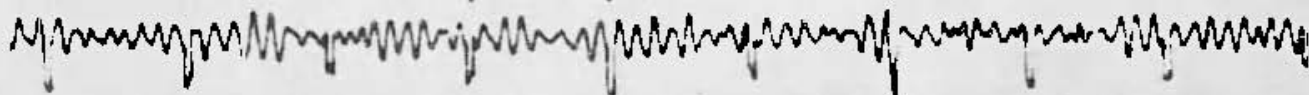

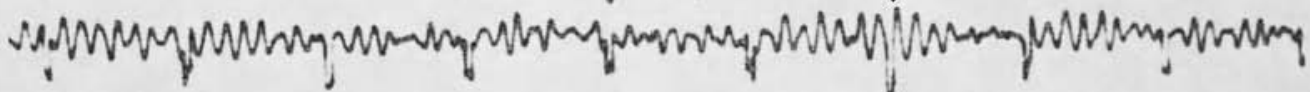

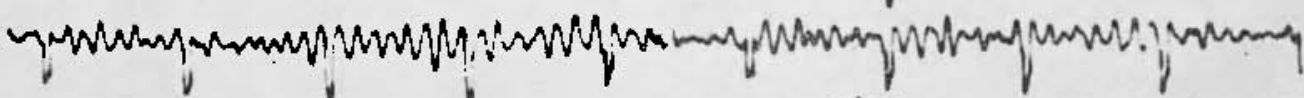

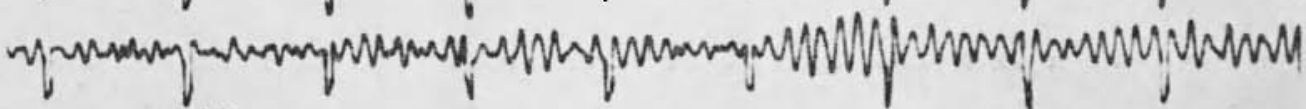

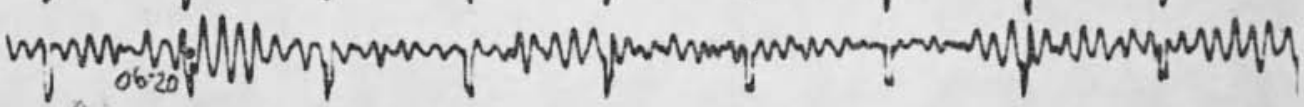

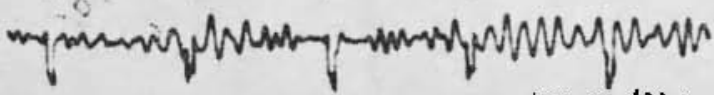

$$
\text { 15. II. } 1921
$$




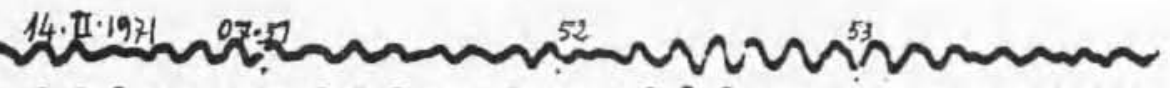
"Non

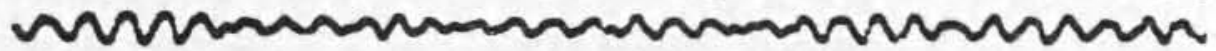
Mamm

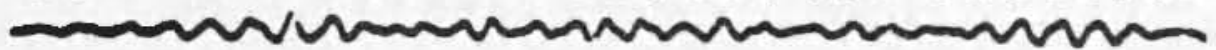

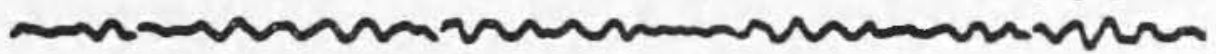
Mnmm

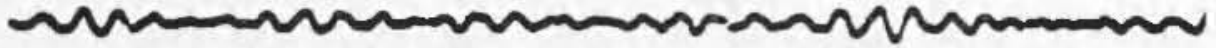

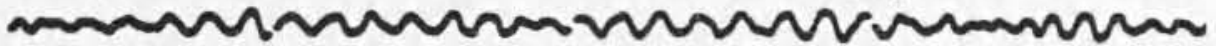

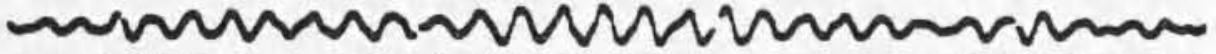
m

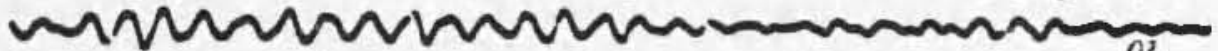
ONONOMn

Whann Nannmannmon

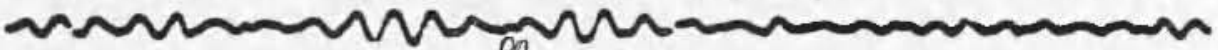

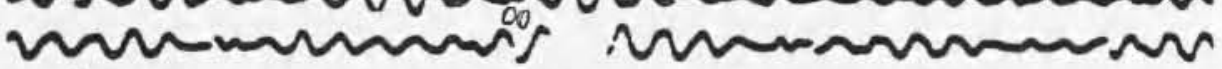

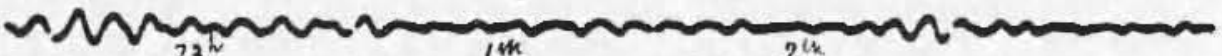

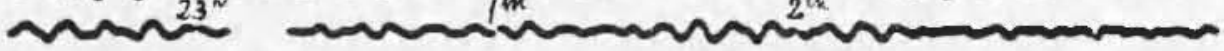

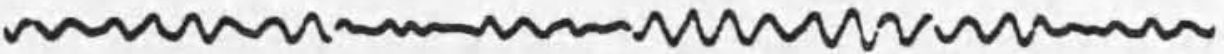

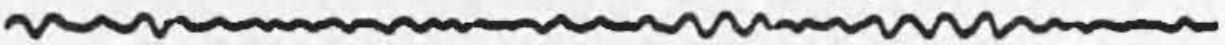

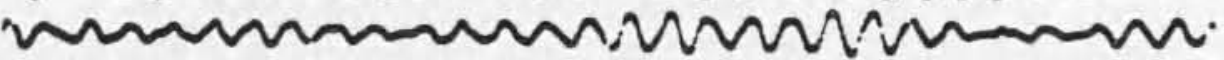

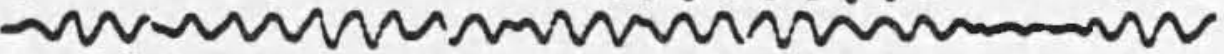

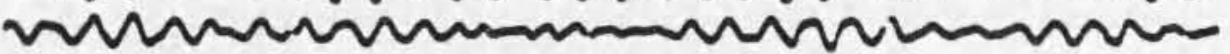

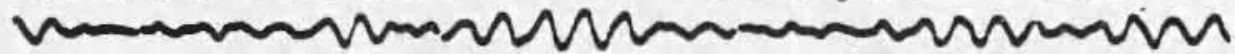
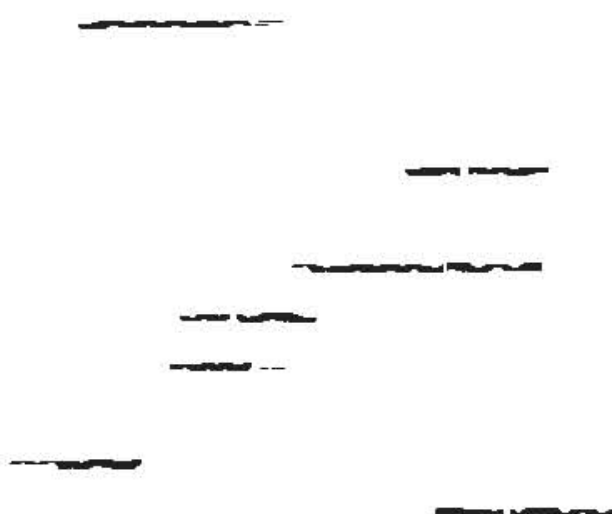

Fig. 43 - I mierosismi con periodi di 8-8,5 sec - associati ai sistemi di onde significative, provocati nelia zona di mare prospiciente le coste occidentali dell'Irlanda dalla perturbazione vasta o profonda, di cui alle figure 40 44, si propagano alle massime distanze. Quello sopra riportato ò un tratto della registrazione ottenuta presso Ia Stazione Sismica di Roma (Citta Universitaria) da un apparecehio Galitzin-Wilip alla dsitanza di oltre $2.000 \mathrm{~km}$. La mancanza di perturbazioni secondarie, di qualehe importanza, nel Tirreno settentrionale, eonsente una registrazione pressoclié indisturbata.

Fig. 43 - The microseisms with periods of $8.8,5 \mathrm{sec}$, associated to signifieant wave systems and raised in the sea area off the West coast of Ireland by the vast and deep perturbation shown by figs. 40 and 44 , propagate to maximum distances. The above is a section of the recording male in the Seismic Station of University City of Rome by a Galitzin-Wlip instrument at over $2000 \mathrm{kms}$ distance. Since no secondary perturbations of a certain weight were present in the Northern Tyrrhenian area, the recording was almost undisturbed. 


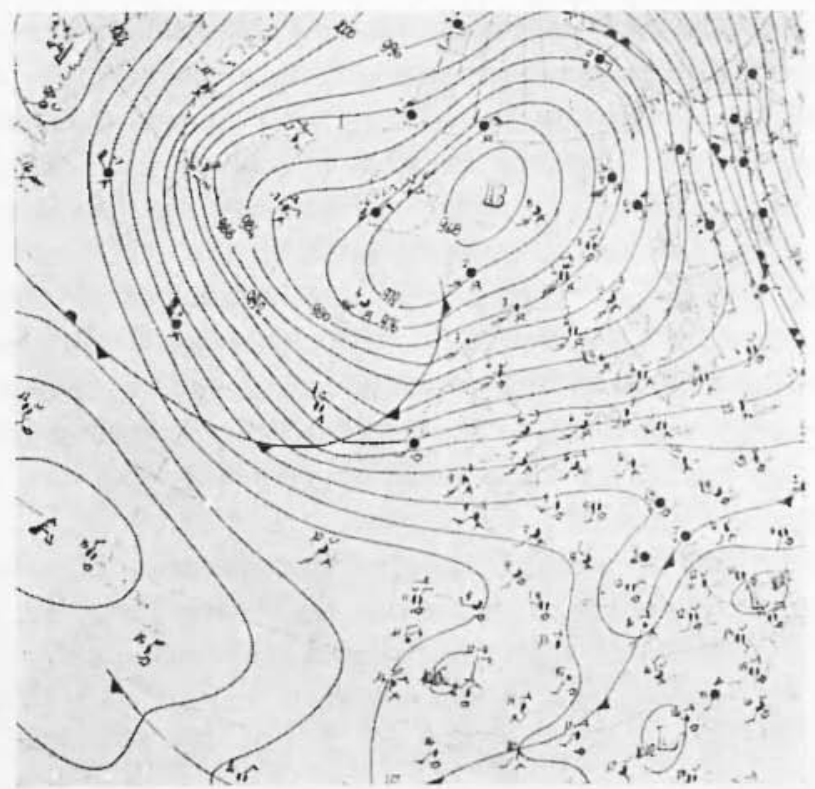

Fig. 44 - Situazione meteorologica sull'Atlantico nord-orientale e sull'Europa, alle ore 12 (G.M.T.) del 14 Febbraio 1971 (Dal "Boll. Meteorol, Quot." del Servizio Meteor. dell'Aer. Mil.).

Fig. 44 - The weather situation for the North-East Atlantic area and for Europe at midday (G.M.T.) of Febr. 14, 1971 (from the Daily Meteorological Bulletin of the Weather Service of the Air Force).

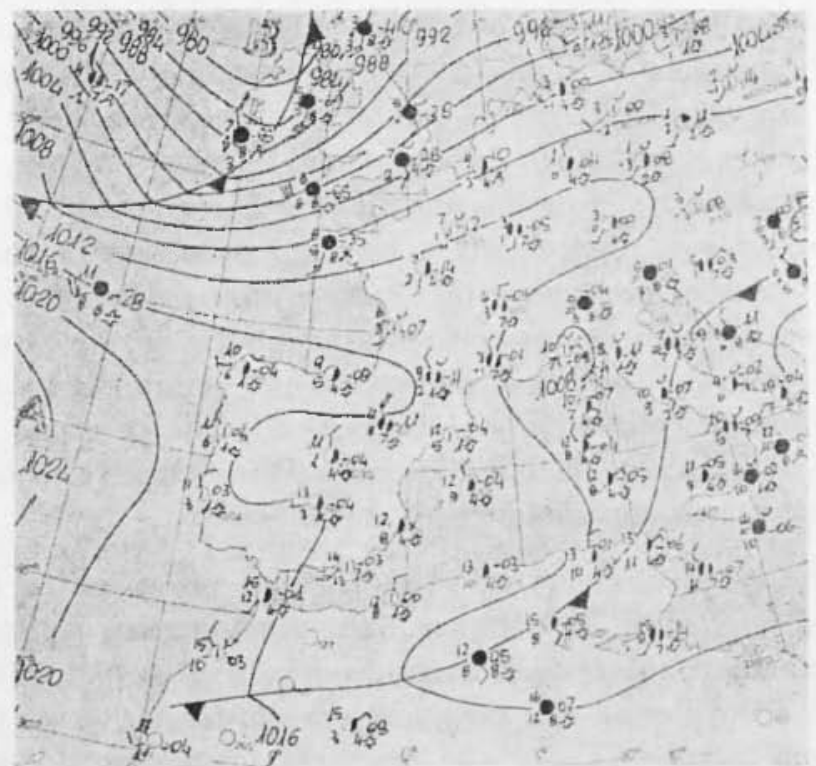

Fig. 45 - Situazione meteorologica sull'Atlantico e sull'Europa alle ore 18 (G.M.T.) del 14 Febbraio 1971.

Fig. 45 - The weather situation for the Atlantic area and for Europe at $6^{\mathrm{h}}$ p.m. (G.M.T.) of lebruary 14, 1971 . 
b) I microsismi originanti da mari interni, localmente possono presentare tutta una gamma di periodi, la quelli con periodi inferiori al secondo, ai periodi massimi, causati dalle coperiodali onde significative, proprie del tratto di mare dove i microsismi hanno origine. Solo questi ultimi risultano persistenti e possono propagarsi a distanze di parechie centinaia di chilometri. Dal golfo di Trieste originano microsismi persistenti, con periodi dell'ordine di $3^{5}$, mentre dal tratto di mare a Nori dell'isola d'Elba insorgono microsismi persistenti i (ui perioli sono di $4^{\text {s- }}$ ô $^{s}$ ca. Stazioni sufficientemente lontane dalle zone-origine, registrano solo microsismi persistenti: poiché i microsismi non subiscono dispersione apprezzabile, dal loro periodo si puó risalire alla zona di provenienza.

I microsismi da mari interni trovano ostacolo, nella loro propagazione, in corrispondenza di sistemi montuosi. P.es., gli Appennini formano una specie di sbarramento alla propagazione dei microsismi del golfo di Venezia, che vengono invece esaltati dalla Val Padana. Anche i microsismi dal Tirreno sono fortemente smorzati dagli Appennini, mentre i microsismi, che originano dal Mar Ligure si propagano nell'alta Val Padana, attraverso la "finestra" interposta fra le Alpi a gli Appennini: fra il Colle di Cadibona e il Passo dei Giovi.

c) Lo stesso meccanismo invocato per spiegare l'insorgere dei microsismi da bacini chinsi o da mari interni, vale anche per le tempeste microsismiche d'origine oceanica: l'interazione fra atmosfera ed idrosfera. Le grandi estensioni oceaniche e la durata delle perturbazioni cicloniche, consentono la formazione di estesi "fetch ", nei quali le onde significative possono raggiungere il loro massimo sviluppo: ciò spiega l'elevato periodo dei microsismi persistenti d'origine oceanica $(\tilde{0}-6)$ secondi per tempeste prossime alle coste scandinave; $7-9$ secondi per le vaste depressioni atlanticlıe dal golfo di Biscaglia, alle isole Britanniche ed oltre). I microsismi più sviluppati insorgono generalmente non lontano dalle coste, per onde che "sentono il fonilo" quando le fluttuazioni della pressione, in fase positiva, interagiscono con le sottostanti coperiodali onde significative.

I microsismi d'origine oceanica possono propagarsi a migliaia e migliaia di chilometri di distanza dalla zona-origine, nell'interno dei continenti, senza subire forti attenuazioni da parte dei sistemi montuosi. Ció è dovuto sia alla maggiore energia trasferita sul fondo nell'interazione aria-acqua, sia alla maggiore lunghezas d'onda, che - 
a differenza di microsismi cla mari interni - consente loro di interessare un più grosso sjessore della crosta terrestre.

Naturalmente, anche nelle zone costicre oceaniche, presso i luoghi d'insorgenza dei microsismi, i sismogrammi presentano generalmente un carattere caotico, per il sovrajporsi di perturbazioni microsismiche associate a sistemi d'onda marina temporanei, sporadici, di periodo inferiore a quello dei microsismi provocati dalle onde signiticative. Solo questi ultimi però permangono al di fuori di una zona di raggio variabile - comunque sempre limitato - e si propagano able massime distanze, conservando il periodo origine.

\section{BIBLIOGRAFIA}

(1) Bossolasco M., Cicconi G., Dagnino I., Eva C., Ta Stazione Sismica dell'Università di Genova. "Geofisica o Heteorologia ", XIII, 1-2, 1964.

(2) Bossolasco M., Canny A., Cicconi G., Eva C., Contributo allo studio dei microsismi. "Geofisica a Meteorologia", XVI, 5-15, 1967.

(a) CaLor P., L'agitazione miorosismica destata dalle perturbazioni meteoro. logiche: I. Hicrosismi da piccoli bacini chiusi. Atti Ace. Naz. Lincei, Renticonti Classe Scienze fis., mat. e nat., L, 5, 1971.

(4) Calor P., I'agitazione mierosismica destata dalle perhurbazioni meleorologiche: II. Origine dei microsismi associati al transito dei cicloni. Atti Ace. Naz. Lincei, Renticonti Classe Scienze fis., mat, e nat. (1971, in corso di stampa). 